\title{
USER'S GUIDE TO VISCOSITY SOLUTIONS OF SECOND ORDER PARTIAL DIFFERENTIAL EQUATIONS
}

\author{
MICHAEL G. CRANDALL, HITOSHI ISHII, AND PIERRE-LOUIS LIONS
}

\begin{abstract}
The notion of viscosity solutions of scalar fully nonlinear partial differential equations of second order provides a framework in which startling comparison and uniqueness theorems, existence theorems, and theorems about continuous dependence may now be proved by very efficient and striking arguments. The range of important applications of these results is enormous. This article is a self-contained exposition of the basic theory of viscosity solutions.
\end{abstract}

\section{INTRODUCTION}

The theory of viscosity solutions applies to certain partial differential equations of the form $F\left(x, u, D u, D^{2} u\right)=0$ where $F: \mathbb{R}^{N} \times \mathbb{R} \times \mathbb{R}^{N} \times \mathscr{S}(N) \rightarrow \mathbb{R}$ and $\mathscr{S}(N)$ is the set of symmetric $N \times N$ matrices. The primary virtues of this theory are that it allows merely continuous functions to be solutions of fully nonlinear equations of second order, that it provides very general existence and uniqueness theorems and that it yields precise formulations of general boundary conditions. Moreover, these features go hand-in-hand with a great flexibility in passing to limits in various settings and relatively simple proofs. In the expression $F\left(x, u, D u, D^{2} u\right) u$ will be a real-valued function defined on some subset $\mathscr{O}$ of $\mathbb{R}^{N}, D u$ corresponds to the gradient of $u$ and $D^{2} u$ corresponds to the matrix of second derivatives of $u$. However, as explained below, $D u$ and $D^{2} u$ will not have classical meanings and, in fact, the examples exhibited in $\S 1$ will convince the reader that the theory encompasses classes of equations that have no solutions that are differentiable in the classical sense.

In order that the theory apply to a given equation $F=0$, we will require $F$ to satisfy a fundamental monotonicity condition

$$
F(x, r, p, X) \leq F(x, s, p, Y) \text { whenever } r \leq s \text { and } Y \leq X ;
$$

where $r, s \in \mathbb{R}, x, p \in \mathbb{R}^{N}, X, Y \in \mathscr{S}(N)$ and $\mathscr{S}(N)$ is equipped with its usual order.

Received by the editors November 16, 1990.

1991 Mathematics Subject Classification. Primary 35D05, 35B50, 35J60, 35K55; Secondary 35B05, 35B25, 35F20, 35J25, 35J70, 35K20, 35K15, 35K65.

Key words and phrases. Viscosity solutions, partial differential equations, fully nonlinear equations, elliptic equations, parabolic equations, Hamilton-Jacobi equations, dynamic programming, nonlinear boundary value problems, generalized solutions, maximum principles, comparison theorems, Perron's method.

First author supported in part by the Army Research Office DAAL03-87-K-0043 and 03-90-G-0102, National Science Foundation DMS-8505531 and 90-02331, and Office of Naval Research N00014-88-K-0134.

This paper was given as a Progress in Mathematics Lecture at the August 8-11, 1990 meeting of the American Mathematical Society in Columbus, Ohio. 
Regarding (0.1) as made up of the two conditions

$$
F(x, r, p, X) \leq F(x, s, p, X) \text { whenever } r \leq s,
$$

and

$$
F(x, r, p, X) \leq F(x, r, p, Y) \text { whenever } Y \leq X,
$$

we will give the name "degenerate ellipticity" to the second. That is, $F$ is said to be degenerate elliptic if (0.3) holds. When (0.2) also holds (equivalently, (0.1) holds), we will say that $F$ is proper.

The examples given in $\S 1$ will illustrate the fact that the antimonotonicity in $X$ is indeed an "ellipticity" condition. The possibility of "degeneracies" is clearly exhibited by considering the case in which $F(x, r, p, X)$ does not depend on $X$-it is then degenerate elliptic. The monotonicity in $r$, while easier to understand, is a slightly subtle selection criterion that, in particular, excludes the use of the viscosity theory for first order equations of the form $b(u) u_{x}=f(x)$ in $\mathbb{R}$ when $b$ is not a constant function, since then $F(x, r, p)=$ $b(r) p-f(x)$ is not nondecreasing in $r$ for all choices of $p$ (scalar conservation laws are outside of the scope of this theory).

The presentation begins with $\S 1$, which, as already mentioned, provides a list of examples. This rather long list is offered to meet several objectives. First, we seek to bring the reader to our conviction that the scope of the theory is quite broad while providing a spectrum of meaningful applications and, at the same time, generating some insight as regards the fundamental structural assumption (0.1). Finally, in the presentation of examples involving famous second order equations, the very act of writing the equations in a form compatible with the theory will induce an interesting modification of the classical viewpoint concerning them.

In $\S 2$ we begin an introductory presentation of the basic facts of the theory. The style is initially leisurely and expository and technicalities are minimized, although complete discussions of various key points are given and some simple arguments inconveniently scattered in the literature are presented. Results are illustrated with simple examples making clear their general nature. Section 2 presents the basic notions of solution used in the theory, the analytical heart of which lies in comparison results. Accordingly, $\S 3$ is devoted to explaining comparison results in the simple setting of the Dirichlet problem; roughly speaking, they are proved by methods involving extensions of the maximum principle to semicontinuous functions. Once these comparison results are established, existence assertions can be established by Perron's method, a rather striking tale that is told in $\S 4$. With this background in hand, the reader will have an almost complete (sub)story and with some effort (but not too much!) should be able to absorb in an efficient way some of the more technical features of the theory that are outlined in the rest of the paper.

Other important ideas are to be found in $\S 6$, which is concerned with the issue of taking limits of viscosity solutions and applications of this and in $\S 7$, which describes the adaptation of the theory to accommodate problems with other boundary conditions and problems in which the boundary condition cannot be strictly satisfied. In the later case, the entire problem has a generalized interpretation for which there is often existence and uniqueness. While the description of these results is deferred to $\S 7$, they are fundamental and dramatic. 
For example, if $G(p, X)$ is uniformly continuous, degenerate elliptic, and independent of $x$ and $\Omega \subset \mathbb{R}^{N}$ is open and bounded, $n(x)$ is the unit exterior normal at the point $x$ in its smooth boundary $\partial \Omega$ and $f \in C(\bar{\Omega})$, then the Neumann problem

$$
u+G\left(D u, D^{2} u\right)-f(x)=0 \quad \text { in } \Omega, \quad u_{n}=0 \quad \text { on } \partial \Omega
$$

has a unique properly interpreted solution (which may not satisfy $u_{n}=0$ on $\partial \Omega$ ).

Sections 5, 8, 9 discuss variations of the basic material and need not be read in sequence. Section 10 is devoted to a commentary about applications (which are not treated in the main text), references, and possible lines of future development of the subject. We conclude with an appendix where the reader will find a self-contained presentation of the proof of the analytical heart on the presentation we have chosen.

References are not given in the main text (with the exception of $\S 10$ ), but are to be found at the end of each section. In particular, the reader should look to the end of a section for further comments, references that contain details ommitted in the main text, and technical generality, historical comments, etc. The references, while numerous, are not intended to be complete, except that we have sought to represent all the major directions of research and areas of application. There are original aspects of the current presentation and the reader will note differences between the flavor and clarity of our presentation and that of many of the citations. However, equipped with the view presented here, we hope and expect that perusing the amazing literature that has so quickly matured will be a much more rewarding and efficient endeavor.

We are grateful to R. Dorroh, M. Kocan and A. Swiech for their kind help in reducing the number of errors herein.

\section{Contents}

\section{Examples}

2. The notion of viscosity solutions

3. The maximum principle for semicontinuous functions and comparison for the Dirichlet problem

4. Perron's method and existence

5. Comparison: Variations on the theme

5.A. Comparison with more regularity

5.B. Estimates from comparison

5.C. Comparison with strict inequalities and without coercivity in $u$

5.D. Comparison and existence of unbounded solutions on unbounded domains

6. Limits of viscosity solutions and an application

7. General and generalized boundary conditions

7.A. Boundary conditions in the viscosity sense 
7.B. Existence and uniqueness for the Neumann problem

7.C. The generalized Dirichlet problem

7. $C^{\prime}$. The state constraints problem

7.D. A remark on (BC) in the classical sense

7.E. Fully nonlinear boundary conditions

8. Parabolic problems

9. Singular equations: An example from geometry

10. Applications and perspectives

APPENDIX The proof of Theorem 3.2

\section{EXAMPLES}

We will record here many examples of degenerate elliptic equations mentioning, when appropriate, areas in which they arise. The reader is invited to scan the list and pause where interested-it is possible to proceed to $\S 2$ at any stage. Below we will say either that a function $F(x, r, p, X)$ is degenerate elliptic or that the equation $F\left(x, u, D u, D^{2} u\right)=0$ is degenerate elliptic or that the "operator" or expression $F\left(x, u, D u, D^{2} u\right)$ is degenerate elliptic and always mean the same thing, i.e., (0.3) holds; the term "proper" is used in a similar fashion.

Example 1.1. Laplace's equation. We revisit an old friend, the equation

$$
-\Delta u+c(x) u=f(x)
$$

-note the sign in front of the Laplacian. The corresponding $F$ is naturally given by $F(x, r, p, X)=-\operatorname{trace}(X)+c(x) r-f(x)$, which is proper if $c \geq 0$.

Example 1.2. Degenerate elliptic linear equations. Example 1.1 immediately extends to the more general linear equation

$$
-\sum_{i, j=1}^{N} a_{i, j}(x) \frac{\partial^{2} u}{\partial x_{i} \partial x_{j}}+\sum_{i=1}^{N} b_{i}(x) \frac{\partial u}{\partial x_{i}}+c(x) u(x)=f(x)
$$

where the matrix $A(x)=\left\{a_{i, j}(x)\right\}$ is symmetric; the corresponding $F$ is

$$
F(x, r, p, X)=-\operatorname{trace}(A(x) X)+\sum_{i=1}^{N} b_{i}(x) p_{i}+c(x) r-f(x)
$$

In this case, $F$ is degenerate elliptic if and only if $A(x) \geq 0$ and it is proper if also $c(x) \geq 0$. In the event that there are constants $\lambda, \Lambda>0$ such that $\lambda I \leq A(x) \leq \Lambda I$ for all $x$ where $I$ is the identity matrix, $F$ is said to be uniformly elliptic.

Of course, the linear equation in divergence form

$$
-\sum_{i, j=1}^{N} \frac{\partial}{\partial x_{i}}\left(a_{i, j}(x) \frac{\partial u}{\partial x_{j}}\right)+\sum_{j=1}^{N} b_{j}(x) \frac{\partial u}{\partial x_{i}}+c(x) u(x)=f(x)
$$

can be written as above with

$$
F(x, r, p, X)=-\operatorname{trace}(A(x) X)+\sum_{j=1}^{N}\left(b_{j}(x)-\sum_{i=1}^{N} \frac{\partial a_{i, j}}{\partial x_{i}}(x)\right) p_{j}+c(x) r-f(x)
$$


provided that the indicated derivatives of the $a_{i, j}$ exist.

We leave the interesting class of linear equations to turn to the totally degenerate case of first order equations.

Example 1.3. First order equations. The main point is that a first order operator $F(x, u, D u)$ is always degenerate elliptic and thus it is proper if and only if $F(x, r, p)$ is nondecreasing in $r \in \mathbb{R}$. Proper equations of the form $F(x, u, D u)=0$ play a fundamental role in the classical Calculus of Variations and in Optimal Control Theory of ordinary differential equations; in this context they are often called Bellman or Hamilton-Jacobi equations and then $F(x, r, p)$ is convex in $(r, p)$. These equations, in the full generality of nearly arbitrary proper functions $F$, are also crucial in Differential Games Theory where they are known as Isaacs's equations.

Example 1.4. Quasilinear elliptic equations in divergence form. The usual notion of ellipticity for equations of the form

$$
-\sum_{i=1}^{N} \frac{\partial}{\partial x_{i}}\left(a_{i}(x, D u)\right)+b(x, u, D u)=0
$$

is the monotonicity of the vector field $a(x, p)$ in $p$ as a mapping from $\mathbb{R}^{N}$ to $\mathbb{R}^{N}$. If enough regularity is available to carry out the differentiation, we write $(1.4)$ as

$$
-\sum_{i, j=1}^{N} \frac{\partial a_{i}}{\partial p_{j}}(x, D u) \frac{\partial^{2} u}{\partial x_{i} \partial x_{j}}+b(x, u, D u)-\sum_{i=1}^{N} \frac{\partial a_{i}}{\partial x_{i}}(x, D u)=0
$$

and correspondingly set

$$
F(x, r, p, X)=-\operatorname{trace}\left(\left(D_{p} a(x, p)\right) X\right)+b(x, r, p)-\sum_{i=1}^{N} \frac{\partial a_{i}}{\partial x_{i}}(x, p) .
$$

The monotonicity of $a$ in $p$ is precisely the condition that guarantees that $F$ is degenerate elliptic, and then it is proper provided we ask that $b$ be nondecreasing in $r$.

Two well-known instances are provided by the equation of minimal surfaces in nonparametric form and the " $m$-Laplace's" equation that are given, respectively, by $a(x, p)=\left(1+|p|^{2}\right)^{-1 / 2} p, b=b(x, u)$ and $a(x, p)=|p|^{m-2} p, b=$ $b(x, r)$ where $m \in(1, \infty)$. Computations show that the corresponding $F$ 's are, respectively,

$F(x, r, p, X)=-\left(1+|p|^{2}\right)^{-\frac{1}{2}} \operatorname{trace}(X)+\left(1+|p|^{2}\right)^{-\frac{3}{2}} \operatorname{trace}((p \otimes p) X)+b(x, r)$ and

$$
F(x, r, p, X)=-|p|^{m-2} \operatorname{trace}(X)-(m-2)|p|^{m-4} \operatorname{trace}((p \otimes p) X)+b(x, r) .
$$

Example 1.5. Quasilinear elliptic equations in nondivergence form. The equation

$$
-\sum_{i, j=1}^{N} a_{i, j}(x, p) \frac{\partial^{2} u}{\partial x_{i} \partial x_{j}}+b(x, u, D u)=0
$$


where $A(x, p)=\left\{a_{i, j}(x, p)\right\} \in \mathscr{S}(N)$, contains all of the above as special cases and corresponds to

$$
F(x, r, p, X)=-\operatorname{trace}(A(x, p) X)+b(x, r, p),
$$

which is proper if $A \geq 0$ and $b$ is nondecreasing with respect to $r$. Two relevant special cases are

$$
-\nu \Delta u+f(x, u, D u)=0
$$

with $\nu>0$ and $f$ nondecreasing in $u$, which may be regarded as a firstorder Hamilton-Jacobi equation perturbed by an additional "viscosity" term $-\nu \Delta u$ (equations of this type arise in optimal stochastic control), and the Lévi's equation

$$
\begin{aligned}
& -\left(\frac{\partial^{2} u}{\partial x_{1}^{2}}+\frac{\partial^{2} u}{\partial x_{2}^{2}}\right)\left(1+\left(\frac{\partial u}{\partial x_{3}}\right)^{2}\right)-\frac{\partial^{2} u}{\partial x_{3}^{2}}\left(\left(\frac{\partial u}{\partial x_{1}}\right)^{2}+\left(\frac{\partial u}{\partial x_{2}}\right)^{2}\right) \\
& +2 \frac{\partial^{2} u}{\partial x_{1} \partial x_{3}}\left(\frac{\partial u}{\partial x_{3}} \frac{\partial u}{\partial x_{1}}-\frac{\partial u}{\partial x_{2}}\right)+2 \frac{\partial^{2} u}{\partial x_{2} \partial x_{3}}\left(\frac{\partial u}{\partial x_{3}} \frac{\partial u}{\partial x_{2}}+\frac{\partial u}{\partial x_{1}}\right)=0,
\end{aligned}
$$

which is the nonparametric formulation for a hypersurface in $\mathbb{C}^{2}$ with vanishing Lévi's form. Note that in this example $F=-\operatorname{trace}(A(p) X)$ where

$$
A(p)=\left(\begin{array}{ccc}
1+p_{3}^{2} & 0 & p_{3} p_{1}-p_{2} \\
0 & 1+p_{3}^{2} & p_{3} p_{2}+p_{1} \\
p_{3} p_{1}-p_{2} & p_{3} p_{2}+p_{1} & p_{1}^{2}+p_{2}^{2}
\end{array}\right)
$$

so that $A \geq 0$ but $\operatorname{det}(A(p))=0$ for all $p$.

Example 1.6. Hamilton-Jacobi-Bellman and Isaacs equations. Hamilton-JacobiBellman and Isaacs equations are, respectively, the fundamental partial differential equations for stochastic control and stochastic differential games. The natural setting involves a collection of elliptic operators of second-order depending either on one parameter $\alpha$ (in the Hamilton-Jacobi-Bellman case) or two parameters $\alpha, \beta$ (in the case of Isaacs's equations). These parameters lie in index sets we will not display in the discussion. Thus we take as ingredients proper expressions of the form

$$
\mathscr{L}^{\alpha} u=-\sum_{i, j=1}^{N} a_{i, j}^{\alpha}(x) \frac{\partial^{2} u}{\partial x_{i} \partial x_{j}}+\sum_{i=1}^{N} b_{i}^{\alpha}(x) \frac{\partial u}{\partial x_{i}}+c^{\alpha}(x) u(x)-f^{\alpha}(x)
$$

or

$$
\mathscr{L}^{\alpha, \beta} u=-\sum_{i, j=1}^{N} a_{i, j}^{\alpha, \beta}(x) \frac{\partial^{2} u}{\partial x_{i} \partial x_{j}}+\sum_{i=1}^{N} b_{i}^{\alpha, \beta}(x) \frac{\partial u}{\partial x_{i}}+c^{\alpha, \beta}(x) u(x)-f^{\alpha, \beta}(x)
$$

where all the coefficients are bounded with respect to $\alpha$ or $\alpha, \beta$. HamiltonJacobi-Bellman equations include those of the form

$$
\sup _{\alpha}\left\{\mathscr{L}^{\alpha} u\right\}=0
$$

while

$$
\sup _{\alpha} \inf _{\beta}\left\{\mathscr{L}^{\alpha, \beta} u\right\}=0
$$


is a typical Isaacs's equation. The corresponding nonlinearities $F$ have the form

$$
F(x, r, p, X)=\sup _{\alpha}\left[-\operatorname{trace}\left(A^{\alpha}(x) X\right)+\left\langle b^{\alpha}(x), p\right\rangle+c^{\alpha}(x) r-f^{\alpha}(x)\right]
$$

and

$$
\begin{aligned}
F(x, r, p, X)=\sup _{\alpha} \inf _{\beta}[ & -\operatorname{trace}\left(A^{\alpha, \beta}(x) X\right) \\
& \left.+\left\langle b^{\alpha, \beta}(x), p\right\rangle+c^{\alpha, \beta}(x) r-f^{\alpha, \beta}(x)\right],
\end{aligned}
$$

each of which is clearly also proper. Notice that in the first case $F$ is convex in $(r, p, X)$ while in the second case this is not so; indeed, if one allows for "unbounded envelopes" (i.e., coefficients that are unbounded in the parameters), one can show that essentially any proper $F$ satisfying minor regularity assumptions can be represented as a "sup inf" of linear expressions as above.

Indeed, the above process is quite general and does not require linear ingredients. Suppose $F^{\alpha, \beta}$ is proper for each $\alpha, \beta$. Then $F(x, r, p, X)=$ $\sup _{\alpha} \inf _{\beta} F^{\alpha, \beta}(x, r, p, X)$ and $F(x, r, p, X)=\inf _{\alpha} \sup _{\beta} F^{\alpha, \beta}(x, r, p, X)$ are also evidently proper (for the moment, we set aside considerations of finiteness and continuity).

Example 1.7. Obstacle and gradient constraint problems. A special case of the last remarks above is met in the consideration of "obstacle problems." Very general forms of such problems may be written

$$
\max \left\{F\left(x, u, D u, D^{2} u\right), u-f(x)\right\}=0
$$

or

$$
\min \left\{F\left(x, u, D u, D^{2} u\right), u-f(x)\right\}=0
$$

or even

$$
\max \left\{\min \left\{F\left(x, u, D u, D^{2} u\right), u-f(x)\right\}, u-g(x)\right\}=0 .
$$

In accordance with remarks made in the previous example, if $F$ is proper then so are (1.12)-(1.14).

Likewise, "gradient constraints" may be imposed in this way. A typical example corresponds to $\max \left\{F\left(x, u, D u, D^{2} u\right),|D u|-g(x)\right\}=0$.

Example 1.8. Functions of the eigenvalues. For $X \in \mathscr{S}(N)$ we let $\lambda_{1}(X), \ldots$, $\lambda_{N}(X)$ be its eigenvalues arranged in increasing order, $\lambda_{i}(X) \leq \lambda_{i+1}(X)$. If $g\left(x, r, p, s_{1}, \ldots, s_{N}\right)$ is defined on $\mathbb{R}^{3 N+1}$ and is nondecreasing in $r$ and each $s_{i}$, then $F(x, r, p, X)=g\left(x, r, p,-\lambda_{1}(X), \ldots,-\lambda_{N}(X)\right)$ is proper. For instance, $F(X)=-\max \left\{\lambda_{1}(X), \ldots, \lambda_{N}(X)\right\}=-\lambda_{N}(X), F(X)=-\min \left\{\lambda_{1}(X)\right.$, $\left.\ldots, \lambda_{N}(X)\right\}=-\lambda_{1}(X)$ and $F(X)=-\left(\lambda_{2}(X)\right)^{3}$ are degenerate elliptic. Another example is

$$
F(x, r, p, X)=-|\operatorname{trace}(X)|^{m-1} \operatorname{trace}(X)+|p|^{q}+c(x) r-f(x)
$$

where $c \geq 0$ and $m, q>0$. The corresponding equation is

$$
-|\Delta u|^{m-1} \Delta u+|D u|^{q}+c(x) u=f(x),
$$

which provides another example of the generality we are dealing with, even if we have no interpretation of this equation in mind. 
Example 1.9. Sums and increasing functions of proper functions. If $F_{i}$ is proper for $i=1, \ldots, M$, then so is $F_{1}+\cdots+F_{M}$. More generally, if $g\left(s_{1}, \ldots, s_{M}\right)$ is nondecreasing in each variable, then $g\left(F_{1}, \ldots, F_{M}\right)$ is proper. One may build very complex examples using the cases discussed above and these remarks.

Example 1.10. Parabolic problems. We just observe that if $(x, r, p, X) \rightarrow$ $F(t, x, r, p, X)$ is proper for fixed $t \in[0, T]$, then so is the associated "parabolic" problem

$$
u_{t}+F\left(t, x, u, D u, D^{2} u\right)=0
$$

when considered as an equation in the $N+1$ independent variables $(t, x)$. We mention only one example (there are, of course, infinitely many) that has some geometrical interest since it describes the evolution of a surface (given by a level set of the initial condition) with a motion along its normal with a speed proportional to the mean curvature

$$
u_{t}-|D u| \operatorname{div}\left(\frac{D u}{|D u|}\right)=0 .
$$

Carrying out the differentiations yields

$$
u_{t}-\Delta u+\sum_{i, j=1}^{N} \frac{\partial^{2} u}{\partial x_{i} \partial x_{j}} \frac{\partial u}{\partial x_{i}} \frac{\partial u}{\partial x_{j}}|D u|^{-2}=0 .
$$

This may be written in the form (1.15) with

$$
F(x, p, X)=-\operatorname{trace}\left(\left(I-\frac{p \otimes p}{|p|^{2}}\right) X\right) \text {. }
$$

Example 1.11. Monge Ampère equations. The Monge-Ampère equation may be written as

$$
u \text { is convex, } \quad \operatorname{det}\left(D^{2} u\right)=f(x, u, D u)
$$

where $f(x, r, p) \geq 0$. We are dealing here with the real Monge-Ampère equation, but everything that will be said adapts to the complex case and to other curvature equations. Allowing $F$ to be discontinuous (even more, to become infinite), we may write (1.18) in our form by putting

$$
F(x, r, p, X)= \begin{cases}-\operatorname{det}(X)+f(x, r, p) & \text { if } X \geq 0 \\ +\infty & \text { otherwise }\end{cases}
$$

$F$ is then degenerate elliptic. This follows from the fact that

$$
g\left(s_{1}, \cdots, s_{N}\right)= \begin{cases}\prod_{i=1}^{N} s_{i} & \text { if } s_{i} \geq 0, i=1, \ldots, N, \\ -\infty & \text { otherwise, }\end{cases}
$$

is nondecreasing in each of its arguments and Example 1.8.

Example 1.12. Uniformly elliptic functions. This "example" is really a definition. One says that $F(x, r, p, X)$ is uniformly elliptic if there are constants $\lambda, \Lambda>0$ for which

$$
\lambda \operatorname{trace}(P) \leq F(x, r, p, X-P)-F(x, r, p, X) \leq \Lambda \operatorname{trace}(P) \quad \text { for } P \geq 0
$$


and all $x, r, p, X$ and then calls the constants $\lambda, \Lambda$ ellipticity constants. We note that $L$ in Example 1.2 is uniformly elliptic with constants $\lambda, \Lambda$ exactly when $\lambda \leq \lambda_{1}(A(x))$ and $\lambda_{N}(A(x)) \leq \Lambda$. One notes that sums of uniformly elliptic functions are again uniformly elliptic and that the sup inf process over a family of uniformly elliptic functions with common ellipticity constants produces another such function.

Notes on $§ 1$. We will disappoint the reader in the following sections by not applying the theory developed therein to the many examples given above. The goal of this section was to exhibit clearly the breadth and importance of the class of proper equations. We simply do not have enough space here to develop applications of the theory of these equations beyond that which follows immediately from the general results presented.

Most of the examples listed have been considered via classical approaches. We give some references containing classical presentations: D. Gilbarg and N. S. Trudinger [81] is a basic source concerning linear and quasi-linear uniformly elliptic equations; O. A. Oleinnik and E. V. Radkevic [139], J. J. Kohn and L. Nirenberg [106], and A. V. Ivanov [98] treat degenerate elliptic equations; W. H. Fleming and R. Rishel [75], P. L. Lions [117], and N. V. Krylov [108, 109] are sources for Hamilton-Jacobi-Bellman equations; S. Benton [36] and P. L. Lions [116] discuss first-order Hamilton-Jacobi equations. Most of these references present some of the ways these equations arise.

\section{THE NOTION OF VISCOSITY SOLUTIONS}

It is always assumed that $F$ satisfies (0.1) (i.e., $F$ is proper) and, unless otherwise said, is continuous. To motivate the notions, we begin by supposing that $u$ is $C^{2}$ (i.e., twice continuously differentiable) on $\mathbb{R}^{N}$ and

$$
F\left(x, u(x), D u(x), D^{2} u(x)\right) \leq 0
$$

holds for all $x$ (that is, $u$ is a classical subsolution of $F=0$ or, equivalently, a classical solution of $F \leq 0$ in $\mathbb{R}^{N}$ ). Suppose that $\varphi$ is also $C^{2}$ and $\hat{x}$ is a local maximum of $u-\varphi$. Then calculus implies $D u(\hat{x})=D \varphi(\hat{x})$ and $D^{2} u(\hat{x}) \leq D^{2} \varphi(\hat{x})$ and so, by degenerate ellipticity,

$$
F\left(\hat{x}, u(\hat{x}), D \varphi(\hat{x}), D^{2} \varphi(\hat{x})\right) \leq F\left(\hat{x}, u(\hat{x}), D u(\hat{x}), D^{2} u(\hat{x})\right) \leq 0 .
$$

The extremes of this inequality do not depend on the derivatives of $u$ and so we may consider defining an arbitrary function $u$ to be (some kind of generalized) subsolution of $F=0$ if

$$
F\left(\hat{x}, u(\hat{x}), D \varphi(\hat{x}), D^{2} \varphi(\hat{x})\right) \leq 0
$$

whenever $\varphi$ is $C^{2}$ and $\hat{x}$ is a local maximum of $u-\varphi$. Before making any formal definitions, let us also note that $u(x) \leq u(\hat{x})-\varphi(\hat{x})+\varphi(x)$ for $x$ near $\hat{x}, \varphi \in C^{2}$ and Taylor approximation imply

$$
u(x) \leq u(\hat{x})+\langle p, x-\hat{x}\rangle+\frac{1}{2}\langle X(x-\hat{x}), x-\hat{x}\rangle+o\left(|x-\hat{x}|^{2}\right) \text { as } x \rightarrow \hat{x}
$$

where $p=D \varphi(\hat{x})$ and $X=D^{2} \varphi(\hat{x})$. Moreover, if (2.3) holds for some $(p, X) \in \mathbb{R}^{N} \times \mathscr{S}(N)$ and $u$ is twice differentiable at $\hat{x}$, then $p=D u(\hat{x})$ and $D^{2} u(\hat{x}) \leq X$. Thus if $u$ is a classical solution of $F \leq 0$ it follows that $F(\hat{x}, u(\hat{x}), p, X) \leq 0$ whenever (2.3) holds; we may also consider basing a 
definition of nondifferentiable solutions $u$ of $F \leq 0$ on this fact. Roughly speaking, pursuing (2.2) leads to notions based upon test functions $\varphi$ but does not immediately lead us, as will pursuing $(2.3)$, to define " $\left(D u, D^{2} u\right)$ " for nondifferentiable functions $u$, which will turn out to be a good idea. For this reason, we begin by developing the line suggested by (2.3). Next we introduce a set $\mathscr{O} \subset \mathbb{R}^{N}$ on which $F \leq 0$ is to hold and the appropriate notation to deal with inequalities like (2.3) "on $\mathscr{O}$." At the moment, $\mathscr{O}$ is arbitrary; later we require it to be locally compact. Taking off from (2.3), if $u: \mathscr{O} \rightarrow \mathbb{R}, \hat{x} \in \mathscr{O}$, and (2.3) holds as $\mathscr{O} \ni x \rightarrow \hat{x}$, we say $(p, X) \in J_{\mathscr{\theta}}^{2,+} u(\hat{x})$ (the second-order "superjet" of $u$ at $\hat{x}$ ). This defines a mapping $J_{\mathscr{O}}^{2,+} u$ from $\mathscr{O}$ to the subsets of $\mathbb{R}^{N} \times \mathscr{S}(N)$.

Example 2.1. By way of illustration, if $u$ is defined on $\mathbb{R}$ by

$$
u(x)= \begin{cases}0 & \text { for } x \leq 0, \\ a x+\frac{b}{2} x^{2} & \text { for } x \geq 0,\end{cases}
$$

then $J_{[-1,0]}^{2,+} u(0)=((-\infty, 0) \times \mathbb{R}) \cup(\{0\} \times[0, \infty))$, while

$$
J_{\mathbb{R}}^{2,+} u(0)= \begin{cases}\varnothing & \text { if } a>0, \\ \{0\} \times[\max \{0, b\}, \infty) & \text { if } a=0, \\ ((a, 0) \times \mathbb{R}) \cup(\{0\} \times[0, \infty)) \cup(\{a\} \times[b, \infty)) & \text { if } a<0\end{cases}
$$

Having thought through this example, the reader will see that $J_{\mathscr{\theta}}^{2,+} u(x)$ depends on $\mathscr{O}$ but realize it is the same for all sets $\mathscr{O}$ for which $x$ is an interior point; we let $J^{2}+u(x)$ denote this common value. If we repeat the above discussion after switching the inequality sign in (2.3), we arrive at the definitions of the second-order "subjets" $J_{\mathscr{Q}}^{2,-} u, J^{2,-} u$; equivalently, $J_{\mathscr{O}}^{2,-} u(x)=$ $-J_{\mathscr{\theta}}^{2,+}(-u)(x)$, etc.

We are ready to define the notions of viscosity subsolutions, supersolutions, and solutions. It will be useful to have the notations

$$
\begin{aligned}
& \operatorname{USC}(\mathscr{O})=\{\text { upper semicontinuous functions } u: \mathscr{O} \rightarrow \mathbb{R}\}, \\
& \operatorname{LSC}(\mathscr{O})=\{\text { lower semicontinuous functions } u: \mathscr{O} \rightarrow \mathbb{R}\} .
\end{aligned}
$$

Definition 2.2. Let $F$ satisfy $(0.1)$ and $\mathscr{O} \subset \mathbb{R}^{N}$. A viscosity subsolution of $F=0$ (equivalently, a viscosity solution of $F \leq 0$ ) on $\mathscr{O}$ is a function $u \in$ $\operatorname{USC}(\mathscr{O})$ such that

$$
F(x, u(x), p, X) \leq 0 \text { for all } x \in \mathscr{O} \text { and }(p, X) \in J_{\mathscr{O}}^{2,+} u(x) .
$$

Similarly, a viscosity supersolution of $F=0$ on $\mathscr{O}$ is a function $u \in \operatorname{LSC}(\mathscr{O})$ such that

$$
F(x, u(x), p, X) \geq 0 \text { for all } x \in \mathscr{O} \text { and }(p, X) \in J_{\mathscr{O}}^{2,-} u(x) .
$$

Finally, $u$ is a viscosity solution of $F=0$ in $\mathscr{O}$ if it is both a viscosity subsolution and a viscosity supersolution of $F=0$ in $\mathscr{O}$.

Remarks 2.3. Since these "viscosity notions" are the primary ones for the current discussion, we immediately agree (at least, we hope you agree) to drop the term "viscosity" and hereafter simply refer to subsolutions, supersolutions, and solutions. This is a happy idea, as the term "viscosity," which lacks elegance, is 
an artifact of the origin of this theory in the study of first-order equations and the name was then motivated by the consistency of the notion with the method of "vanishing viscosity," which is irrelevant for many second-order equations. It follows from the discussion preceding the definition that, for example, if $u$ is a solution of $F \leq 0$ in $\mathscr{O}, \varphi$ is $C^{2}$ in a neighborhood of $\mathscr{O}$, and $u-\varphi$ has a local maximum (relative to $\mathscr{O}$ ) at $\hat{x} \in \mathscr{O}$, then (2.2) holds. Analogous remarks hold for supersolutions. These remarks motivate the requirement that a subsolution be upper semicontinuous, etc., in the sense that producing maxima of upper semicontinous functions is straightforward. Solutions, being both upper semicontinuous and lower semicontinuous, are continuous. One might ask if the validity of (2.2) for all $\varphi \in C^{2}$ (with the maxima relative to $\mathscr{O}$ ) for an upper semicontinuous function $u$ is equivalent to $u$ being a subsolution. This is so. In fact, if $\hat{x} \in \mathscr{O}$ then $J_{\mathscr{O}}^{2,+} u(\hat{x})=\left\{\left(D \varphi(\hat{x}), D^{2} \varphi(\hat{x})\right): \varphi\right.$ is $C^{2}$ and $u-\varphi$ has a local maximum at $\left.\hat{x}\right\}$; we leave the proof as an interesting exercise.

We next record the definitions of the closures of the set-valued mappings needed in the next section.

With the above notation, for $x \in \mathscr{O}$, we set

$$
\begin{aligned}
\bar{J}_{\mathscr{O}}^{2,+} u(x)=\{ & (p, X) \in \mathbb{R}^{N} \times \mathscr{S}(N): \exists\left(x_{n}, p_{n}, X_{n}\right) \in \mathscr{O} \times \mathbb{R}^{N} \times \mathscr{S}(N) \ni \\
& \left.\left(p_{n}, X_{n}\right) \in J_{\mathscr{O}}^{2,+} u\left(x_{n}\right) \text { and }\left(x_{n}, u\left(x_{n}\right), p_{n}, X_{n}\right) \rightarrow(x, u(x), p, X)\right\}
\end{aligned}
$$

and

$$
\begin{aligned}
\bar{J}_{\mathscr{O}}^{2,-} u(x)=\{ & (p, X) \in \mathbb{R}^{N} \times \mathscr{S}(N): \exists\left(x_{n}, p_{n}, X_{n}\right) \in \mathscr{O} \times \mathbb{R}^{N} \times \mathscr{S}(N) \ni \\
& \left.\left(p_{n}, X_{n}\right) \in J_{\mathscr{\theta}}^{2,-} u\left(x_{n}\right) \text { and }\left(x_{n}, u\left(x_{n}\right), p_{n}, X_{n}\right) \rightarrow(x, u(x), p, X)\right\} ;
\end{aligned}
$$

we are abusing standard practice as regards defining closures of set-valued mappings a bit in that we put the extra condition $u\left(x_{n}\right) \rightarrow u(x)$ in the definitions while the graphs of the multifunctions $J_{\mathscr{\theta}}^{2,+} u, J_{\mathscr{\theta}}^{2,-} u$ do not themselves record the values of $u$. The reader may note the use of expressions like " $x_{n} \rightarrow x$ " as an abbreviation for "the sequence $x_{n}$ satisfies $\lim _{n \rightarrow \infty} x_{n}=x$," etc. If $x \in$ interior $(\mathscr{O})$, we define $\bar{J}^{2,+} u(x), \bar{J}^{2,-} u(x)$ in the obvious way.

Remark 2.4. If $u$ is a solution of $F \leq 0$ on $\mathscr{O}$, then $F(x, u(x), p, X) \leq 0$ for $x \in \mathscr{O}$ and $(p, X) \in J_{\mathscr{O}}^{2,+} u(x)$. This remains true, for reasons of continuity, if $(p, X) \in \bar{J}_{\mathscr{\theta}}^{2,+} u(x)$ and $F$ is continuous (or even lower semicontinuous). Similar remarks apply to supersolutions and solutions.

Advice. We advise the reader to either skip the following material or to scan it lightly at the present time and proceed directly to the next section. The comments collected below can be referred to as needed.

Remark 2.5. While the definitions above may seem reasonable, they contain subtleties. In particular, they do not define "operators" on domains in a familiar way. For example, let $N=1$ and consider the equation $\left(u^{\prime}\right)^{2}-1=0$. The functions $|x|$ and $-|x|$ both satisfy this equation classically on $\mathbb{R} \backslash\{0\}$ 
However, the semijets of the function $u(x)=-|x|$ are given by

$$
J^{2,+} u(x)= \begin{cases}\{(-1, X): X \geq 0\} & \text { if } x>0, \\ ((-1,1) \times \mathbb{R}) \cup(\{-1,1\} \times[0, \infty)) & \text { if } x=0, \\ \{(1, X): X \geq 0\} & \text { if } x<0\end{cases}
$$

and

$$
J^{2,-} u(x)= \begin{cases}\{(-1, X): X \leq 0\} & \text { if } x>0 \\ \varnothing & \text { if } x=0 \\ \{(1, X): X \leq 0\} & \text { if } x<0\end{cases}
$$

so $-|x|$ is a solution of the equation, while if $v(x)=|x|$ then $(0,0) \in$ $J^{2,-} v(0)$ and $v$ is not a solution. Similarly, one sees that $v$ does solve $-\left(v^{\prime}\right)^{2}+1=0$. Hence a solution of $F=0$ need not be a solution of $-F=0$ (of course, if $-F$ is not proper, as is typical when $F$ depends on $u, X$, this is meaningless). It is also unknown if the information $H, u, f, g \in C\left(\mathbb{R}^{N}\right)$ and $u$ is a solution of $H(D u)-f(x)=0$ as well as $H(D u)-g(x)=0$ in $\mathbb{R}^{N}$ implies $f=g$ (although it is known that $f=g$ if either $H$ is uniformly continuous or $N=1$ ).

We do not mention these things to disturb the reader; indeed, they will not explicitly appear in the theory. Our purpose is to emphasize that the theory to follow is not a variant of more classical developments.

Remark 2.6. If we consider one of the simplest examples of a discontinuous upper semicontinuous function, namely, the function given by $u(x)=0$ for $x \neq 0, u(0)=1$, we have $J^{2,+} u(0)=\mathbb{R} \times \mathbb{R}$. The relation $F(0,1, p, X) \leq 0$ for $(p, X) \in J^{2,+} u(0)$ is then very restrictive. In fact, a primary role played by allowing semicontinuous subsolutions and supersolutons in the basic theory is only as a technical device to produce continuous solutions via Perron's method as described in $\S 4$. If solutions are produced by other methods (e.g., via numerical or other approximations) there may be no need to invoke results on semicontinuous functions. However, one of the simplest ways to show uniform convergence of approximation schemes, involves comparison of semicontinuous subsolutions and supersolutions. Thus one often deals with semicontinuity mainly as a device in proofs-as such, it is a powerful labor saving device.

Remarks 2.7. We point out some facts concerning second-order semijets.

(i) It is clear that $J_{\mathscr{Q}}^{2,+} u(x)$ is a convex subset of $\mathbb{R}^{N} \times \mathscr{S}(N)$; Example 2.1 shows that it is not necessarily closed. However, a little thought shows that if $p \in \mathbb{R}^{N}$, then $\left\{X:(p, X) \in J_{\mathscr{Q}}^{2,+} u(x)\right\}$ is closed.

(ii) If $\varphi \in C^{2}$ in some neighborhood of $\mathscr{O}$, then $J_{\mathscr{O}}^{2,+}(u-\varphi)(x)=\{(p-$ $\left.\left.D \varphi(x), X-D^{2} \varphi(x)\right):(p, X) \in J_{\mathscr{O}}^{2,+} u(x)\right\}$. As a consequence, the same statement holds if $J_{\mathscr{Q}}^{2,+}$ is replaced everywhere by $J_{\mathscr{Q}}^{2,-}, \bar{J}_{\mathscr{Q}}^{2,+}$, or $\bar{J}_{\mathscr{\theta}}^{2,-}$. Indeed, if $(q, Y) \in J_{\mathscr{\theta}}^{2,+}(u-\varphi)(\hat{x})$, then

$u(x)-\varphi(x) \leq u(\hat{x})-\varphi(\hat{x})+\langle q, x-\hat{x}\rangle+\frac{1}{2}\langle Y(x-\hat{x}), x-\hat{x}\rangle+o\left(|x-\hat{x}|^{2}\right) \quad$ as $x \rightarrow \hat{x}$

and

$$
\varphi(x)-\varphi(\hat{x})=\langle D \varphi(\hat{x}),(x-\hat{x})\rangle+\frac{1}{2}\left\langle D^{2} \varphi(\hat{x})(x-\hat{x}), x-\hat{x}\right\rangle+o\left(|x-\hat{x}|^{2}\right)
$$


imply that $\left(q+D \varphi(\hat{x}), Y+D^{2} \varphi(\hat{x})\right) \in J_{\mathscr{\theta}}^{2,+} u(\hat{x})$ and so $J_{\mathscr{\theta}}^{2,+}(u-\varphi)(\hat{x}) \subset$ $\left\{\left(p-D \varphi(\hat{x}), X-D^{2} \varphi(x)\right):(p, X) \in J_{\mathscr{\theta}}^{2,+} u(x)\right\}$. The other inclusion follows from this as well, since $J_{\mathscr{Q}}^{2,+} u(\hat{x})=J_{\mathscr{Q}}^{2,+}((u-\varphi)+\varphi)(\hat{x})$. It is also clear that one always has

$$
J_{\mathscr{O}}^{2,+}(u+v)(x) \supset J_{\mathscr{\theta}}^{2,+} u(x)+J_{\mathscr{\theta}}^{2,+} v(x) .
$$

(iii) We consider $J_{\mathscr{\theta}}^{2,+} \varphi(\hat{x})$ when $\varphi \in C^{2}\left(\mathbb{R}^{N}\right)$ and will end up with a general statement corresponding to Example 2.1. In view of (ii), we may as well assume that $\varphi \equiv 0$, and we will write "Zero" for the zero function. We know that if $\hat{x} \in$ interior $(\mathscr{O})$, then $\left.J_{\mathscr{Q}}^{2,+} \operatorname{Zero}(\hat{x})=J^{2,+} \operatorname{Zero}(\hat{x})=\{(0, X)\}: X \geq 0\right\}$. In general, $(p, X) \in J_{\mathscr{\theta}}^{2,+} \operatorname{Zero}(\hat{x})$ if

$$
0 \leq\langle p, x-\hat{x}\rangle+\frac{1}{2}\langle X(x-\hat{x}), x-\hat{x}\rangle+o\left(|x-\hat{x}|^{2}\right) \quad \text { as } \mathscr{O} \ni x \rightarrow \hat{x} .
$$

Assuming that $x_{n} \in \mathscr{O}, 0<\left|x_{n}-\hat{x}\right| \rightarrow 0$, and $\left(x_{n}-\hat{x}\right) /\left|x_{n}-\hat{x}\right| \rightarrow q$, we may put $x=x_{n}$ in (2.9), divide the result by $\left|x_{n}-\hat{x}\right|$ and pass to the limit to conclude that

$$
0 \leq\langle p, q\rangle \text { for } q \in \mathrm{UT}_{\mathscr{O}}(\hat{x})
$$

where $\mathrm{UT}_{\mathscr{O}}(\hat{x})$ is the set of "generalized unit tangents" to $\mathscr{O}$ at $\hat{x}$; it is given by

$$
\mathrm{UT}_{\mathscr{O}}(\hat{x})=\left\{q: \exists x_{n} \in \mathscr{O} \backslash\{\hat{x}\}, \quad x_{n} \rightarrow \hat{x}, \quad \text { and } \frac{x_{n}-\hat{x}}{\left|x_{n}-\hat{x}\right|} \rightarrow q\right\} .
$$

If $\mathscr{O}$ is a smooth $N$-submanifold of $\mathbb{R}^{N}$ with boundary and $\hat{x} \in \partial \mathscr{O}$, then the generalized tangent cone

$$
T_{\mathscr{O}}(\hat{x})=\operatorname{convex} \operatorname{hull}\left(\mathrm{UT}_{\mathscr{O}}(\hat{x})\right)
$$

is a halfspace and $\mathscr{O}$ has an exterior normal $\vec{n}$ at $\hat{x}$. In this event, (2.10) says that $p=-\lambda \vec{n}$ for some $\lambda \geq 0$. Moreover, if $p=0,(2.9)$ then implies that $0 \leq\langle X q, q\rangle$ for $q \in \mathrm{UT}_{\mathscr{O}}(\hat{x})$, and we conclude that

$$
(0, X) \in J_{\mathscr{\theta}}^{2,+} \operatorname{Zero}(\hat{x}) \text { if and only if } 0 \leq X
$$

provided $T_{\mathscr{O}}(\hat{x})$ is a halfspace.

Life is more complex if $p=-\lambda \vec{n}$ and $\lambda>0$. In this case, (2.9) reads

$$
\lambda\langle\vec{n}, x-\hat{x}\rangle-\frac{1}{2}\langle X(x-\hat{x}), x-\hat{x}\rangle \leq o\left(|x-\hat{x}|^{2}\right) \quad \text { as } \mathscr{O} \ni x \rightarrow \hat{x} .
$$

We study (2.13) when $\hat{x}=0$ and we can represent $\mathscr{O}$ near 0 in the form

$$
\left\{\left(\tilde{x}, x_{N}\right): x_{N} \leq g(\tilde{x})\right\}
$$

where $\left.\tilde{x}=\left(x_{1}, \ldots, x_{N-1}\right), g(\tilde{x})=\frac{1}{2}\langle Z \tilde{x}, \tilde{x}\rangle+o\left(|\tilde{x}|^{2}\right)\right\}$, and $Z \in \mathscr{S}(N-1)$. That is, we assume that the boundary of $\mathscr{O}$ is twice differentiable at 0 and rotate so that the normal is $\vec{n}=e_{N}=(0, \ldots, 0,1)$. With these normalizations, we put $(\tilde{x}, g(\tilde{x}))$ into $(2.13)$ to find $\lambda\langle Z \tilde{x}, \tilde{x}\rangle-\langle X(\tilde{x}, 0),(\tilde{x}, 0)\rangle \leq o\left(|\tilde{x}|^{2}\right)$ or $\lambda \widetilde{Z} \leq P_{N-1} X P_{N-1}$ where $\tilde{Z}\left(\tilde{x}, x_{n}\right)=(Z \tilde{x}, 0)$ and $P_{N-1}$ is the projection on the first $N-1$ coordinates. It is not hard to see that this is also sufficient.

When unraveled, the above considerations lead to the following conclusion. Let $\mathscr{O}$ be an $N$-submanifold of $\mathbb{R}^{N}$ with boundary, $\hat{x} \in \partial \mathscr{O}, \partial \mathscr{O}$ be twice differentiable at $\hat{x}, \vec{n}$ be the outward normal, $T_{\partial \mathscr{O}}(\hat{x})$ be the tangent plane 
to $\partial \mathscr{O}$ at $\hat{x}$, and $P \in \mathscr{S}(N)$ be orthogonal projection on $T_{\partial \mathscr{O}}(\hat{x})$ (which we regard as a subspace of $\mathbb{R}^{N}$ ). Finally, let $S$ be the symmetric operator in $T_{\partial \mathscr{O}}(\hat{x})$ corresponding to the second fundamental form of $\partial \mathscr{O}$ at $\hat{x}$ (oriented with the exterior normal to $\mathscr{O}$ ) extended to $\mathbb{R}^{N}$ by $S \vec{n}=0$. Then

$$
\begin{cases}(p, X) \in J_{\mathscr{O}}^{2,+} \varphi(\hat{x}) & \text { if and only if either } p=D \varphi(\hat{x}) \text { and } D^{2} \varphi(\hat{x}) \leq X \text {, or } \\ p=D \varphi(\hat{x})-\lambda \vec{n}, & \lambda>0 \text { and } P D^{2} \varphi(\hat{x}) P \leq P X P-\lambda S .\end{cases}
$$

Noting that $P \vec{n} \otimes \vec{n} P=0$, we see that if $(p, X) \in J_{\mathscr{\theta}}^{2,+} u(\hat{x}), S \leq 0$, and $\lambda>0$, then $(p-\lambda \vec{n}, X+\mu \vec{n} \otimes \vec{n}) \in J_{\Theta}^{2,+} u(\hat{x})$ for $\mu \in \mathbb{R}$. For later use, we denote by $X_{\lambda}$ the particular choice of $X$ such that $(D \varphi(\hat{x})-\lambda \vec{n}, X) \in J_{\mathscr{Q}}^{2,+} \varphi(\hat{x})$ given by

$$
X_{\lambda}(\xi+\beta \vec{n})=D^{2} \varphi(\hat{x})(\xi+\beta \vec{n})+\lambda S \xi \text { for } \xi \in T_{\partial \mathscr{Q}}(\hat{x}), \beta \in \mathbb{R} .
$$

Before leaving this topic, we note that the set of exterior "normals"

$$
N(\hat{x})=\left(\vec{n} \in \mathbb{R}^{N}:\langle\vec{n}, q\rangle \leq 0 \text { for } q \in T_{\mathscr{O}}(\hat{x})\right)
$$

can also be described by

$$
N(\hat{x})=\left(\vec{n} \in \mathbb{R}^{N}:\langle\vec{n}, x-\hat{x}\rangle \leq o(|x-\hat{x}|) \text { as } \mathscr{O} \ni x \rightarrow \hat{x}\right)
$$

no matter how regular $\partial \mathscr{O}$ is at $\hat{x}$ and that we showed above that

$$
J_{\mathscr{O}}^{2,+} \varphi(\hat{x}) \subset(D \varphi(\hat{x})-N(\hat{x})) \times \mathscr{S}(N) .
$$

Notes on $\$ 2$. Viscosity solutions were introduced by M. G. Crandall and P. L. Lions [51] (following an announcement in [50]); analogies with S. N. Kruzkov's theory of scalar conservation laws [107] provided guidance for the notion and its presentation. The presentation of [51] emphasized the first-order case, owing to the fact that uniqueness results were only obtainable for this case at the time. It was also pointed out in [51] that there were several equivalent ways to formulate the notion of viscosity solutions; one of these was intimately connected with earlier work by L. C. Evans [64, 65] concerning "weak passages to the limit" in equations satisfying the maximum principle. In these developments, directions were indicated by aspects of nonlinear functional analysis and nonlinear semigroup theory.

It became apparent that working with these alternative formulations was superior to the approach taken in [50], and perhaps M. G. Crandall, L. C. Evans, and P. L. Lions [47] is the first readable account of the early theory. (We note that the definition most emphasized in [47] is not the one put foremost here.)

The necessity of defining the semijets on closed sets became apparent with the investigation of boundary value problems. We return to this issue in $\S 7$, but a couple of significant papers here are P. L. Lions [121] and H. M. Soner [149] (see also M. G. Crandall and R. Newcomb [58]). The closure of the semijets was introduced in H. Ishii and P. L. Lions [96] and was fully exploited in M. G. Crandall [50] and M. G. Crandall and H. Ishii [48]. Analogous operations have long been employed by "nonsmooth analysts."

The fact that the theory required only appropriate semicontinuity was realized earlier on, but the first striking applications of the fact (to existence, stability, optimal control, ...) were given in H. Ishii [86, 90], G. Barles and B. Perthame [25, 26]. 
Let us finally mention that some structural properties of viscosity solutions were and will be ommitted from our presentation and, in addition to the above references, some can be found in P. L. Lions [124], R. Jensen and P. E. Souganidis [105], and H. Frankowska [78].

\section{THE MAXIMUM PRINCIPLE FOR SEMICONTINUOUS FUNCTIONS AND COMPARISON FOR THE DIRICHLET PROBLEM}

Let $\Omega$ be a bounded open subset of $\mathbb{R}^{N}, \bar{\Omega}$ be its closure, and $\partial \Omega$ be its boundary. Suppose $u \in \operatorname{USC}(\bar{\Omega})$ is a solution of $F \leq 0$ in $\Omega, v \in \operatorname{LSC}(\bar{\Omega})$ is a solution of $F \geq 0$, and $u \leq v$ on $\partial \Omega$. We seek to show that $u \leq v$ on $\bar{\Omega}$.

In the event $u$ and $v$ are classical sub and supersolutions, we could employ the classical "maximum principle." Let us recall some elementary facts in this regard. Let $w$ be defined in a neighborhood of $\hat{x} \in \mathbb{R}^{N}$. If there exists $(p, X) \in$ $\mathbb{R}^{N} \times \mathscr{S}(N)$ such that

$$
w(x)=w(\hat{x})+\langle p, x-\hat{x}\rangle+\frac{1}{2}\langle X(x-\hat{x}), x-\hat{x}\rangle+o\left(|x-\hat{x}|^{2}\right) \text { as } x \rightarrow \hat{x}
$$

we say that $w$ is twice differentiable at $\hat{x}$ and $D w(\hat{x})=p, D^{2} w(\hat{x})=X$ (it being obvious that $p$ and $X$ are unique if they exist). It is clear that $w$ is twice differentiable at $\hat{x}$ if and only if $J^{2} w(\hat{x}) \equiv J^{2,+} w(\hat{x}) \cap J^{2,-} w(\hat{x})$ is nonempty (in which case $\left.J^{2} w(\hat{x})=\left\{\left(D w(\hat{x}), D^{2} w(\hat{x})\right)\right\}\right)$. Classical implementations of the maximum principle are based on the fact, already used above, that if $w$ is twice differentiable at a local maximum $\hat{x}$, then $D w(\hat{x})=0$ and $D^{2} w(\hat{x}) \leq 0$. Thus, if $u$ and $v$ are twice differentiable everywhere and $w=u-v$ has a local maximum $\hat{x} \in \Omega$, we would have $D u(\hat{x})=D v(\hat{x})$ and $D^{2} u(\hat{x}) \leq D^{2} v(\hat{x})$ and then, in view of $(0.1)$,

$$
\begin{aligned}
F\left(\hat{x}, u(\hat{x}), D u(\hat{x}), D^{2} u(\hat{x})\right) & \leq 0 \leq F\left(\hat{x}, v(\hat{x}), D v(\hat{x}), D^{2} v(\hat{x})\right) \\
& \leq F\left(\hat{x}, v(\hat{x}), D u(\hat{x}), D^{2} u(\hat{x})\right) .
\end{aligned}
$$

In the event that $F(x, r, p, X)$ is strictly nondecreasing in $r$ (a simple but illustrative case), it follows that $u-v$ is nonpositive at an interior maximum and so $u \leq v$ in $\Omega$ since $u \leq v$ holds on $\partial \Omega$.

We seek to extend this argument to the case $u \in \operatorname{USC}(\bar{\Omega}), v \in \operatorname{LSC}(\bar{\Omega})$. We are unable to simply plug $\left(D u(\hat{x}), D^{2} u(\hat{x})\right)$ and $\left(D v(\hat{x}), D^{2} v(\hat{x})\right)$ into $F$ since these expressions must be replaced by the set-valued functions $J^{2,+} u$ and $J^{2,-} v$ (and their values may well be empty at many points, including maximum points of $u(x)-v(x))$. To use $J^{2,+} u$ and $J^{2,-} v$, we employ a device that doubles the number of variables and then penalizes this doubling. More precisely, we maximize the function $u(x)-v(y)-(\alpha / 2)|x-y|^{2}$ over $\bar{\Omega} \times \bar{\Omega}$; here $\alpha>0$ is a parameter. As $\alpha \rightarrow \infty$, we closely approximate maximizing $u(x)-v(x)$ over $\bar{\Omega}$. More precisely, we have

Lemma 3.1. Let $\mathscr{O}$ be a subset of $\mathbb{R}^{N}, u \in \operatorname{USC}(\mathscr{O}), v \in \operatorname{LSC}(\mathscr{O})$ and

$$
M_{\alpha}=\sup _{\mathscr{\theta} \times \mathscr{\theta}}\left(u(x)-v(y)-\frac{\alpha}{2}|x-y|^{2}\right)
$$

for $\alpha>0$. Let $M_{\alpha}<\infty$ for large $\alpha$ and $\left(x_{\alpha}, y_{\alpha}\right)$ be such that

$$
\lim _{\alpha \rightarrow \infty}\left(M_{\alpha}-\left(u\left(x_{\alpha}\right)-v\left(y_{\alpha}\right)-\frac{\alpha}{2}\left|x_{\alpha}-y_{\alpha}\right|^{2}\right)\right)=0 .
$$


Then the following holds:

$$
\left\{\begin{array}{l}
\text { (i) } \lim _{\alpha \rightarrow \infty} \alpha\left|x_{\alpha}-y_{\alpha}\right|^{2}=0 \text { and } \\
\text { (ii) } \lim _{\alpha \rightarrow \infty} M_{\alpha}=u(\hat{x})-v(\hat{x})=\sup _{\mathscr{O}}(u(x)-v(x)) \\
\text { whenever } \hat{x} \in \mathscr{O} \text { is a limit point of } x_{\alpha} \text { as } \alpha \rightarrow \infty .
\end{array}\right.
$$

Deferring the elementary proof of the lemma and returning to our sub and supersolutions $u \in \operatorname{USC}(\bar{\Omega}), v \in \operatorname{LSC}(\bar{\Omega})$ satisfying $u \leq v$ on $\partial \Omega$, we note that $M_{\alpha}=\sup _{\bar{\Omega} \times \bar{\Omega}}\left(u(x)-v(y)-(\alpha / 2)|x-y|^{2}\right)$ is finite since $u(x)-v(y)$ is upper semicontinuous and $\bar{\Omega}$ is compact. Since we seek to prove $u \leq v$, we assume to the contrary that $u(z)>v(z)$ for some $z \in \Omega$; it follows that

$$
M_{\alpha} \geq u(z)-v(z)=\delta>0 \text { for } \alpha>0 .
$$

Choosing $\left(x_{\alpha}, y_{\alpha}\right)$ so that $M_{\alpha}=u\left(x_{\alpha}\right)-v\left(y_{\alpha}\right)-(\alpha / 2)\left|x_{\alpha}-y_{\alpha}\right|^{2}$ (the maximum is achieved in view of upper semicontinuity and compactness), it follows from (3.4)(i), (ii) and $u \leq v$ on $\partial \Omega$ that $\left(x_{\alpha}, y_{\alpha}\right) \in \Omega \times \Omega$ for $\alpha$ large. The next step is to use the equations to estimate $M_{\alpha}$ and contradict (3.5) for large $\alpha$. This requires producing suitable values of $J^{2,+} u$ and $J^{2,-} v$, and we turn to this question.

To know what to look for, let us proceed more generally and assume that $u$, $v$ are defined in neighborhoods of $\hat{x}, \hat{y} \in \mathbb{R}^{N}$ and twice differentiable at $\hat{x}, \hat{y}$ respectively. Assume, moreover, that $\varphi$ is $C^{2}$ near $(\hat{x}, \hat{y})$ in $\mathbb{R}^{N} \times \mathbb{R}^{N}$ and $(\hat{x}, \hat{y})$ is a local maximum of $u(x)-v(y)-\varphi(x, y)$. Applying the classical maximum principle to this situation (in the $2 \mathrm{~N}$ variables $(x, y)$ ), we learn that $D u(\hat{x})=D_{x} \varphi(\hat{x}, \hat{y}), D v(\hat{y})=-D_{y} \varphi(\hat{x}, \hat{y})$, and

$$
\left(\begin{array}{cc}
X & 0 \\
0 & -Y
\end{array}\right) \leq D^{2} \varphi(\hat{x}, \hat{y})
$$

where $X=D^{2} u(\hat{x}), \quad Y=D^{2} v(\hat{y})$. Note that with the choice $\varphi(x, y)=$ $(\alpha / 2)|x-y|^{2}$ the above reads

$$
\left(\begin{array}{cc}
X & 0 \\
0 & -Y
\end{array}\right) \leq \alpha\left(\begin{array}{cc}
I & -I \\
-I & I
\end{array}\right)
$$

where $I$ will stand for the identity matrix in any dimension and, since the right-hand side annihilates vectors of the form $\left(\begin{array}{l}\xi \\ \xi\end{array}\right)$ (also written ${ }^{t}(\xi, \xi)$ where ${ }^{t} Z$ denotes the transpose of a matrix $Z$ ), (3.7) implies $X \leq Y$, making further contact with the maximum principle.

It is a remarkable fact that perturbations of the above results may be obtained in the class of semicontinuous functions. The main result we use in this direction is the following theorem, which is formulated in a useful but distracting generality. (See (2.6) and (2.7) regarding notation below.)

Theorem 3.2. Let $\mathscr{O}_{i}$ be a locally compact subset of $R^{N_{i}}$ for $i=1, \ldots, k$,

$$
\mathscr{O}=\mathscr{O}_{1} \times \cdots \times \mathscr{O}_{k},
$$

$u_{i} \in \operatorname{USC}\left(\mathscr{O}_{i}\right)$, and $\varphi$ be twice continuously differentiable in a neighborhood of O. Set

$$
w(x)=u_{1}\left(x_{1}\right)+\cdots+u_{k}\left(x_{k}\right) \text { for } x=\left(x_{1}, \cdots, x_{k}\right) \in \mathscr{O}
$$


and suppose $\hat{x}=\left(\hat{x}_{1}, \ldots, \hat{x}_{k}\right) \in \mathscr{O}$ is a local maximum of $w-\varphi$ relative to O. Then for each $\varepsilon>0$ there exists $X_{i} \in \mathscr{S}\left(N_{i}\right)$ such that

$$
\left(D_{x_{i}} \varphi(\hat{x}), X_{i}\right) \in \bar{J}_{\hat{O}_{i}}^{2+} u_{i}\left(\hat{x}_{i}\right) \text { for } i=1, \ldots, k,
$$

and the block diagonal matrix with entries $X_{i}$ satisfies

$$
-\left(\frac{1}{\varepsilon}+\|A\|\right) I \leq\left(\begin{array}{ccc}
X_{1} & \ldots & 0 \\
\vdots & \ddots & \vdots \\
0 & \ldots & X_{k}
\end{array}\right) \leq A+\varepsilon A^{2}
$$

where $A=D^{2} \varphi(\hat{x}) \in \mathscr{S}(N), N=N_{1}+\cdots+N_{k}$.

The norm of the symmetric matrix $A$ used in (3.8) is

$$
\|A\|=\sup \{|\lambda|: \lambda \text { is an eigenvalue of } A\}=\sup \{|\langle A \xi, \xi\rangle|:|\xi| \leq 1\} .
$$

We caution the reader that this result is an efficient summarization of the analytical heart of the theory suitable for the presentation we have chosen and its proof, which is outlined in the appendix, is deeper and more difficult than its applications that we give in the main text. See also the notes to this section.

In order to apply Theorem 3.2 in the above situation, we put $k=2, \mathscr{O}_{1}=$ $\mathscr{O}_{2}=\Omega, u_{1}=u, u_{2}=-v, \varphi(x, y)=(\alpha / 2)|x-y|^{2}$, and recall that $\bar{J}_{\Omega}^{2,-} v=$ $-\bar{J}_{\Omega}^{2,+}(-v)$. In this case

$$
\begin{gathered}
D_{x} \varphi(\hat{x}, \hat{y})=-D_{y} \varphi(\hat{x}, \hat{y})=\alpha(\hat{x}-\hat{y}), \quad A=\alpha\left(\begin{array}{cc}
I & -I \\
-I & I
\end{array}\right), \\
A^{2}=2 \alpha A, \quad\|A\|=2 \alpha,
\end{gathered}
$$

and we conclude that for every $\varepsilon>0$ there exists $X, Y \in \mathscr{S}(N)$ such that

$$
(\alpha(\hat{x}-\hat{y}), X) \in \bar{J}_{\Omega}^{2,+} u(\hat{x}), \quad(\alpha(\hat{x}-\hat{y}), Y) \in \bar{J}_{\Omega}^{2,-} v(\hat{y})
$$

and

$$
-\left(\frac{1}{\varepsilon}+2 \alpha\right)\left(\begin{array}{cc}
I & 0 \\
0 & I
\end{array}\right) \leq\left(\begin{array}{cc}
X & 0 \\
0 & -Y
\end{array}\right) \leq \alpha(1+2 \varepsilon \alpha)\left(\begin{array}{cc}
I & -I \\
-I & I
\end{array}\right) .
$$

Choosing $\varepsilon=1 / \alpha$ yields the elegant relations

$$
-3 \alpha\left(\begin{array}{cc}
I & 0 \\
0 & I
\end{array}\right) \leq\left(\begin{array}{cc}
X & 0 \\
0 & -Y
\end{array}\right) \leq 3 \alpha\left(\begin{array}{cc}
I & -I \\
-I & I
\end{array}\right)
$$

in all, we conclude that at a maximum $(\hat{x}, \hat{y})$ of $u(x)-v(y)-(\alpha / 2)|x-y|^{2}$ there exists $X,-Y \in \mathscr{S}(N)$ such that (3.9) and (3.10) hold. Observe that, in comparison with (3.7), we have worsened the upper bound by a factor of 3 (but can still conclude from (3.10) that $X \leq Y$ ) and obtained a lower bound. We will not actually use the lower bound in what follows, but its presence corresponds to an essential compactness issue in the proof of the theorem.

Let us finish this chapter of the story. Writing $(\hat{x}, \hat{y})$ in place of $\left(x_{\alpha}, y_{\alpha}\right)$ for simplicity, we only need to assume that

$$
F(\hat{x}, u(\hat{x}), \alpha(\hat{x}-\hat{y}), X) \leq 0 \leq F(\hat{y}, v(\hat{y}), \alpha(\hat{x}-\hat{y}), Y)
$$

is inconsistent with the other information we have in hand, which includes

$$
\left\{\begin{array}{l}
0<\delta \leq M_{\alpha}=u(\hat{x})-v(\hat{y})-(\alpha / 2)|\hat{x}-\hat{y}|^{2} \leq u(\hat{x})-v(\hat{y}) \text { and } \\
\alpha|\hat{x}-\hat{y}|^{2} \rightarrow 0 \text { as } \alpha \rightarrow \infty
\end{array}\right.
$$


(the second assertion follows from (3.4)(i)) and (3.10). For example, assume that there exists $\gamma>0$ such that

$\gamma(r-s) \leq F(x, r, p, X)-F(x, s, p, X)$ for $r \geq s,(x, p, X) \in \bar{\Omega} \times \mathbb{R}^{N} \times \mathscr{S}(N)$

and there is a function $\omega:[0, \infty] \rightarrow[0, \infty]$ that satisfies $\omega(0+)=0$ such that (3.14)

$$
\left\{\begin{array}{l}
F(y, r, \alpha(x-y), Y)-F(x, r, \alpha(x-y), X) \leq \omega\left(\alpha|x-y|^{2}+|x-y|\right) \\
\text { whenever } x, y \in \Omega, r \in \mathbb{R}, X, Y \in \mathscr{S}(N), \text { and (3.10) holds. }
\end{array}\right.
$$

Proceding, we deduce from (3.12), (3.13), (3.11), and (3.14) that $(3.15)$

$$
\begin{aligned}
\gamma \delta \leq & \gamma(u(\hat{x})-v(\hat{y})) \leq F(\hat{x}, u(\hat{x}), \alpha(\hat{x}-\hat{y}), X)-F(\hat{x}, v(\hat{y}), \alpha(\hat{x}-\hat{y}), X) \\
= & (F(\hat{x}, u(\hat{x}), \alpha(\hat{x}-\hat{y}), X)-F(\hat{y}, v(\hat{y}), \alpha(\hat{x}-\hat{y}), Y)) \\
& +(F(\hat{y}, v(\hat{y}), \alpha(\hat{x}-\hat{y}), Y)-F(\hat{x}, v(\hat{y}), \alpha(\hat{x}-\hat{y}), X)) \\
& \leq \omega\left(\alpha|\hat{x}-\hat{y}|^{2}+|\hat{x}-\hat{y}|\right) ;
\end{aligned}
$$

here we used (3.11) to estimate the first term on the right by 0 and (3.14) on the second term. Since $\omega\left(\alpha|\hat{x}-\hat{y}|^{2}+|\hat{x}-\hat{y}|\right) \rightarrow 0$ as $\alpha \rightarrow \infty$ by (3.12), we have a contradiction. We have proved

Theorem 3.3. Let $\Omega$ be a bounded open subset of $\mathbb{R}^{N}, F \in C\left(\Omega \times \mathbb{R} \times \mathbb{R}^{N} \times\right.$ $\mathscr{S}(N)$ ) be proper and satisfy (3.13), (3.14). Let $u \in \operatorname{USC}(\bar{\Omega})$ (respectively, $v \in \operatorname{LSC}(\bar{\Omega})$ ) be a subsolution (respectively, supersolution) of $F=0$ in $\Omega$ and $u \leq v$ on $\partial \Omega$. Then $u \leq v$ in $\bar{\Omega}$.

Remark 3.4. We motivated the structure condition (3.14) by the natural way it arose in the course of analysis and (3.14) was used in the proof and not degenerate ellipticity. However, (3.14) implies degenerate ellipticity. To see this, suppose that (3.14) holds, $X, Y \in \mathscr{S}(N)$ and $X \leq Y$. Observe that then for $\xi, \eta \in \mathbb{R}^{N}$, and $\varepsilon>0$

$$
\begin{aligned}
\langle X \xi, \xi\rangle-\langle Y \eta, \eta\rangle & \leq\langle Y \xi, \xi\rangle-\langle Y \eta, \eta\rangle=2\langle Y \eta, \xi-\eta\rangle+\langle Y(\xi-\eta), \xi-\eta\rangle \\
& \leq \varepsilon|\eta|^{2}+\left(1+\frac{\|Y\|}{\varepsilon}\right)\|Y\||\xi-\eta|^{2} .
\end{aligned}
$$

The last inequality may be written

$$
\left(\begin{array}{cc}
X & 0 \\
0 & -(Y+\varepsilon I)
\end{array}\right) \leq\left(1+\frac{\|Y\|}{\varepsilon}\right)\|Y\|\left(\begin{array}{cc}
I & -I \\
-I & I
\end{array}\right) .
$$

Thus if $\alpha>(1 / 3) \max \{\|X\|,\|Y\|,(1+\|Y\| / \varepsilon)\|Y\|\}$ and $\varepsilon$ is sufficiently small, the pair $X, Y+\varepsilon I$ satisfy (3.10). Next we fix $x, r$, and $p$, and put $y=x-p / \alpha$ in (3.14) so that by $(3.14)$

$$
F\left(x-\frac{p}{\alpha}, r, p, Y+\varepsilon I\right)-F(x, r, p, X) \leq \omega\left(\frac{1}{\alpha}\left(|p|^{2}+|p|\right)\right)
$$

and then we let $\alpha \rightarrow \infty$ and $\varepsilon \downarrow 0$ and conclude that $F$ is degenerate elliptic. Remark 3.5. The proof of Theorem 3.3 adapts to provide modulus of continuity estimates on solutions of $F=0$. 
Example 3.6. We turn to some examples in which (3.14) is satisfied. First notice that (3.14) evidently holds (with $\omega$ the modulus of continuity for $f$ ) for $F(x, r, p, X)=G(r, p, X)-f(x)$ if $G$ is degenerate elliptic and $f$ is continuous; this is because (3.10) implies $X \leq Y$.

Secondly, the linear first order expression $\langle b(x), p\rangle$ satisfies (3.14) if

$$
\alpha\langle b(y)-b(x), x-y\rangle \leq \omega\left(\alpha|x-y|^{2}+|x-y|\right)
$$

for some $\omega$ and this holds with $\omega(r)=c r$ if there is a constant $c>0$ such that $\langle b(x)-b(y), x-y\rangle \geq-c|x-y|^{2}$, i.e., the vector field $b+c I$ is "monotone." In fact, it is not hard to see that this is a necessary condition as well.

Next, the linear expression

$$
G(x, X)=-\operatorname{trace}\left({ }^{t} \Sigma(x) \Sigma(x) X\right),
$$

where $\Sigma$ maps $\bar{\Omega}$ into the $N \times N$ real matrices, is degenerate elliptic (it is a special case of Example 1.2) with $\left.A(x)={ }^{t} \Sigma(x) \Sigma(x)\right)$. We seek to estimate $G(y, Y)-G(x, X)$ when (3.10) holds. Multiplying the rightmost inequality in (3.10) by the nonnegative symmetric matrix

$$
\left(\begin{array}{cc}
t^{t}(x) \Sigma(x) & \left.{ }^{t} \Sigma(y) \Sigma(x)\right) \\
{ }^{t} \Sigma(x) \Sigma(y) & { }^{t} \Sigma(y) \Sigma(y)
\end{array}\right)
$$

and taking traces preserves the inequality and yields

$$
\begin{aligned}
G(y, Y)-G(x, X) & =\operatorname{trace}\left({ }^{t} \Sigma(x) \Sigma(x) X-{ }^{t} \Sigma(y) \Sigma(y) Y\right) \\
& \leq 3 \alpha \operatorname{trace}\left(\left({ }^{t} \Sigma(x)-{ }^{t} \Sigma(y)\right)(\Sigma(x)-\Sigma(y)),\right.
\end{aligned}
$$

so if $\Sigma$ is Lipschitz continuous with constant $L$ then

$$
G(y, Y)-G(x, X) \leq 3 L^{2} \alpha|x-y|^{2}
$$

and we may choose $\omega(r)=3 L^{2} r$. We note that if $\Gamma(x): \bar{\Omega} \rightarrow \mathscr{S}(N)$ is Lipschitz continuous and $\Gamma(x)>0$ in $\bar{\Omega}$, then

$$
-\operatorname{trace}(\Gamma(x) X)=-\operatorname{trace}\left(\Gamma(x)^{1 / 2} \Gamma(x)^{1 / 2} X\right)
$$

is an example of the sort just discussed since $\Gamma(x)^{1 / 2}$ is also Lipschitz continuous. Finally, it is known that it $\Gamma(x) \geq 0$ and $\Gamma \in W^{2, \infty}$, then $\Gamma^{1 / 2}$ is Lipschitz continuous.

Now we note that the processes of forming sums or sups or sup infs, etc., above produce examples obeying (3.14) if the ingredients obey (3.14) with a common $\omega$.

Finally, we may produce examples satisfying (3.13) and (3.14) by putting

$$
F(x, r, p, X)=\gamma r+G(x, r, p, X)
$$

whenever $G$ is degenerate elliptic and satisfies (3.14).

We deferred discussion of the elementary Lemma 3.1 since it is better presented in a generality not required above. Indeed, Lemma 3.1 may be obtained from the following proposition via the correspondences $2 N \rightarrow M, \mathscr{O} \times \mathscr{O} \rightarrow$ $\mathscr{O},(x, y) \rightarrow x, u(x)-v(y) \rightarrow \Phi(x),(1 / 2)|x-y|^{2} \rightarrow \Psi(x)$. 
Proposition 3.7. Let $\mathscr{O}$ be a subset of $\mathbb{R}^{M}, \Phi \in \operatorname{USC}(\mathscr{O}), \Psi \in \operatorname{LSC}(\mathscr{O}), \Psi \geq 0$, and

$$
M_{\alpha}=\sup _{\theta}(\Phi(x)-\alpha \Psi(x))
$$

for $\alpha>0$. Let $-\infty<\lim _{\alpha \rightarrow \infty} M_{\alpha}<\infty$ and $x_{\alpha} \in \mathscr{O}$ be chosen so that

$$
\lim _{\alpha \rightarrow \infty}\left(M_{\alpha}-\left(\Phi\left(x_{\alpha}\right)-\alpha \Psi\left(x_{\alpha}\right)\right)=0 .\right.
$$

Then the following hold:

$$
\left\{\begin{array}{l}
\text { (i) } \lim _{\alpha \rightarrow \infty} \alpha \Psi\left(x_{\alpha}\right)=0 \\
\text { (ii) } \Psi(\hat{x})=0 \text { and } \lim _{\alpha \rightarrow \infty} M_{\alpha}=\Phi(\hat{x})=\sup _{\{\Psi(x)=0\}} \Phi(x) \\
\text { whenever } \hat{x} \in \mathscr{O} \text { is a limit point of } x_{\alpha} \text { as } \alpha \rightarrow \infty .
\end{array}\right.
$$

Proof. Put

$$
\delta_{\alpha}=M_{\alpha}-\left(\Phi\left(x_{\alpha}\right)-\alpha \Psi\left(x_{\alpha}\right)\right)
$$

so that $\delta_{\alpha} \rightarrow 0$ as $\alpha \rightarrow \infty$. Since $\Psi \geq 0, M_{\alpha}$ decreases as $\alpha$ increases and $\lim _{\alpha \rightarrow \infty} M_{\alpha}$ exists; it is finite by assumption. Moreover,

$$
M_{\alpha / 2} \geq \Phi\left(x_{\alpha}\right)-\frac{\alpha}{2} \Psi\left(x_{\alpha}\right) \geq \Phi\left(x_{\alpha}\right)-\alpha \Psi\left(x_{\alpha}\right)+\frac{\alpha}{2} \Psi\left(x_{\alpha}\right) \geq M_{\alpha}-\delta_{\alpha}+\frac{\alpha}{2} \Psi\left(x_{\alpha}\right),
$$

so $2\left(M_{\alpha / 2}-M_{\alpha}+\delta_{\alpha}\right) \geq \alpha \Psi\left(x_{\alpha}\right)$, which shows that $\alpha \Psi\left(x_{\alpha}\right) \rightarrow 0$ as $\alpha \rightarrow \infty$. Suppose now that $\alpha_{n} \rightarrow \infty$ and $x_{\alpha_{n}} \rightarrow \hat{x} \in \mathscr{O}$. Then $\Psi\left(x_{\alpha_{n}}\right) \rightarrow 0$ and by the lower semicontinuity $\Psi(\hat{x})=0$. Since

$$
\Phi\left(x_{\alpha_{n}}\right)-\alpha_{n} \Psi\left(x_{\alpha_{n}}\right) \geq M_{\alpha_{n}}-\delta_{\alpha_{n}} \geq \sup _{\{\Psi=0\}} \Phi(x)-\delta_{\alpha_{n}}
$$

and $\Phi$ is upper semicontinuous, (3.20) holds.

Remark 3.8. We record, for later use, some observations concerning maximum points $(\hat{x}, \hat{y})$ of $u(x)-v(y)-\varphi(x-y)$ over $\mathscr{O} \times \mathscr{O}$ for other choices of $\varphi$ besides $(\alpha / 2)|x|^{2}$ and the implications of Theorem 3.2. We are assuming that $u$ is upper semicontinuous, $v$ is lower semicontinuous, and $\varphi \in C^{2}$. In this application, the matrix $A$ of Theorem 3.2 has the form

$$
A=\left(\begin{array}{cc}
Z & -Z \\
-Z & Z
\end{array}\right), \text { where } Z=\left(D^{2} \varphi\right)(\hat{x}-\hat{y}),
$$

so

$$
A^{2}=\left(\begin{array}{cc}
2 Z^{2} & -2 Z^{2} \\
-2 Z^{2} & 2 Z^{2}
\end{array}\right)
$$

and $\|A\|=2\|Z\|$. that

Choosing $\varepsilon=\frac{1}{\|A\|}$ in (3.8), we conclude that there are $X, Y \in \mathscr{S}(N)$ such

$$
(D \varphi(\hat{x}-\hat{y}), X) \in J_{\mathscr{\theta}}^{2,+} u(\hat{x}), \quad(D \varphi(\hat{x}-\hat{y}), Y) \in J_{\Omega}^{2,-} v(\hat{y}),
$$

and

$$
-2\|A\|\left(\begin{array}{cc}
I & 0 \\
0 & I
\end{array}\right) \leq\left(\begin{array}{cc}
X & 0 \\
0 & -Y
\end{array}\right) \leq\left(\begin{array}{cc}
Z+\frac{1}{\|A\|} Z^{2} & -\left(Z+\frac{1}{\|A\|} Z^{2}\right) \\
-\left(Z+\frac{1}{\|A\|} Z^{2}\right) & Z+\frac{1}{\|A\|} Z^{2}
\end{array}\right)
$$


in particular,

$$
\|X\|,\|Y\| \leq 2\|A\| \text { and } X \leq Y .
$$

Notes on $\S 3$. This section is self-contained except for the proof of Theorem 3.2, which is due to $M$. G. Crandall and H. Ishii [48]. This result distills and sharpens the essence of a line of development that runs through several of the references listed below; the proof is explained in the appendix for the reader's convenience. We want to emphasize at the outset that our presentation of the proof of the comparison theorem, based as it is on Theorem 3.2, is but one among several possibilities. Moreover, other approaches may be useful in other situations. For example, one may use elements of the proof of Theorem 3.2 directly and look at corresponding manipulations on solutions-this approach seems to be the leading presentation for the infinite-dimensional extensions of the theory and allows for adaptations on the test functions and the notion itself and avoids the "semijets" or generalized derivatives. Another presentation selects another building block, namely, regularization by supconvolutions (which occur in the proof of Theorem 3.2) and its effect on viscosity solutions; this procedure is especially helpful in some particular problems (integrodifferential equations, regularity issues) and stresses the regularization procedure. This procedure has some analogues with the use of mollification in the study of linear partial differential equations. With apologies for confusing the reader, we are saying that a full grasp of all the elements of the proof of Theorem 3.2 and exposure to other presentations (e.g., R. Jensen [102] and H. Ishii and P. L. Lions [96]) may prove valuable.

The first uniqueness proofs for viscosity solutions were given for first-order equations in M. G. Crandall and P. L. Lions [51] and then M. G. Crandall, L. C. Evans, and P. L. Lions [47]. The second-order case remained open for quite a while during which the only evidence that a general theory could be developed was in results for Hamilton-Jacobi-Bellman equations obtained by P. L. Lions [119, 118]. The proof in these works involved ad hoc stochastic control verification arguments.

A breakthrough was achieved in the second-order theory by $\mathbf{R}$. Jensen [101] with the introduction of several key arguments; some of these were simplified in R. Jensen, P. L. Lions, and P. E. Souganidis [104], P. L. Lions and P. E. Souganidis [132], and R. Jensen [102]. In particular, the use of the "supconvolution" regularization (see the appendix), which is a standard tool in convex and nonsmooth analysis, was somewhat "remise au goût dujour" by J. M. Lasry and P. L. Lions [110].

Progress in understanding these proofs so as to be able to handle more examples was made by $H$. Ishii [89] who introduced matrix inequalities of the general form (3.7). This work contains an example (following [89, Theorem 3.3]) showing the optimality of condition (3.14) in the sense that we cannot replace the right-hand side of (3.14) by, for example, $\omega\left(\alpha|x-y|^{\theta}+|x-y|\right)$ with $\theta<2$ (but see §5.A). Estimating the left-hand side of (3.14) differently, for example in the form $\alpha g(|x-y|)+|x-y|$, it is possible to prove uniqueness in cases in which $g(r) / r^{2}$ is unbounded near $r=0$ by refining the arguments of [49]. A more complete understanding together with the sharpest structure conditions (up to the present) were achieved in [96], which also contains many examples. We mention that the structure condition (3.14) exhibited in this section pays no 
attention to whether the equation is of first order or possess stronger ellipticity properties. In the case of uniformly ellipitic $F$, much more can be done [96]. See also Jensen [102] as regards structure conditions.

A useful sharpening and improved organization of the analytical essence of the theory were contributed by [46]. The presentation here was based on Theorem 3.2; as remarked above, it follows from [48] and the generality and proofs presented there represent the current state of the art (see the appendix).

\section{Perron's method AND existence}

Let $\Omega$ be an arbitrary open subset of $\mathbb{R}^{N}$. By a solution (respectively, subsolution, etc.) of the Dirichlet problem

$$
\left\{\begin{aligned}
F\left(x, u, D u, D^{2} u\right) & =0 & & \text { in } \Omega, \\
u & =0 & & \text { on } \partial \Omega
\end{aligned}\right.
$$

we mean a function $u \in C(\bar{\Omega})$ (respectively, $u \in \operatorname{USC}(\bar{\Omega})$, etc.) that is a (viscosity) solution (respectively, subsolution, etc.) of $F=0$ in $\Omega$ and satisfies $u(x)=0$ (respectively, $u(x) \leq 0$, etc.) for $x \in \partial \Omega$. (We note that this formulation imposes the boundary condition in a strict sense that will be relaxed in §7.)

Recall that we always assume that $F$ is proper and, unless otherwise said, continuous . To discuss Perron's method, we will use the following notations: if $u: \mathscr{\theta} \rightarrow[-\infty, \infty]$ where $\mathscr{\theta} \subset \mathbb{R}^{N}$, then

$$
\left\{\begin{array}{l}
u^{*}(x)=\lim _{r \downarrow 0} \sup \{u(y): y \in \mathscr{O} \text { and }|y-x| \leq r\}, \\
u_{*}(x)=\lim _{r \downarrow 0} \inf \{u(y): y \in \mathscr{O} \text { and }|y-x| \leq r\} .
\end{array}\right.
$$

One calls $u^{*}$ the upper semicontinuous envelope of $u$; it is the smallest upper semicontinuous function (with values in $[-\infty, \infty]$ ) satisfying $u \leq u^{*}$. Similarly, $u_{*}$ is the lower semicontinuous envelope of $u$.

Theorem 4.1. (Perron's Method). Let comparison hold for (DP); i.e., if $w$ is a subsolution of (DP) and $v$ is a supersolution of (DP), then $w \leq v$. Suppose also that there is a subsolution $\underline{u}$ and a supersolution $\bar{u}$ of (DP) that satisfy the boundary condition $\underline{u}_{*}(x)=\bar{u}^{*}(x)=0$ for $x \in \partial \Omega$. Then

$$
W(x)=\sup \{w(x): \underline{u} \leq w \leq \bar{u} \text { and } w \text { is a subsolution of (DP) }\}
$$

is a solution of $(\mathrm{DP})$.

Theorem 3.3 provides conditions under which comparison holds for (DP). The proof consists of two steps. The first one is

Lemma 4.2. Let $\mathscr{O} \subset \mathbb{R}^{N}$ be locally compact, $F \in \operatorname{LSC}\left(\mathscr{O} \times \mathbb{R} \times \mathbb{R}^{N} \times \mathscr{S}(N)\right)$, and $\mathscr{F}$ be a family of solutions of $F \leq 0$ in $\mathscr{O}$. Let $w(x)=\sup \{u(x): u \in \mathscr{F}\}$ and assume that $w^{*}(x)<\infty$ for $x \in \mathcal{O}$. Then $w^{*}$ is a solution of $F \leq 0$ in ()

Allowing $F$ to be merely lower semicontinuous does not affect the proof and is used later. The information produced while proving this lemma is needed later as well, so we isolate the essential point in another result. 
Proposition 4.3. Let $\mathscr{O} \subset \mathbb{R}^{N}$ be locally compact, $v \in \operatorname{USC}(\mathscr{O}), z \in \mathscr{O}$, and $(p, X) \in J_{\mathscr{\theta}}^{2,+} v(z)$. Suppose also that $u_{n}$ is a sequence of upper semicontinuous functions on $\mathcal{O}$ such that

$$
\left\{\begin{array}{l}
\text { (i) there exists } x_{n} \in \mathscr{O} \text { such that }\left(x_{n}, u_{n}\left(x_{n}\right)\right) \rightarrow(z, v(z)), \\
\text { (ii) if } z_{n} \in \mathscr{O} \text { and } z_{n} \rightarrow x \in \mathscr{O} \text {, then } \lim \sup _{n \rightarrow \infty} u_{n}\left(z_{n}\right) \leq v(x) .
\end{array}\right.
$$

Then

$$
\left\{\begin{array}{l}
\text { there exists } \hat{x}_{n} \in \mathcal{O},\left(p_{n}, X_{n}\right) \in J_{\mathscr{O}}^{2,+} u_{n}\left(\hat{x}_{n}\right) \\
\text { such that }\left(\hat{x}_{n}, u_{n}\left(\hat{x}_{n}\right), p_{n}, X_{n}\right) \rightarrow(z, v(z), p, X) .
\end{array}\right.
$$

Proof. Without loss of generality we put $z=0$. By the assumptions, for every $\delta>0$ there is an $r>0$ such that $N_{r}=\{x \in \mathscr{O}:|x| \leq r\}$ is compact and

$$
v(x) \leq v(0)+\langle p, x\rangle+\frac{1}{2}\langle X x, x\rangle+\delta|x|^{2} \text { for } x \in N_{r} .
$$

By the assumption (4.3)(i), there exists $x_{n} \in \mathscr{O}$ such that $\left(x_{n}, u_{n}\left(x_{n}\right)\right) \rightarrow$ $(0, v(0))$. Let $\hat{x}_{n} \in N_{r}$ be a maximum point of the function $u_{n}(x)-(\langle p, x\rangle+$ $\left.\frac{1}{2}\langle X x, x\rangle+2 \delta|x|^{2}\right)$ over $N_{r}$ so that

$$
\begin{array}{r}
u_{n}(x) \leq u_{n}\left(\hat{x}_{n}\right)+\left\langle p, x-\hat{x}_{n}\right\rangle+\frac{1}{2}\left(\langle X x, x\rangle-\left\langle X \hat{x}_{n}, \hat{x}_{n}\right\rangle\right)+2 \delta\left(|x|^{2}-\left|\hat{x}_{n}\right|^{2}\right) \\
\text { for } x \in N_{r} .
\end{array}
$$

Suppose that (passing to a subsequence if necessary) $\hat{x}_{n} \rightarrow y$ as $n \rightarrow \infty$. Putting $x=x_{n}$ in (4.6) and taking the limit inferior as $n \rightarrow \infty$, we find

$$
v(0) \leq \liminf _{n \rightarrow \infty} u_{n}\left(\hat{x}_{n}\right)-\langle p, y\rangle-\frac{1}{2}\langle X y, y\rangle-2 \delta|y|^{2}
$$

on the other hand, by (4.3)(ii) $\lim \inf u_{n}\left(\hat{x}_{n}\right) \leq v(y)$ while (4.5) implies $v(y)-$ $\langle p, y\rangle-\frac{1}{2}\langle X y, y\rangle-2 \delta|y|^{2} \leq v(0)-\delta|y|^{2}$. We conclude that

$$
\begin{aligned}
v(0) & \leq \liminf _{n \rightarrow \infty} u_{n}\left(\hat{x}_{n}\right)-\langle p, y\rangle-\frac{1}{2}\langle X y, y\rangle-2 \delta|y|^{2} \\
& \leq v(y)-\langle p, y\rangle-\frac{1}{2}\langle X y, y\rangle-2 \delta|y|^{2} \leq v(0)-\delta|y|^{2} .
\end{aligned}
$$

From the extreme inequalities we learn $y=0$, so $\hat{x}_{n} \rightarrow 0$ (without passing to a subsequence), and then from the first inequality and (4.3)(ii) one sees that $v(0)=\lim _{n \rightarrow \infty} u_{n}\left(\hat{x}_{n}\right)$. Since we have $\left(p+4 \delta \hat{x}_{n}+X \hat{x}_{n}, X+4 \delta I\right) \in$ $J_{\mathscr{\theta}}^{2,+} u_{n}\left(\hat{x}_{n}\right)$ for large $n$, we are nearly done. To conclude, we merely note that the set of $(q, Y) \in \mathbb{R}^{N} \times \mathscr{S}(N)$ such that there exists $z_{n} \in \mathscr{O},\left(p_{n}, X_{n}\right) \in$ $J_{\mathscr{\theta}}^{2,+} u_{n}\left(z_{n}\right)$ such that $\left(z_{n}, u_{n}\left(z_{n}\right), p_{n}, X_{n}\right) \rightarrow(0, v(0), q, Y)$ is closed and contains $(p, X+4 \delta I)$ for $\delta>0$ by the above.

Proof of Lemma 4.2. With the notation of the lemma, suppose that $z \in \mathcal{O}$ and $(p, X) \in J_{\mathscr{O}}^{2,+} w^{*}(z)$. We seek to show that $F\left(z, w^{*}(z), p, X\right) \leq 0$. It is clear that we may choose a sequence $\left(x_{n}, u_{n}\right) \in \mathscr{O} \times \mathscr{F}$ such that $\left(x_{n}, u_{n}\left(x_{n}\right)\right) \rightarrow$ $\left(z, w^{*}(z)\right)$ and that (4.3) then holds with $v=w^{*}$. Hence, by the existence of data satisfying (4.4) and the fact that each $u_{n}$ is a subsolution, we may pass to the limit in the relation $F\left(\hat{x}_{n}, u_{n}\left(\hat{x}_{n}\right), p_{n}, X_{n}\right) \leq 0$ to find $F\left(z, w^{*}(z), p, X\right)$ $\leq 0$ as desired.

The second step in the proof of Theorem 4.1 is a simple construction that we now describe. Suppose that $\Omega$ is open, $u$ is a solution of $F \leq 0$, and $u_{*}$ is not a solution of $F \geq 0$; in particular, assume $0 \in \Omega$ and we have

$$
F\left(0, u_{*}(0), p, X\right)<0 \text { for some }(p, X) \in J_{\Omega}^{2,-} u_{*}(0)
$$


Then, by continuity, $u_{\delta, \gamma}(x)=u_{*}(0)+\delta+\langle p, x\rangle+\frac{1}{2}\langle X x, x\rangle-\gamma|x|^{2}$ is a classical solution of $F \leq 0$ in $B_{r}=\{x:|x|<r\}$ for all small $r, \delta, \gamma>0$. Since

$$
u(x) \geq u_{*}(x) \geq u_{*}(0)+\langle p, x\rangle+\frac{1}{2}\langle X x, x\rangle+o\left(|x|^{2}\right),
$$

if we choose $\delta=\left(r^{2} / 8\right) \gamma$ then $u(x)>u_{\delta, \gamma}(x)$ for $r / 2 \leq|x| \leq r$ if $r$ is sufficiently small and then, by Lemma 4.2 , the function

$$
U(x)= \begin{cases}\max \left\{u(x), u_{\delta, \gamma}(x)\right\} & \text { if }|x|<r, \\ u(x) & \text { otherwise, }\end{cases}
$$

is a solution of $F \leq 0$ in $\Omega$. The last observation is that in every neighborhood of 0 there are points such that $U(x)>u(x)$; indeed, by definition, there is a sequence $\left(x_{n}, u\left(x_{n}\right)\right)$ convergent to $\left(0, u_{*}(0)\right)$ and then

$$
\lim _{n \rightarrow \infty}\left(U\left(x_{n}\right)-u\left(x_{n}\right)\right)=u_{\delta, \gamma}(0)-u_{*}(0)=u_{*}(0)+\delta-u_{*}(0)>0 .
$$

We summarize what this "bump" construction provides in the following lemma, the proof of which consists only of choosing $r, \gamma$ sufficiently small.

Lemma 4.4. Let $\Omega$ be open and $u$ be solution of $F \leq 0$ in $\Omega$. If $u_{*}$ fails to be a supersolution at some point $\hat{x}$, i.e., there exists $(p, X) \in J_{\Omega}^{2,-} u_{*}(\hat{x})$ for which $F\left(\hat{x}, u_{*}(\hat{x}), p, X\right)<0$, then for any small $\kappa>0$ there is a subsolution $U_{\kappa}$ of $F \leq 0$ in $\Omega$ satisfying

$$
\left\{\begin{array}{l}
U_{\kappa}(x) \geq u(x) \text { and } \sup _{\Omega}\left(U_{\kappa}-u\right)>0, \\
U_{\kappa}(x)=u(x) \text { for } x \in \Omega,|x-\hat{x}| \geq \kappa .
\end{array}\right.
$$

Proof of Theorem 4.1. With the notation of the theorem observe that $\underline{u}_{*} \leq$ $W_{*} \leq W \leq W^{*} \leq \bar{u}^{*}$ and, in particular, $W_{*}=W=W^{*}=0$ on $\partial \Omega$. By Lemma $4.2 W^{*}$ is a subsolution of (DP) and hence, by comparison, $W^{*} \leq \bar{u}$. It then follows from the definition of $W$ that $W=W^{*}$ (so $W$ is a subsolution). If $W_{*}$ fails to be a supersolution at some point $\hat{x} \in \Omega$, let $W_{\kappa}$ be provided by Lemma 4.4. Clearly $\underline{u} \leq W_{\kappa}$ and $W_{\kappa}=0$ on $\partial \Omega$ for sufficiently small $\kappa$. By comparison, $W_{\kappa} \leq \bar{u}$ and since $W$ is the maximal subsolution between $\underline{u}$ and $\bar{u}$, we arrive at the contradiction $W_{\kappa} \leq W$. Hence $W_{*}$ is a supersolution of (DP) and then, by comparison for (DP), $W^{*}=W \leq W_{*}$, showing that $W$ is continuous and is a solution.

Remarks 4.5. The assumption that $\Omega$ was open in Lemma 4.4 was used only to know that classical subsolutions in small relative neighborhoods of points of $\Omega$ were subsolutions in our sense. In order to generalize this and to formulate the version of Theorem 4.1 we will need in $\S 7$ (which we did not do above for pedagogical reasons), we now make some remarks and invite the reader to ignore them until they are called for later. Suppose $\mathscr{O}$ is locally compact, $G^{+}$, $G_{-}$are defined on $\mathscr{O} \times \mathbb{R} \times \mathbb{R}^{N} \times \mathscr{S}(N)$ and have the following properties: $G^{+}$ is upper semicontinous, $G_{-}$is lower semicontinuous, and classical solutions (twice continuously differentiable solutions in the pointwise sense) of $G_{+} \leq 0$ on relatively open subsets of $\mathscr{\theta}$ are solutions of $G_{-} \leq 0$. Suppose, moreover, that whenever $u$ is a solution of $G_{-} \leq 0$ on $\mathscr{O}$ and $v$ is a solution of $G^{+} \geq 0$ on $\mathscr{O}$ we have $u \leq v$ on $\mathscr{O}$. Then we conclude that the existence of such a subsolution and supersolution guarantees that there is a unique function $u$, obtained by the Perron construction, that is a solution of both $G_{+} \geq 0$ and $G_{-} \leq 0$ on $\mathscr{O}$. The proof is unchanged except in trivial ways. 
Example 4.6. Theorem 4.1 leaves open the question of when a subsolution $\underline{u}$ and a supersolution $\bar{u}$ of (DP) that vanish on $\partial \Omega$ can be found. Let us consider this problem for the equation

$$
\left\{\begin{aligned}
u+G\left(x, D u, D^{2} u\right) & =0 & & \text { in } \Omega, \\
u & =0 & & \text { on } \partial \Omega
\end{aligned}\right.
$$

where $G$ is degenerate elliptic. Sometimes there may be an obvious choice for $\underline{u}$ or $\bar{u}$; e.g., if

$$
G(x, 0,0) \leq 0 \text { for } x \in \Omega,
$$

then $\underline{u} \equiv 0$ is a subsolution. We assume (4.10) and seek a supersolution "near $\partial \Omega$ " in the form

$$
u_{1}(x)=M\left(1-e^{-\lambda d(x)}\right)
$$

where $M, \lambda>0$ are parameters to be chosen later and

$$
d(x)=\inf _{y \in \partial \Omega}|y-x|
$$

is the distance to $\partial \Omega$. For $\gamma>0$ set

$$
\Omega_{\gamma}=\left\{x \in \Omega: d(x)<\frac{1}{\gamma}\right\} ;
$$

if $\partial \Omega$ is of class $C^{2}$ and $\gamma$ is large, then $d \in C^{2}\left(\bar{\Omega}_{\gamma}\right)$. We have

$$
\begin{aligned}
u_{1}+G\left(x, D u_{1}(x), D^{2} u_{1}(x)\right) \\
\quad \geq G\left(x, \lambda M e^{-\lambda d(x)} D d(x), \lambda M e^{-\lambda d(x)} D^{2} d(x)-\lambda^{2} M e^{-\lambda d(x)} D d(x) \otimes D d(x)\right)
\end{aligned}
$$

in $\Omega_{\gamma}$, where $p \otimes q$ is the matrix with entries $p_{i} q_{j}$. If $x \in \Omega_{\lambda}$, then $\lambda d(x) \leq 1$ and so

$$
e^{-1} \leq e^{-\lambda d(x)} \leq 1 \quad \text { in } \Omega_{\lambda} .
$$

Choose $M>0$ so that

$$
M\left(1-e^{-1}\right)+G(x, 0,0)>1 \text { in } \Omega .
$$

Then we assume that there is a large $\lambda>0$ so that

$$
\begin{gathered}
G\left(x, \lambda c D d(x), \lambda c D^{2} d(x)-\lambda^{2} c D d(x) \otimes D d(x)\right) \geq 0 \\
\text { for } x \in \Omega_{\lambda}, M e^{-1} \leq c \leq M .
\end{gathered}
$$

Then, by assumption, $u_{1}$ is a classical solution of $u+G\left(x, D u, D^{2} u\right) \geq 0$ in $\Omega_{\lambda}$ and $u_{1}=0$ holds (continuously) on $\partial \Omega$.

To complete the construction, choose $C>0$ so that

$$
C+G(x, 0,0) \geq 0 \text { in } \Omega
$$

and

$$
0<C<M\left(1-e^{-1}\right) ;
$$

this is possible in view of (4.13). We claim that if $\bar{u}$ is defined by

$$
\bar{u}= \begin{cases}u_{1} \wedge C & \text { in } \bar{\Omega}_{\lambda}, \\ C & \text { in } \Omega \backslash \bar{\Omega}_{\lambda},\end{cases}
$$


then it is a supersolution as desired. Indeed, $\bar{u}=0$ on $\partial \Omega$ is evident. The constant $C$ is a supersolution of the equation in $\Omega$ by (4.15) and the subset of $\bar{\Omega}_{\lambda}$ on which $u_{1}=C$ does not meet $\partial\left(\Omega_{\lambda}\right)$ (because of (4.16) and $u_{1}=0$ on $\partial \Omega$ and $u_{1}=M\left(1-e^{-1}\right)$ when $\left.\lambda d(x)=1\right)$. Since the property of being a supersolution is local and closed under finite minimums, we are done.

We have left the sufficient condition for our construction, (4.14), in a "raw form." It is not useful to refine it further without specializing $G$ further. For example, if $G$ has the form $G(x, p, X)=G_{0}(p, X)-f(x)$ where $f \in C(\bar{\Omega})$, in which the $x$-dependence is "separated," the condition that

$$
\lim _{\lambda \rightarrow \infty} G_{0}\left(\lambda c D d(x), \lambda c D^{2} d(x)-\lambda^{2} c D d(x) \otimes D d(x)\right)=\infty
$$

uniformly in $c$ in each interval $\varepsilon \leq c \leq 1 / \varepsilon, \varepsilon>0$, and $x$ in some neighborhood of $\partial \Omega$ in $\bar{\Omega}$ will certainly allow us to satisfy (4.14). In particular, if $G_{0}$ depends only on $p$,

$$
\lim _{|p| \rightarrow \infty} G_{0}(p)=\infty
$$

and $G_{0}(0)-f(x) \leq 0$, we can uniquely solve the Dirichlet problem for $u+$ $G_{0}(D u)-f(x)=0$. Here we rely on the fact that $|D d(x)|=1$ at points of differentiability of $d$; near $\partial \Omega$ (which is all we need) this follows from the obvious fact that $D d(x)=-n(x)$ on $\partial \Omega$ where $n(x)$ is the exterior normal to $\Omega$ at $x \in \partial \Omega$.

In other cases, second-order terms dominate. If

$$
G(x, p, X)=-\operatorname{trace}(A(x) X)+\langle b(x), p\rangle-f(x)
$$

where $A, b, f$ are continuous, and

$$
\langle A(x) n(x), n(x)\rangle>0 \text { on } \partial \Omega,
$$

then the left-hand side of (4.14) has the form

$$
c \lambda^{2}\langle A(x) D d(x), D d(x)\rangle+O(\lambda) \quad \text { as } \lambda \rightarrow \infty .
$$

Since $D d(x)=-n(x)$ on $\partial \Omega$, the coefficient of $\lambda^{2}$ is positive near $\partial \Omega$ and it is easy to achieve (4.14) by taking $\lambda$ large. Note that we are thus able to assert the unique existence of a solution to (4.9) in this case (provided comparison holds) even though $A$ may be completely degenerate inside $\Omega$.

The reader can invent an unlimited array of examples. It should be noted that one may often produce both a subsolution and a supersolution by constructions like the above and then one need not assume $u \equiv 0$ is a subsolution. One may also usually take maximums and minimums of operators for which (4.14) can be verified and stay within this class. Thus, for example, if

$$
G(x, p, X)=\max \left\{-|p|^{2 \theta-\varepsilon}-|\operatorname{trace} X|^{\theta-1} \operatorname{trace} X-f(x),|p|^{\alpha}-g(x)\right\}
$$

and $0<\varepsilon \leq 2 \theta, \alpha>0, f, g \in C(\bar{\Omega})$, and $f, g \geq 0$, then (4.9) has a unique solution.

Notes on $\S 4$. The combination of Perron's method and viscosity solutions was introduced by $\mathrm{H}$. Ishii [86]. The definition of a viscosity subsolution (respectively, supersolution) $u$ in [86] was that $u^{*}$ is a subsolution (respectively, $u_{*}$ is a supersolution) in the current sense. Solutions are then functions that are 
both a subsolution and a supersolution and continuity is not required. (Note that then the characteristic function of the rationals is a solution of $u^{\prime}=0$ ). With this setup, Perron's method does not require the comparison assumption and the statements become more elegant (see [86]).

We mention some other approaches to existence, for even if they are in general much more complicated and of a more limited scope, they can be useful in some delicate situations. For example, one can use formulas from control and differential games to write explicit solutions for approximate equations and then use limiting arguments; this approach is used in M. G. Crandall and P. L. Lions [55, Parts III and V] and D. Tataru [157].

Two other approximations that have been used are discretization and elliptic regularization (for first-order equations; this is the method of "vanishing viscosity" and its relation to the theory accounts for the term "viscosity solutions"). Having solved an approximate problem, one then needs to pass to the limit (with some a priori estimates-but see $\S 6$ !). Existence schemes of this sort have been used in P. L. Lions [116], M. G. Crandall and P. L. Lions [53], G. Barles $[14,15]$, H. Ishii $[82,85]$, P. E. Souganidis [154], and I. Capuzzo-Dolcetta and P. L. Lions [44].

\section{COMPARISON AND PERRON'S Method: VARIATIONS}

5.A. Comparison with more regularity. Suppose that the supersolution $u$ in Theorem 3.3 is more regular; for example, $u \in C^{\gamma}(\Omega)$ where $C^{\gamma}(\Omega)$ denotes the set of functions that are Hölder continuous with exponent $\gamma \in(0,1]$ on each compact subset of $\Omega$. Then the assertions of Theorem 3.3 remain valid if $(3.14)$ is weakened to

$$
\left\{\begin{array}{l}
F(y, r, \alpha(x-y), Y)-F(x, r, \alpha(x-y), X) \leq \omega\left(\alpha|x-y|^{\theta}+|x-y|\right) \\
\text { whenever } x, y \in \bar{\Omega}, r \in \mathbb{R}, X, Y \in \mathscr{S}(N), \text { and (3.10) holds }
\end{array}\right.
$$

for some $\theta>2-\gamma$. Indeed, in the notation of the proof of Theorem 3.3,

$$
u(x)-v(y)-\frac{\alpha}{2}|x-y|^{2} \leq u(\hat{x})-v(\hat{y})-\frac{\alpha}{2}|\hat{x}-\hat{y}|^{2}
$$

implies, upon putting $x=y=\hat{y}$ and recalling that $\hat{x}$ will remain in a compact subset of $\Omega$ as $\alpha \rightarrow \infty, \alpha|\hat{x}-\hat{y}|^{2} \leq 2(u(\hat{x})-u(\hat{y})) \leq C|\hat{x}-\hat{y}|^{\gamma}$ and then $\alpha|\hat{x}-\hat{y}|^{\theta} \rightarrow 0$ as $\alpha \rightarrow \infty$ provided only $\theta>2-\gamma$, so the rest of the proof is unchanged.

More generally and precisely, if $u$ is uniformly continuous with the modulus $\rho / 2$, i.e., $|u(x)-u(y)| \leq \rho(|x-y|) / 2$, we have $\alpha|\hat{x}-\hat{y}|^{2}-\rho(|\hat{x}-\hat{y}|) \leq 0$. Defining $h(\alpha)=\sup \left\{r: \alpha r^{2}-\rho(r) \leq 0\right\}$, we will succeed if $\omega\left(\alpha|x-y|^{\theta}+|x-y|\right)$ in (5.1) is replaced by $\omega(\alpha k(|x-y|)+|x-y|)$ where $\alpha k(h(\alpha)) \rightarrow 0$ as $\alpha \rightarrow$ $\infty$. Since the best possible modulus for nonconstant functions behaves like $\rho(r)=c r$, in which case $h(\alpha)=c / \alpha$ and $k(r)=o(r)$ succeeds, the Lipschitz continuous case is limiting with respect to these arguments (however, see below for the $C^{1}$ case).

If we assume still more regularity of $u$, e.g., $D u \in C^{\gamma}(\Omega)$ where $0 \leq \gamma \leq 1$ (with $\gamma=0$ meaning continuity and $\gamma=1$ meaning Lipschitz continuity of $D u$ ), we need to adapt our strategy to obtain further results. 
Let $K \subset \Omega$ be a compact neighborhood of the set of maximum points of $u-v$ in $\Omega$. If $D u \in C^{\gamma}$ there exists a sequence $\psi_{n}$ in $C(\bar{\Omega}) \cap C^{2}(K)$ with the properties

$$
u-\psi_{n} \rightarrow 0 \text { as } n \rightarrow \infty \text { uniformly on } \Omega
$$

and

$$
\begin{cases}\left|D u-D \psi_{n}\right| \leq C n^{-\gamma} & \text { on } K, \\ \left|D^{2} \psi_{n}\right| \leq C n^{1-\gamma} & \text { on } K, \\ \left|D^{2} \psi_{n}(x)-D^{2} \psi_{n}(y)\right| \leq C n^{2-\gamma}|x-y| & \text { for } x, y \in K\end{cases}
$$

as $n \rightarrow \infty$ where $C$ is a constant. The $\psi_{n}$ may then be constructed by mollification of $u$ near $K$ and then extended.

Let $(\hat{x}, \hat{y})$ be a maximum point of

$$
\left(u(x)-\psi_{n}(x)\right)-\left(v(y)-\psi_{n}(y)\right)-\frac{\alpha}{2}|x-y|^{2} ;
$$

as before, we may assume that $\hat{x}, \hat{y} \in K$ for large $\alpha$ and $n$. Since $D u(\hat{x})-$ $D \psi_{n}(\hat{x})=\alpha(\hat{x}-\hat{y})$ and (5.3) holds, we have

$$
\alpha|\hat{x}-\hat{y}| \leq C n^{-\gamma} \text { as } n \rightarrow \infty .
$$

Modifying the arguments in the proof of Theorem 3.3 in an obvious way, we will still be able to establish comparison if we can show that

$$
\begin{aligned}
& \left(F\left(\hat{y}, r, \alpha(\hat{x}-\hat{y})+D \psi_{n}(\hat{y}), Y+D^{2} \psi_{n}(\hat{y})\right)\right. \\
& \left.\quad-F\left(\hat{x}, r, \alpha(\hat{x}-\hat{y})+D \psi_{n}(\hat{x}), X+D^{2} \psi_{n}(\hat{x})\right)\right)^{+} \rightarrow 0
\end{aligned}
$$

as $n, \alpha \rightarrow \infty$ in some appropriate fashion when $r$ remains bounded and (3.10), (5.3), (5.5) hold.

It is not very informative to try and analyze this condition in general. Instead, let us note that in the linear case (see Remark 3.6)

$$
F(x, r, p, X)=-\operatorname{trace}\left({ }^{t} \Sigma(x) \Sigma(x) X\right)+\langle b(x), p\rangle+r-f(x)
$$

with $f \in C(\Omega)$, the first-order terms are harmless from the point of view of verifying (5.6) if $b$ is continuous. Via Remark 3.6, the second-order terms contribute at most

$$
3 \alpha \operatorname{trace}\left({ }^{t}(\Sigma(\hat{x})-\Sigma(\hat{y}))(\Sigma(\hat{x})-\Sigma(\hat{y}))+\operatorname{trace}\left(-A(\hat{y}) D^{2} \psi_{n}(\hat{y})+A(\hat{x}) D^{2} \psi_{n}(\hat{x})\right)\right.
$$

when estimating (5.6) above. Here we have put $A(x)={ }^{t} \Sigma(x) \Sigma(x)$. Assuming $\Sigma \in C^{\lambda}(\Omega)$ (so $A \in C^{\lambda}(\Omega)$ ), we invoke (5.3) and estimate the above expression by a constant times

$$
\alpha|\hat{x}-\hat{y}|^{2 \lambda}+n^{1-\gamma}|\hat{x}-\hat{y}|^{\lambda}+n^{2-\gamma}|\hat{x}-\hat{y}| .
$$

By (5.5), this expression may, in turn, be estimated above in the form

$$
\left(\alpha\left(\frac{n^{-\gamma}}{\alpha}\right)^{2 \lambda}+n^{1-\gamma}\left(\frac{n^{-\gamma}}{\alpha}\right)^{\lambda}+n^{2-\gamma}\left(\frac{n^{-\gamma}}{\alpha}\right)\right) \quad \text { as } n \rightarrow \infty .
$$

Putting $\alpha=n^{\beta}$, this becomes

$$
\left(n^{\beta-2 \lambda(\gamma+\beta)}+n^{-\lambda(\gamma+\beta)+1-\gamma}+n^{2(1-\gamma)-\beta}\right) \quad \text { as } n \rightarrow \infty .
$$


Regarding $\lambda$ as fixed so as to see what we require of $\gamma$, we note that we may make all of the exponents above vanish by the choices $\gamma=(1-2 \lambda) /(1-\lambda)$, $\beta=2 \lambda /(1-\lambda)$. (The case $\lambda>\frac{1}{2}$ was treated above, so here we are concerned with $\lambda \leq \frac{1}{2}$.) If we increase $\gamma$ from this value, so that $\gamma>(1-2 \lambda) /(1-\lambda)$, it follows that comparison holds.

Comparison is thus assured if $\Sigma \in C^{\lambda}$ and $D u \in C^{\gamma}$ under the relation $\gamma>(1-2 \lambda) /(1-\lambda)$. The limit cases $\lambda=\frac{1}{2}$ and $\lambda=0$ need to be discussed separately, for $\gamma=0$ suffices if $\lambda=\frac{1}{2}$ and $\gamma=1$ suffices if $\lambda=0$. In the event that $\gamma=0$, the first relation in (5.3) should be replaced by $\left|u-\psi_{n}\right| \leq o$ (1) and (5.5) by $\alpha|\hat{x}-\hat{y}| \leq o(1)$ and then the proof runs as before for $\gamma=0$ but with the constant $o(1)$ appearing everywhere. To treat the other limiting case, $\lambda=0, \gamma=1$, note that if $\rho$ is a modulus of continuity of $\Sigma$ and $A,(5.7)$ should be replaced by

$$
\alpha \rho(|\hat{x}-\hat{y}|)^{2}+\rho(|\hat{x}-\hat{y}|)+n|\hat{x}-\hat{y}|
$$

and (5.5) should read $\alpha|\hat{x}-\hat{y}| \leq C / n$. We conclude upon letting $n, \alpha \rightarrow \infty$ in a manner so that $\alpha \rho(C /(n \alpha))^{2} \rightarrow 0$.

It is worthwhile to compare the uniqueness results above with those that may be obtained when solutions are more nearly classical in the sense that they possess second derivatives in a strong enough sense. To simplify the exposition, we will always assume that (3.13) holds. Then, of course, if we assume that one of $u$ or $v$ is $C^{2}$ (or even everywhere twice differentiable), the comparison result holds without other structure conditions on $F$ beyond properness, since we can work directly with maxima of $u-v$. Unfortunately, this regularity is rarely available, even for uniformly elliptic fully nonlinear equations. If we require less regularity of $u$ and $v$-but still much more than assumed in Theorem 3.3-different structure conditions suffice to guarantee the comparison result via finer considerations about the pointwise twice differentiability of functions.

We present below a strategy that establishes comparison without further assumptions on $F$ if both $u+c|x|^{2}$ and $-v+c|x|^{2}$ are convex for some $c>0$. It also shows that comparison holds if $u, v \in W_{\mathrm{loc}}^{2, N}(\Omega)$ and one of $u$ or $v$ lies in $W_{\text {loc }}^{2, p}(\Omega)$ for some $p>N$ and the structure condition

$$
\left\{\begin{array}{l}
F(x, t, p, X) \text { is uniformly continuous in } p \\
\text { uniformly for } x \in \bar{\Omega}, X \in \mathscr{S}(N), \text { and } r, p \text { bounded }
\end{array}\right.
$$

holds.

In both of these cases, the argument runs as follows: we may assume, without loss of generality, that all maxima of $u-v$ lie in $\Omega$ and then general optimization results imply that there exists a sequence $p_{n} \rightarrow 0$ such that $u(x)-v(x)-\left\langle p_{n}, x\right\rangle$ has a strict maximum at $\hat{x}_{n}$ and $\hat{x}_{n} \rightarrow \hat{x}$ where $\hat{x}$ is a maximum point of $u-v$ over $\bar{\Omega}$. Then one knows (cf. Lemma A.3) that if $r>0$ is small enough, the set of maximum points of $u(x)-v(x)-\left\langle p_{n}, x\right\rangle-\langle q, x\rangle$ in $\left|x-\hat{x}_{n}\right|<r$ as $q$ ranges over the ball $B_{\delta}$ contains a set of positive measure if $\delta<r^{-1} \varepsilon_{n}$ where

$$
\varepsilon_{n}=\inf _{\left|y-\hat{x}_{n}\right|=r}\left[\left(u\left(\hat{x}_{n}\right)-v\left(\hat{x}_{n}\right)\right)-\left\langle p_{n}, \hat{x}_{n}-y\right\rangle-(u(y)-v(y))\right] .
$$

Furthermore, either $u$ or $v$ is twice differentiable a.e. (cf. Theorem A.2) and therefore we can find maxima $z$ with $\left|\hat{x}_{n}-z\right|<r$ for some $q \in B_{\delta}$ that is 
a point where, in the first case, $u$ and $v$ are twice differentiable and in the second case $v$ or $u$ is twice differentiable. In the first case we have

$$
F\left(z, u(z), D v(z)+p_{n}+q, D^{2} v(z)\right) \leq 0 \leq F\left(z, v(z), D v(z), D^{2} v(z)\right) \text {. }
$$

In the second case, if $v$ is twice differentiable the same inequality holds and otherwise we have

$$
F\left(z, u(z), D u(z), D^{2} u(z)\right) \leq 0 \leq F\left(z, v(z), D u(z)-p_{n}-q, D^{2} u(z)\right) .
$$

The conclusions are easily reached upon sending $\delta, r$ to $0+$ and then $n \rightarrow \infty$.

5.B. Estimates from comparison. We make some simple remarks that hold as soon as one has comparison by any means; there are many variants of these (including parabolic ones). For example, suppose $K>0$ and $u-v \leq K$ on $\partial \Omega$ instead of $u \leq v$ on $\partial \Omega$. Then $u-K$ is also a subsolution since $J_{\Omega}^{2,+}(u-K)=J_{\Omega}^{2,+} u$ and $F(x, u-K, p, X) \leq F(x, u, p, X)$ by properness. Thus $u-v \leq K$ in $\bar{\Omega}$. In particular, the variant of Theorem 3.3 in which $u \leq v$ on $\partial \Omega$ is dropped and the conclusion is changed to $u-v \leq \sup _{\partial \Omega}(u-v)^{+}$ holds.

In a similar spirit, suppose we have solutions $u$ and $v$ of $F\left(x, u, D u, D^{2} u\right)$ $\leq 0$, and $\hat{F}\left(x, u, D u, D^{2} u\right) \geq 0$ in $\Omega$ where $F$ is proper, satisfies (3.14) and (3.13), $K>0$, and $F(x, r, p, X)+K \geq \hat{F}(x, r, p, X)$. Then $w=$ $v+\max \left(\sup _{\partial \Omega}(u-v)^{+}, K / \gamma\right)$ is a solution of $F\left(x, w, D w, D^{2} w\right) \geq 0$ in $\Omega$. Since we have comparison for $F$, we then conclude $u-w \leq \sup _{\partial \Omega}(u-w)^{+}=0$ or $u-v \leq \max \left(\sup _{\partial \Omega}(u-v)^{+}, K / \gamma\right)$. In particular, if

$u+G\left(x, u, D u, D^{2} u\right)-f(x) \leq 0$ and $v+G\left(x, v, D v, D^{2} v\right)-g(x) \geq 0$ in $\Omega$, $G$ is proper and satisfies (3.14), and $f, g \in C(\bar{\Omega})$, then

$$
u-v \leq \max \left(\sup _{\partial \boldsymbol{\Omega}}(u-v)^{+}, \sup _{\Omega}(f-g)^{+}\right) \text {. }
$$

5.C. Comparison with strict inequalities and without coercivity in $u$. The condition (3.13) was used in the proof of Theorem 3.3 in order to have (3.15). If we simply assume there is a $\delta>0$ such that either $F\left(x, u, D u, D^{2} u\right) \leq-\delta$ or $F\left(x, v, D v, D^{2} v\right) \geq \delta$ we are in the same situation and do not need (3.13) to hold. Moreover, if only $F\left(x, u, D u, D^{2} u\right) \leq 0$ and $F\left(x, v, D v, D^{2} v\right)$ $\geq 0$ but for $\varepsilon>0$ we can find $\psi_{\varepsilon} \in C^{2}, \delta_{\varepsilon}>0$ such that $\left|\psi_{\varepsilon}\right| \leq \varepsilon$ and $F\left(x, u+\psi_{\varepsilon}, D\left(u+\psi_{\varepsilon}\right), D^{2}\left(u+\psi_{\varepsilon}\right)\right) \leq-\delta_{\varepsilon}$, we conclude that $\left(u+\psi_{\varepsilon}\right)-v \leq$ $\sup _{\partial \Omega}\left(u+\psi_{\varepsilon}-v\right)$ and then $u-v \leq \sup _{\partial \Omega}(u-v)+2 \varepsilon$ and we recover the result as $\varepsilon \downarrow 0$. This construction can be carried out in some cases.

5.D. Comparison and existence of unbounded solutions on unbounded domains. We first illustrate a method to establish comparison of unbounded solutions in unbounded domains by showing that if $u$ and $v$ grow at most linearly and solve

$$
u+F\left(D u, D^{2} u\right)-f(x) \leq 0 \text { and } v+F\left(D v, D^{2} v\right)-f(x) \geq 0 \text { in } \mathbb{R}^{N}
$$

where $f$ is uniformly continuous on $\mathbb{R}^{N}$ (i.e., $f \in \operatorname{UC}\left(\mathbb{R}^{N}\right)$ ), then $u \leq v$. After this, we will prove that if $u$ is a solution of $u+F\left(D u, D^{2} u\right)=\overline{0}$ of linear growth, then $u \in \mathrm{UC}\left(\mathbb{R}^{N}\right)$ and finally that Perron's method supplies existence. Thus we will prove 
Theorem 5.1. If $f \in \mathrm{UC}\left(\mathbb{R}^{N}\right)$, then $u+F\left(D u, D^{2} u\right)-f(x)=0$ has a unique solution $u$ that grows at most linearly and $u \in \mathrm{UC}\left(\mathbb{R}^{N}\right)$.

Proof of comparison. The proof proceeds in two steps. First we note that $f \in$ $\mathrm{UC}\left(\mathbb{R}^{N}\right)$ implies that there is a constant $K$ such that

$$
\sup _{\mathbf{R}^{N} \times \mathbf{R}^{N}}(f(x)-f(y)-K|x-y|)<\infty
$$

and then we show that

$$
\sup _{\mathbf{R}^{N} \times \mathbb{R}^{N}}(u(x)-v(y)-2 K|x-y|)<\infty .
$$

By the assumed linear growth, we have

$$
u(x)-v(y) \leq L(1+|x|+|y|) \text { on } \mathbb{R}^{N} \times \mathbb{R}^{N}
$$

for some $L>0$. We choose a family $\beta_{R}$ of $C^{2}$ functions on $\mathbb{R}^{N}$ parameterized by $R \geq 1$ with the properties

$$
\left\{\begin{array}{l}
\text { (i) } \beta_{R} \geq 0, \\
\text { (ii) } \liminf _{|x| \rightarrow \infty} \beta_{R}(x) /|x| \geq 2 L, \\
\text { (iii) }\left|D \beta_{R}(x)\right|+\left\|D^{2} \beta_{R}(x)\right\| \leq C \text { for } R \geq 1, x \in \mathbb{R}^{N}, \\
\text { (iv) } \lim _{R \rightarrow \infty} \beta_{R}(x)=0 \text { for } x \in \mathbb{R}^{N},
\end{array}\right.
$$

where $C$ is some constant. In view of (5.11) and (5.12)(ii), the function

$$
\Phi(x, y)=u(x)-v(y)-2 K\left(1+|x-y|^{2}\right)^{1 / 2}-\left(\beta_{R}(x)+\beta_{R}(y)\right)
$$

attains its maximum at some point $(\hat{x}, \hat{y})$. Now either $(5.10)$ holds or for large $R$ we have $\Phi(\hat{x}, \hat{y})>0$, which implies

$$
2 K|\hat{x}-\hat{y}| \leq u(\hat{x})-v(\hat{y}) .
$$

Noting that

$$
\begin{gathered}
\left(\hat{p}+D \beta_{R}(\hat{x}), \hat{Z}+D^{2} \beta_{R}(\hat{x})\right) \in J^{2,+} u(\hat{x}), \\
\left(\hat{p}-D \beta_{R}(\hat{y}),-\hat{Z}-D^{2} \beta_{R}(\hat{y})\right) \in J^{2,-} v(\hat{y}),
\end{gathered}
$$

where

$$
\hat{p}=\left.\left(2 K D_{z}\left(1+|z|^{2}\right)^{1 / 2}\right)\right|_{z=\hat{x}-\hat{y}}, \quad \hat{Z}=\left.\left(2 K D_{z}^{2}\left(1+|z|^{2}\right)^{1 / 2}\right)\right|_{z=\hat{x}-\hat{y}},
$$

we see that

$$
\begin{aligned}
& u(\hat{x})+F\left(\hat{p}+D \beta_{R}(\hat{x}), \hat{z}+D^{2} \beta_{R}(\hat{x})\right) \leq f(\hat{x}), \\
& v(\hat{y})+F\left(\hat{p}-D \beta_{R}(\hat{y}),-\hat{z}-D^{2} \beta_{R}(\hat{y})\right) \geq f(\hat{y}) .
\end{aligned}
$$

From this, using (5.13) and observing that $\hat{p}$ and $\hat{Z}$ are bounded independently of $R \geq 1$, we have

$$
\begin{aligned}
u(\hat{x})-v(\hat{y}) \leq & f(\hat{x})-f(\hat{y})+F\left(\hat{p}-D \beta_{R}(\hat{y}),-\hat{Z}-D^{2} \beta_{R}(\hat{y})\right) \\
& -F\left(\hat{p}+D \beta_{R}(\hat{x}), \hat{z}+D^{2} \beta_{R}(\hat{x})\right) \\
\leq & K|\hat{x}-\hat{y}|+C \leq \frac{1}{2}(u(\hat{x})-v(\hat{y}))+C
\end{aligned}
$$

where $C$ is a constant independent of $R \geq 1$. Therefore, $u(\hat{x})-v(\hat{y})$ is bounded independently of $R \geq 1$. 
Since $\Phi(x, y) \leq \Phi(\hat{x}, \hat{y}) \leq u(\hat{x})-v(\hat{y})$, we may then send $R \rightarrow \infty$ to conclude that $u(x)-v(y)-2 K\left(1+|x-y|^{2}\right)^{1 / 2}$ is bounded and thus that (5.10) holds. With the information (5.10) in hand, we repeat the above line of argument, this time assuming $u(\bar{x})-v(\bar{x})=2 \delta>0$ holds for some $\bar{x}$ and putting

$$
\Phi(x, y)=u(x)-v(y)-\frac{\alpha}{2}|x-y|^{2}-\varepsilon\left(|x|^{2}+|y|^{2}\right)
$$

where $\varepsilon, \alpha$ are positive parameters. For small $\varepsilon$ we have $\Phi(\bar{x}, \bar{x}) \geq \delta$ and by $(5.10) \Phi$ will have a maximum $(\hat{x}, \hat{y})$ at which

$$
\frac{\alpha}{2}|\hat{x}-\hat{y}|^{2}+\varepsilon\left(|\hat{x}|^{2}+|\hat{y}|^{2}\right) \leq u(\hat{x})-v(\hat{y}) \leq 2 K|\hat{x}-\hat{y}|+C \leq \frac{\alpha}{4}|\hat{x}-\hat{y}|^{2}+4 K^{2} / \alpha+C
$$

for some $C$. Moreover, there are $X, Y \in \mathscr{S}(N)$ such that

$$
(\alpha(\hat{x}-\hat{y})+2 \varepsilon \hat{x}, X+2 \varepsilon I) \in \bar{J}^{2,+} u(\hat{x}), \quad(\alpha(\hat{x}-\hat{y})-2 \varepsilon \hat{y}, Y-2 \varepsilon I) \in \bar{J}^{2,-} v(\hat{y})
$$

and

$$
-3 \alpha\left(\begin{array}{cc}
I & 0 \\
0 & I
\end{array}\right) \leq\left(\begin{array}{cc}
X & 0 \\
0 & -Y
\end{array}\right) \leq 3 \alpha\left(\begin{array}{cc}
I & -I \\
-I & I
\end{array}\right) .
$$

As usual, we have

$$
\begin{aligned}
u(\hat{x})-v(\hat{y}) \leq & f(\hat{x})-f(\hat{y})+F(\alpha(\hat{x}-\hat{y})-2 \varepsilon \hat{y}, Y-2 \varepsilon I) \\
& -F((\alpha(\hat{x}-\hat{y})+2 \varepsilon \hat{x}, X+2 \varepsilon I) .
\end{aligned}
$$

Since $\delta \leq \Phi(\bar{x}, \bar{x}) \leq u(\hat{x})-v(\hat{y})$, and since $X \leq Y$ by $(5.17)$, $\delta \leq \omega_{f}(|\hat{x}-\hat{y}|)+F(\alpha(\hat{x}-\hat{y})-2 \varepsilon \hat{y}, X-2 \varepsilon I)-F(\alpha(\hat{x}-\hat{y})+2 \varepsilon \hat{x}, X+2 \varepsilon I)$, where $\omega_{f}$ is the modulus of continuity of $f$, and we will obtain a contradiction if we show that the iterated limit inferior of the right-hand side as $\varepsilon \downarrow 0$ and then $\alpha \rightarrow \infty$ is nonpositive. This information is deduced from (5.16), first using the extreme inequalities, which imply that $\alpha|\hat{x}-\hat{y}|^{2}$ and $\varepsilon\left(|\hat{x}|^{2}+|\hat{y}|^{2}\right)$ are bounded independently of $\alpha \geq 1$ and $0<\varepsilon \leq 1$. Thus $\varepsilon \hat{x}, \varepsilon \hat{y} \rightarrow 0$ and $\alpha(\hat{x}-\hat{y})$ remains bounded as $\varepsilon \downarrow 0$, while $|\hat{x}-\hat{y}| \rightarrow 0$ as $\alpha \rightarrow \infty$ uniformly in $\varepsilon>0$. Therefore, using (5.17) and the uniform continuity of $F$ on bounded sets,

$$
\delta \leq \liminf _{\alpha \rightarrow \infty} \liminf _{\varepsilon \downarrow 0} \omega_{f}(|\hat{x}-\hat{y}|)=0,
$$

which is a contradiction.

Proof that solutions lie in $\mathrm{UC}\left(\mathbb{R}^{N}\right)$. We take the easy way here, using that $F$ is independent of $x$, and note that if $y \in \mathbb{R}^{N}$ and $u$ solves $u+F\left(D u, D^{2} u\right)-$ $f(x)=0$, then $w(x)=u(x+y)$ solves $w+F\left(D w, D^{2} w\right)-f(x+y)=0$, so that $|u(x)-u(x+y)| \leq \sup _{z \in \mathbf{R}^{N}}|f(z+y)-f(z)|$ by 5.B; thus $u \in \operatorname{UC}\left(\mathbb{R}^{N}\right)$.

Proof of existence. We need only to produce a linearly growing subsolution and supersolution and invoke Perron's method. Letting $\bar{u}(x)=A+$ $B\left(1+|x|^{2}\right)^{1 / 2}$, we note that $D \bar{u}$ and $D^{2} \bar{u}$ are bounded so long as $B$ is bounded. By (5.9), $|f(x)| \leq C+K|x|$ for some $C$. If we put $B=K$ and $A=$ $\sup _{\mathbf{R}^{N}}\left|F\left(D \bar{u}, D^{2} \bar{u}\right)\right|+C, \bar{u}$ is a supersolution and $\underline{u}=-\bar{u}$ is a subsolution.

Notes on $\S 5$. The discussion of $\S 5$.A regarding regularity of $D u$ is new in this framework, but such results were first obtained by $R$. Jensen [102] in a more 
complicated presentation. The assertions of $\S 5$.A regarding twice differentiable solutions (classical or $W^{2, p}$, etc.) rely on various versions of the maximum principle as it evolved through the works of Aleksandrov [2, 3], Y. Bakelman [6], C. Pucci [143], J. M. Bony [37], and P. L. Lions [120].

Section 5.C recalls a classical strategy and corresponds to remarks used in $\mathbf{M}$. G. Crandall and P. L. Lions [51], H. Ishii [87], H. Ishii and P. L. Lions [96], .... More sophisticated uses of estimates from comparison arguments occur in the study of regularity questions-see $\S 10$ for references.

Section 5.D is concerned with the growth of solutions at infinity and its influence on comparison-uniqueness results. The relevance of the class $U C\left(\mathbb{R}^{N}\right)$ for general results of this sort was progressively understood in a series of papers by the authors and we refer to M. G. Crandall and P. L. Lions [55, Part II] for a few examples showing how natural this class is and how it interacts with structure conditions on the nonlinearity. Of course, if one restricts the nonlinearity further, other asymptotic behaviors can be allowed. See H. Ishii [83, 89], M. G. Crandall and P. L. Lions [54, 56], and M. G. Crandall, R. Newcomb, and $Y$. Tomita [59] for more information in this direction; in particular, general functions $F(x, r, p, X)$ can be used in place of $F(p, X)-f(x)$ (although subtleties concerning existence arise).

\section{LIMIT OPERATIONS WITH VISCOSITY SOLUTIONS}

Suppose we have a sequence $u_{n}, n=1,2, \ldots$, of subsolutions of an equation $F=0$ on the locally compact set $\mathscr{O}$. It turns out that the following "limit"

$$
\bar{U}(z)=\limsup _{n \rightarrow \infty}{ }^{*} u_{n}(z)=\lim _{j \rightarrow \infty} \sup \left\{u_{n}(x): n \geq j, x \in \mathscr{O}, \text { and }|z-x| \leq \frac{1}{j}\right\}
$$

in which, roughly speaking, the "lim sup" operation and the ${ }^{*}$ operations are performed simultaneously, will also be a solution of $F \leq 0$ in $\mathscr{O}$. Indeed, since $\bar{U}(x)<r$ only if there are $\varepsilon, j>0$ such that $u_{n}(z)<r-\varepsilon$ for $n \geq j$ and $z \in \mathscr{O}$ with $|z-x| \leq 1 / j$, it is clear that $\{x \in \mathscr{O}: \bar{U}(x)<r\}$ is open in $\mathscr{O}$ and thus $\bar{U} \in \operatorname{USC}(\mathscr{O})$. We have

Lemma 6.1. Let $u_{n} \in \operatorname{USC}(\mathscr{O})$ for $n=1,2, \ldots$ and $\bar{U}$ be given by (6.1), $z \in \mathscr{O}$ and $\bar{U}(z)<\infty$. If $(p, X) \in J_{\mathscr{Q}}^{2,+} \bar{U}(z)$, then there exist sequences

$$
n_{j} \rightarrow \infty, \quad \hat{x}_{j} \in \mathscr{O}, \quad\left(p_{j}, X_{j}\right) \in J_{\mathscr{O}}^{2,+} u_{n_{j}}\left(\hat{x}_{j}\right)
$$

such that

$$
\left(\hat{x}_{j}, u_{n_{j}}\left(\hat{x}_{j}\right), p_{j}, X_{j}\right) \rightarrow(z, \bar{U}(z), p, X) .
$$

In particular, if each $u_{n}$ is a solution of $F \leq 0$ and $\bar{U}<\infty$ on $\mathscr{O}$, then $\bar{U}$ is a solution of $F \leq 0$ on $\mathscr{O}$.

Proof. By definition, there are sequences

$$
n_{j} \rightarrow \infty, x_{j} \in \mathscr{O} \text { such that } x_{j} \rightarrow z \text { and } u_{n_{j}}\left(x_{j}\right) \rightarrow \bar{U}(z) ;
$$

it is also clear that if $z_{j} \rightarrow x$ in $\mathscr{O}$, then $\lim \sup u_{n_{j}}\left(z_{j}\right) \leq \bar{U}(x)$. The result now follows at once from Proposition 4.3. 
Remark 6.2. If the assumptions of the lemma are altered to assume instead that $u_{n} \in \operatorname{LSC}(\mathscr{O})$ and $(p, X) \in J_{\mathscr{O}}^{2,-} v(z)$, then the assertions are changed by replacing $\bar{U}$ by

$$
\underline{U}(z)=\liminf _{n \rightarrow \infty} u_{n}(z)=\lim _{j \rightarrow \infty} \inf \left\{u_{n}(x): n \geq j, x \in \mathscr{O}, \text { and }|z-x| \leq \frac{1}{j}\right\},
$$

putting $J_{\mathscr{O}}^{2,-}$ in place of $J_{\mathscr{O}}^{2,+}$ in (6.2) and supersolutions in place of subsolutions.

Remark 6.3. In fact, the above proof shows more-suppose $u_{n}$ is a solution of a proper equation $F_{n} \leq 0$ that varies with $n$. Then the conclusion is that $\bar{U}$ is a solution of $G \leq 0$ where

$$
G(x, r, p, X)=\liminf _{n \rightarrow \infty} F_{n}(x, r, p, X) ;
$$

note that $F_{n}$ need not be continuous and that if $F_{n}=F$ is independent of $n$ but discontinuous, then $G=F_{*}$ is the lower semicontinuous envelope of $F$. Analogous remarks hold for supersolutions.

Remark 6.4. The above results are related to uniform convergence as explained next. Let $u_{n}$ be a sequence of functions on $\mathscr{O}$ and

$$
\bar{U}(x)=\limsup _{n \rightarrow \infty} u_{n}(x), \quad \underline{U}(x)=\liminf _{n \rightarrow \infty} u_{n}(x) .
$$

Suppose $\bar{U}(x)=\underline{U}(x)$ on $\mathscr{O}$, let $U(x)$ denote this common value and assume that $-\infty<U(x)<\infty$ on $\mathscr{O}$. Then $U$ is continuous (since it is both upper and lower semicontinuous) and $\lim _{n \rightarrow \infty} u_{n}(x)=U(x)$ uniformly on compact sets. Indeed, if this were not the case and uniform convergence failed on some compact set $K$, there would be an $\varepsilon>0$ and sequences $n_{j} \rightarrow \infty, x_{j} \in K$ such that $u_{n_{j}}\left(x_{j}\right)-U\left(x_{j}\right)>\varepsilon$ or $u_{n_{j}}\left(x_{j}\right)-U\left(x_{j}\right)<-\varepsilon$. Assuming $x_{j} \rightarrow x$ and using the continuity of $U$, we would conclude that $|U(x)-U(x)| \geq \varepsilon$, a contradiction. In order to prove that $\bar{U}=\underline{U}$ one notes that $\underline{U} \leq \bar{U}$ by definition and typically uses comparison results to prove the other inequality. The next result provides a simple example; a typical case in which the hypotheses of this result are easily verified is mentioned after the short proof.

Theorem 6.5. Let $\Omega$ be a bounded open set in $\mathbb{R}^{N}, H \in C\left(\mathbb{R}^{N}\right)$, and $f \in C(\bar{\Omega})$. Consider the problem

$$
u+H(D u)-\varepsilon \Delta u=f(x) \text { in } \Omega, \quad u=0 \text { on } \partial \Omega,
$$

and assume that $(\mathrm{DP})_{\varepsilon}$ has a subsolution $\underline{u} \in C(\bar{\Omega})$ and a supersolution $\bar{u} \epsilon$ $C(\bar{\Omega})$ independent of $\varepsilon \in[0,1]$ that vanish on $\partial \Omega$. Then $(\mathrm{DP})_{\varepsilon}$ has a solution $u_{\varepsilon}$ for $\varepsilon \in[0,1]$ and $\lim _{\varepsilon \downarrow 0} u_{\varepsilon}(x)=u_{0}(x)$ uniformly for $x \in \bar{\Omega}$.

Proof. We know that $(\mathrm{DP})_{\varepsilon}$ has a solution $u_{\varepsilon}$ for $\varepsilon \in[0,1]$ by Perron's method and the assumptions. Moreover, $\bar{U}(x)=\lim \sup _{\varepsilon \downarrow 0}{ }^{*} u_{\varepsilon}(x)$ and $\underline{U}(x)=$ $\lim \inf _{\varepsilon \downarrow 0 *} u_{\varepsilon}(x)$ are a subsolution and a supersolution of (DP) 0 by the above and then $\bar{U} \leq \underline{U}$ by comparison while $\bar{U} \geq \underline{U}$ by definition. Thus $u_{0}=\bar{U}=\underline{U}$ and the convergence is uniform.

Harking back to the discussion of Example 4.6, the reader may easily verify the hypotheses of Theorem 6.5 when $\partial \Omega$ is of class $C^{2}, H$ satisfies 
$\lim _{|p| \rightarrow \infty} H(p)=\infty$ and $H(0)-f \leq 0$. To do so, set $\underline{u} \equiv 0$ and $\bar{u}(x)=$ $(\lambda d(x)) \wedge C$ where $\lambda$ is first chosen sufficiently large and then $C$ is chosen in a suitable manner.

Notes on $\S 6$. The fact that viscosity solutions pass to the limit under uniform convergence is an immediate consequence of the definition. This consistency and stability property can also be seen as the analogue (in the space of continuous functions) of Minty's device for monotone operators as developed by $L$. C. Evans $[64,65]$.

The idea of using only "half-relaxed" limits like $\bar{U}$ in (6.1) arises naturally when attempting to pass to limits with maxima and minima and has been used extensively in the calculus of variations and homogenization theory. The use of this concept in the area of viscosity solutions was introduced by G. Barles and $B$. Perthame $[25,26]$ and $H$. Ishii [90]. This passage to the limit and the associated a posteriori uniform convergence through comparison of semicontinuous functions has become one of the main features of the theory. Viscosity solutions were first used to prove theorems analogous to Theorem 3.2 (but more complicated) with applications by L. C. Evans and H. Ishii [68]; they were motivated by questions arising in probability (see also $\S 10$ ). Proofs in the spirit we have presented are much simpler.

\section{GENERAL AND GENERALIZED BOUNDARY CONDITIONS}

7.A. Boundary conditions in the viscosity sense. In this section we consider more general boundary value problems of the form

$$
\left\{\begin{array}{lll}
(\mathrm{E}) & F\left(x, u, D u, D^{2} u\right)=0 & \text { in } \Omega \\
(\mathrm{BC}) & B\left(x, u, D u, D^{2} u\right)=0 & \text { on } \partial \Omega
\end{array}\right.
$$

where $(\mathrm{E})$ denotes the equation and $(\mathrm{BC})$ the boundary condition. Here $F$ is proper, as always, and $B$ is a given function on $\partial \Omega \times \mathbb{R} \times \mathbb{R}^{N} \times \mathscr{S}(N)$ that is also to be proper. While it is convenient to allow $B$ to depend on $D^{2} u$ at this stage, as the reader will see, when we turn to existence and uniqueness theorems $B=B(x, r, p)$ will be of first order. For example, the Dirichlet condition $u=f(x)$ on $\partial \Omega$ arises from the choice $B(x, r, p, X)=B(x, r, p)=r-f(x)$ of $B$ while the Neumann condition $u_{n}=f(x)$ (here and later, $n(x)$ denotes the outward unit normal to $x \in \partial \Omega)$ arises if $B(x, r, p)=\langle n(x), p\rangle-f(x)$. The Neumann condition is generalized by the "oblique derivative" problem, in which $B$ has the form $B(x, r, p)=\langle\nu(x), p\rangle-f(x)$ where $\nu(x)$ is a vector field on $\partial \Omega$ satisfying $\langle\nu(x), n(x)\rangle>0$ on $\partial \Omega$.

We have already given a meaning to statements like (BC) in Definition 2.2, which is, however, clearly inappropriate here since it would only involve the behavior of $u$ on $\partial \Omega$. The simplest viscosity definition of $(\mathrm{BC})$ we might try is

Definition 7.1. A function $u \in \operatorname{USC}(\bar{\Omega})$ is a subsolution of $(\mathrm{BC})$ in the strong (viscosity) sense if

$$
B(x, u(x), p, X) \leq 0 \text { for } x \in \partial \Omega,(p, X) \in \bar{J}_{\bar{\Omega}}^{2,+} u(x)
$$

and $v \in \operatorname{LSC}(\bar{\Omega})$ is a supersolution of (BC) in the strong (viscosity) sense if

$$
B(x, v(x), p, X) \geq 0 \text { for } x \in \partial \Omega \text {, and }(p, X) \in \bar{J}_{\bar{\Omega}}^{2,-} v(x) \text {. }
$$


Finally, $u \in C(\bar{\Omega})$ satisfies (BC) in the strong (viscosity) sense if it is both a subsolution and a supersolution in the strong sense.

Interpreting (E) in the usual way and (BC) in the strong sense, we obtain notions of subsolutions (which will lie in $\operatorname{USC}(\bar{\Omega})$ ), supersolutions (which will lie in $\operatorname{LSC}(\bar{\Omega})$ ) and solutions (which lie in $C(\bar{\Omega})$ ).

As indicated by the appearance of the modifier "the strong sense," it will be necessary to relax this interpretation to obtain full generality. Before explaining this, however, we pause to relate "the strong sense" to the "classical sense."

It is important to recognize early on that if $u \in C^{2}(\bar{\Omega})$ (i.e., $u$ extends to a twice continuously differentiable function in a neighborhood of $\bar{\Omega}$ ) and

$$
B\left(x, u(x), D u(x), D^{2} u(x)\right) \leq 0 \text { for } x \in \partial \Omega,
$$

then it is not necessarily true that $u$ is a subsolution of $(\mathrm{BC})$. Indeed, in Remark 2.7 we determined $J_{\mathscr{O}}^{2,+} u(x)$ in the situation where $\mathscr{O}$ is an $\mathrm{N}$-submanifold of $\mathbb{R}^{N}$ with boundary, $x \in \partial \Omega$, and $\partial \Omega$ is twice differentiable at $x$. In particular, it follows from Remark 2.7 that if $S \leq 0$ and $X_{\lambda}$ is given by (2.16) with $u$ in place of $\varphi$, then $\left(D u(x)-\lambda n(x), X_{\lambda}+\mu n(x) \otimes n(x)\right) \in J_{\mathscr{O}}^{2,+} u(x)$ for $\lambda=\mu=0$ and for $\lambda>0, \mu \in \mathbb{R}$. Thus, assuming that $B$ is lower semicontinuous, $u$ will not be a subsolution unless

$$
B\left(x, u(x), D u(x)-\lambda n(x), X_{\lambda}+\mu n(x) \otimes n(x)\right) \leq 0 \text { for } \lambda \geq 0, \mu \in \mathbb{R}
$$

(to see that (7.3) holds with $\lambda=0$ and $\mu$ arbitrary, just let $\lambda \downarrow 0$ with $\mu$ arbitrary but fixed).

Notice, however, that if $B(x, r, p)$ is first order and $n \in \mathbb{R}^{N}$, then $B(x, u(x), D u(x)) \leq 0$ implies $B(x, u(x), D u(x)-\lambda n) \leq 0$ for $\lambda \geq 0$ provided

$$
\lambda \rightarrow B(x, r, p-\lambda n) \text { is nonincreasing in } \lambda \geq 0 .
$$

Moreover, by Remark 2.7, if $u$ is differentiable at $x$ and $(p, X) \in J_{\mathscr{\theta}}^{2,+} u(x)$, then $D u(x)-p$ is a "generalized normal" to $\mathscr{O}$ at $x$. Since analogous remarks evidently hold for supersolutions, we have found a case in which classical satisfaction of $(\mathrm{BC})$ implies $(\mathrm{BC})$ in the strong sense. More precisely, we have proved

Proposition 7.2. If $B \in C\left(\partial \Omega \times \mathbb{R} \times \mathbb{R}^{N}\right)$ satisfies (7.4) for every $x \in \partial \Omega, \quad r \in$ $\mathbb{R}, p \in \mathbb{R}^{N}$ and $n$ is a generalized normal to $\mathscr{O}$ at $x, u \in C^{1}(\bar{\Omega})$ and $B(x, u(x), D u(x)) \leq 0$ (respectively, $\geq 0$ ) for $x \in \partial \Omega$, then $u$ is a subsolution (respectively, supersolution) of (BC) in the strong sense.

This result will be needed in the construction of subsolutions and supersolutions below.

As mentioned above, it will be necessary to relax the interpretation of the boundary condition "in the strong sense;" one way to motivate this is to consider what happens when we try to pass to the limit in the following situation, which is an analogue of Remark 6.3 "with boundary:" Suppose $F_{n} \in$ $C\left(\bar{\Omega} \times \mathbb{R} \times \mathbb{R}^{N} \times \mathscr{S}(N)\right), B_{n} \in C\left(\partial \Omega \times \mathbb{R} \times \mathbb{R}^{N} \times \mathscr{S}(N)\right)$ for $n=1,2, \ldots$ and $u_{n}$ be a subsolution of $F_{n}=0$ in $\Omega, B_{n}=0$ on $\partial \Omega$ in the strong sense. Let $F_{n} \rightarrow F, B_{n} \rightarrow B$ uniformly on compact sets as $n \rightarrow \infty$ and $u=$ lim $\sup _{n \rightarrow \infty}^{*} u_{n}$ be bounded. What problem does $u$ solve? Of course, we know 
from Remark 6.3 that $u$ is a solution of $F \leq 0$ in $\Omega$. However, when we attempt to check the boundary condition $B(x, u(x), p, X) \leq 0$ for $x \in \partial \Omega$ and $(p, X) \in \bar{J}_{\bar{\Omega}}^{2,+} u(x)$, the proof fails. We know, from Proposition 4.3, that (passing to a subsequence if necessary), there exists $\left(p_{n}, X_{n}\right) \in \bar{J}_{\bar{\Omega}}^{2,+} u_{n}\left(x_{n}\right)$ such that $\left(x_{n}, u_{n}\left(x_{n}\right), p_{n}, X_{n}\right) \rightarrow(x, u(x), p, X)$, but we do not know $x_{n} \in \partial \Omega$ (and the example below shows this cannot be achieved in general). If $x_{n} \in \Omega$, we have $F_{n}\left(x_{n}, u_{n}\left(x_{n}\right), p_{n}, X_{n}\right) \leq 0$ (rather than $B_{n}\left(x_{n}, u_{n}\left(x_{n}\right), p_{n}, X_{n}\right) \leq 0$ ). However, in this way we can conclude that either

$$
B(x, u(x), p, X) \leq 0 \text { or } F(x, u(x), p, X) \leq 0 ;
$$

in other words, $u$ is a subsolution for the boundary condition

$$
F\left(x, u, D u, D^{2} u\right) \wedge B\left(x, u, D u, D^{2} u\right)=0
$$

in the strong sense (where $a \wedge b=\min \{a, b\}$ and $a \vee b=\max \{a, b\}$ ). The following example, in the context of a Neumann problem, shows that this is the best that can be expected.

Example 7.3. The linear problem

$$
-\varepsilon u^{\prime \prime}+u^{\prime}+u=x+1
$$

on $(0,1) \subset \mathbb{R}$ with the homogeneous Neumann conditions

$$
u^{\prime}(0)=u^{\prime}(1)=0
$$

has a unique classical solution $u_{\varepsilon}$ for $\varepsilon>0$ and it follows from Proposition 7.2 that $u_{\varepsilon}$ satisfies (7.6) in the strong sense when we chose the function $B$ that defines the boundary condition (7.6) to be given by $B(1, r, p)=p$ and $B(0, r, p)=-p$; note the monotonicity in the direction of the exterior normals to $(0,1)$. We will see that the limit $\lim _{\varepsilon \downarrow 0} u_{\varepsilon}=u$ exists uniformly on $[0,1]$ and the limit $u$ does not satisfy (7.6) in the strong sense.

It is elementary to compute

$$
u_{\varepsilon}(x)=x+\frac{e^{\lambda_{-}}-1}{\lambda_{+}\left(e^{\lambda_{+}}-e^{\lambda_{-}}\right)} e^{\lambda_{+} x}+\frac{1-e^{\lambda_{+}}}{\lambda_{-}\left(e^{\lambda_{+}}-e^{\lambda_{-}}\right)} e^{\lambda_{-} x},
$$

where $\lambda_{ \pm}=(1 / 2 \varepsilon)\left(1 \pm(1+4 \varepsilon)^{1 / 2}\right)$. Noting that $\lambda_{+} \rightarrow \infty$ and $\lambda_{-} \rightarrow-1$ as $\varepsilon \downarrow 0$, one sees that

$$
u_{\varepsilon}(x) \rightarrow u(x) \equiv x+e^{-x} \quad \text { uniformly on }[0,1] \text { as } \varepsilon \downarrow 0 \text {. }
$$

The function $u(x)=x+e^{-x}$ satisfies

$$
u^{\prime}+u=x+1 \text { in }(0,1) \text { and } u^{\prime}(0)=0
$$

in the classical sense. However, $u^{\prime}(1)=1-1 / e>0$ and therefore $u$ is not a subsolution of $B\left(1, u^{\prime}(1)\right)=0$ in the strong sense. Note, however, that $\left(u^{\prime}+u-(x+1)\right) \wedge u^{\prime} \leq 0$ does hold at $x=1$, in agreement with the above discussion.

These considerations suggest the appropriate definitions regarding solutions of (BVP). 
Definition 7.4. Let $\Omega$ be an open subset of $\mathbb{R}^{N}, F \in C\left(\bar{\Omega} \times \mathbb{R} \times \mathbb{R}^{N} \times \mathscr{S}(N)\right)$, and $B \in C\left(\partial \Omega \times \mathbb{R} \times \mathbb{R}^{N} \times \mathscr{S}(N)\right)$ be proper. Then $u$ is a (viscosity) subsolution of (BVP) if $u \in \operatorname{USC}(\bar{\Omega})$ and

$$
\left\{\begin{array}{l}
F(x, u(x), p, X) \leq 0 \text { for } x \in \Omega,(p, X) \in \bar{J}_{\bar{\Omega}}^{2,+} u(x), \\
F(x, u(x), p, X) \wedge B(x, u(x), p, X) \leq 0 \text { for } x \in \partial \Omega,(p, X) \in \bar{J}_{\bar{\Omega}}^{2,+} u(x) .
\end{array}\right.
$$

Similarly, $u$ is a supersolution of (BVP) if $u \in \operatorname{LSC}(\bar{\Omega})$ and

$\left\{\begin{array}{l}F(x, u(x), p, X) \geq 0 \text { for } x \in \Omega,(p, X) \in \bar{J}_{\bar{\Omega}}^{2,-} u(x), \\ F(x, u(x), p, X) \vee B(x, u(x), p, X) \geq 0 \text { for } x \in \partial \Omega,(p, X) \in \bar{J}_{\bar{\Omega}}^{2,-} u(x) .\end{array}\right.$

Finally, $u$ is a solution of (BVP) if it is both a subsolution and a supersolution.

Another way to express these definitions is to introduce the functions

$$
G_{-}(x, r, p, X)= \begin{cases}F(x, r, p, X) & \text { if } x \in \Omega, \\ F(x, r, p, X) \wedge B(x, r, p, X) & \text { if } x \in \partial \Omega\end{cases}
$$

and

$$
G_{+}(x, r, p, X)= \begin{cases}F(x, r, p, X) & \text { if } x \in \Omega, \\ F(x, r, p, X) \vee B(x, r, p, X) & \text { if } x \in \partial \Omega,\end{cases}
$$

and note that " $u$ is a subsolution of (BVP)" means exactly that $u$ is a solution of $G_{-} \leq 0$ in $\bar{\Omega}$, etc. Observe that $G_{-}$is lower semicontinuous and $G_{+}$ is upper semicontinuous. In fact, $G_{-}$is precisely the lower semicontinuous envelope of the function

$$
G(x, r, p, X)= \begin{cases}F(x, r, p, X) & \text { if } x \in \Omega \\ B(x, r, p, X) & \text { if } x \in \partial \Omega\end{cases}
$$

while $G_{+}$is the upper semicontinuous envelope of $G$.

7.B Existence and uniqueness for the Neumann problem. We are concerned here with the following choice $B=B(x, r, p)$ of the boundary condition $B$,

$$
B(x, r, p)=\langle n(x), p\rangle+f(x, r)
$$

where $f \in C(\partial \Omega \times \mathbb{R})$. With appropriate restrictions on $\Omega, F$, and $f$, we prove that (BVP) has a unique solution by a program analogous to that developed in $\S \S 3$ and 4 . Regarding $\Omega$ we will always assume that

$$
\bar{\Omega} \text { is a compact } C^{1} N \text {-submanifold with boundary of } \mathbb{R}^{N} \text {. }
$$

In addition, we impose the uniform exterior sphere condition

$$
\exists r>0 \text { such that } b(x+r n(x), r) \cap \Omega=\varnothing \text { for } x \in \partial \Omega
$$

where $b(x, r)$ denotes the closed ball of radius $r$ centered at $x$. For $F$ we will require (3.13), restated here for convenience,

$$
\begin{aligned}
& \gamma(r-s) \leq F(x, r, p, X)-F(x, s, p, X) \\
& \qquad \text { for } r \geq s,(x, p, X) \in \bar{\Omega} \times \mathbb{R}^{N} \times \mathscr{S}(N)
\end{aligned}
$$


as well as uniform continuity in $(p, X)$ near $\partial \Omega$; more precisely, we require that there be a neighborhood $V$ of $\partial \Omega$ relative to $\bar{\Omega}$ such that

$$
\left\{\begin{array}{l}
|F(x, r, p, X)-F(x, r, q, Y)| \leq \omega(|p-q|+\|X-Y\|) \\
\text { for } x \in V, p, q \in \mathbb{R}^{N}, X, Y \in \mathscr{S}(N)
\end{array}\right.
$$

for some $\omega:[0, \infty) \rightarrow[0, \infty]$ satisfying $\omega(0+)=0$. In addition, we strengthen (3.14) to the condition

$$
\left\{\begin{array}{l}
F(y, r, p, Y)-F(x, r, p, X) \leq \omega\left(\alpha|x-y|^{2}+|x-y|(|p|+1)\right) \\
\text { whenever } x, y \in \bar{\Omega}, r \in \mathbb{R}, p \in \mathbb{R}^{N}, X, Y \in \mathscr{S}(N), \text { and } \\
\text { (3.10) holds }
\end{array}\right.
$$

where we may use the same $\omega$ as in (7.15). Observe that (7.16) implies (3.14). For $f$ we assume that

$$
f(x, r) \text { is nondecreasing in } r \text { for } x \in \partial \Omega .
$$

We have:

Theorem 7.5. Let (7.11)-(7.17) hold. If $u$ is a subsolution of (BVP) and $v$ is a supersolution of (BVP), then $u \leq v$ in $\bar{\Omega}$. Moreover, (BVP) has a unique solution.

The proof consists of verifying the comparison assertion, then producing a subsolution and a supersolution, and invoking Perron's method. We begin with the comparison proof.

Proof of comparison. The strategy involves two types of approximations. As a first step, we produce approximations $u_{\varepsilon}, v_{\varepsilon}$ such that $u_{\varepsilon} \rightarrow u, v_{\varepsilon} \rightarrow v$ uniformly as $\varepsilon \downarrow 0$ and $u_{\varepsilon}, v_{\varepsilon}$ are a subsolution and a supersolution of (BVP) with $B(x, r, p)$ replaced by $B(x, r, p)+\varepsilon$ and $B(x, r, p)-\varepsilon$ respectively. (Please note that $B$ itself will retain its meaning below.) If we then can prove $u_{\varepsilon} \leq v_{\varepsilon}$ we find $u \leq v$ in the limit $\varepsilon \downarrow 0$. After this, we arrange to deal only with the inequalities involving $F$ (and not $B$ ) via another perturbation built into the "test functions" we will use.

Let us proceed with the first step. We need the following elementary lemma

Lemma 7.6. Let (7.12) hold and $\nu \in C\left(\partial \Omega, \mathbb{R}^{N}\right)$ satisfy $\langle n(x), \nu(x)\rangle>0$ on $\partial \Omega$. Then there is a function $\varphi \in C^{2}(\bar{\Omega})$ such that

$$
\langle\nu(x), D \varphi(x)\rangle \geq 1 \text { for } x \in \partial \Omega \text { and } \varphi \geq 0 \text { on } \bar{\Omega} \text {. }
$$

The proof of the lemma is deferred until the theorem is established. Choosing $\nu=n$ in Lemma 7.6, let $\varphi$ be provided by the lemma; we may assume that the support of $\varphi$ lies in $V$. Let $u$ be a solution of $G_{-} \leq 0$ in $\bar{\Omega}$ (that is, a subsolution of (BVP)) and $v$ a solution of $G_{+} \geq 0$, and put

$$
u_{\varepsilon}(x)=u(x)-\varepsilon \varphi(x)-C \text { and } v_{\varepsilon}(x)=v(x)+\varepsilon \varphi(x)+C
$$

where $C=C(\varepsilon)$ will be chosen later on. Using (7.14) and (7.15), for $x \in V$ and $(p, X) \in \bar{J}_{\bar{\Omega}}^{2,+} u_{\varepsilon}(x)$ we have

$$
F\left(x, u_{\varepsilon}(x), p, X\right) \leq F\left(x, u(x), p+\varepsilon D \varphi(x), X+\varepsilon D^{2} \varphi(x)\right)-\gamma C+\omega(\varepsilon M),
$$

where $M=\max _{\bar{\Omega}}\left(|D \varphi(x)|+\left\|D^{2} \varphi(x)\right\|\right)$ and, if $x \in \partial \Omega$, 


$$
\begin{aligned}
B\left(x, u_{\varepsilon}(x), p\right) & =B\left(x, u_{\varepsilon}(x), p+\varepsilon D \varphi(x)\right)-\varepsilon\langle D \varphi(x), n(x)\rangle \\
& \leq B(x, u(x), p+\varepsilon D \varphi(x))-\varepsilon .
\end{aligned}
$$

Using next that $\bar{J}_{\bar{\Omega}}^{2,+} u_{\varepsilon}(x)+\left(\varepsilon D \varphi(x), \varepsilon D^{2} \varphi(x)\right)=\bar{J}_{\bar{\Omega}}^{2,+} u(x)$ (see Remarks 2.7), we conclude that if $C=\omega(\varepsilon M) / \gamma$, then $u_{\varepsilon}$ is a subsolution of (BVP) with $B+\varepsilon$ in place of $B$. Similarly, $v_{\varepsilon}$ is a supersolution of (BVP) with $B$ replaced by $B-\varepsilon$.

We are reduced to proving that if $u$ is a subsolution and $v$ is a supersolution of (BVP) with $B$ replaced by $B+\varepsilon$ and $B-\varepsilon$ respectively, then $u \leq v$. Assume, to the contrary, that $\max _{\bar{\Omega}}(u-v)>0$. We know that $\max _{\partial \Omega}(u-v) \geq$ $\max _{\bar{\Omega}}(u-v)$ by $\S 5 . \mathrm{B}$, so there must exist $z \in \partial \Omega$ such that $u(z)-v(z)=$ $\max _{\bar{\Omega}}(u-v)>0$. Put

$\Phi(x, y)=u(x)-v(y)-\frac{\alpha}{2}|x-y|^{2}+f(z, u(z))\langle n(z), x-y\rangle-|x-z|^{4} \quad$ on $\bar{\Omega} \times \bar{\Omega}$, and let $\left(x_{\alpha}, y_{\alpha}\right)$ be a maximum point of $\Phi$. By Proposition 3.7, since $u(x)-$ $v(x)-|x-z|^{4}$ has $x=z$ as a unique maximum point,

$$
x_{\alpha} \rightarrow z, \quad \alpha\left|x_{\alpha}-y_{\alpha}\right|^{2} \rightarrow 0, \quad u\left(x_{\alpha}\right) \rightarrow u(z), \quad v\left(x_{\alpha}\right) \rightarrow v(z)
$$

as $\alpha \rightarrow \infty$. For simplicity, we now write $(\hat{x}, \hat{y})$ for $\left(x_{\alpha}, y_{\alpha}\right)$ and put

$$
\psi(x, y)=\frac{\alpha}{2}|x-y|^{2}-f(z, u(z))\langle n(z), x-y\rangle+|x-z|^{4} .
$$

We have not yet invoked the exterior sphere condition (7.13) and do so now. Restating (7.13) as

$$
|y-x-r n(x)|^{2}>r^{2} \text { for } x \in \partial \Omega \text { and } y \in \Omega,
$$

we see it is equivalent to

$$
\langle n(x), y-x\rangle<\frac{1}{2 r}|y-x|^{2} \quad \text { for } x \in \partial \Omega \text { and } y \in \Omega .
$$

Using (7.21) we compute that if $\hat{x} \in \partial \Omega$ then

$$
\begin{aligned}
B\left(\hat{x}, u(\hat{x}), D_{x} \psi(\hat{x}, \hat{y})\right)= & B(\hat{x}, u(\hat{x}), \alpha(\hat{x}-\hat{y}) \\
& \left.-f(z, u(z)) n(z)+4|\hat{x}-z|^{2}(\hat{x}-z)\right) \\
\geq & -\frac{\alpha}{2 r}|\hat{x}-\hat{y}|^{2}-f(z, u(z))\langle n(\hat{x}), n(z)\rangle \\
& +O\left(|\hat{x}-z|^{3}\right)+f(\hat{x}, u(\hat{x}))
\end{aligned}
$$

and in view of (7.20) this implies

$$
B\left(\hat{x}, u(\hat{x}), D_{x} \psi(\hat{x}, \hat{y})\right) \geq o(1) \quad \text { as } \alpha \rightarrow \infty \text { if } \hat{x} \in \partial \Omega .
$$

A similar computation (this time involving (7.17)) shows that

$$
B\left(\hat{y}, v(\hat{y}),-D_{y} \psi(\hat{x}, \hat{y})\right) \leq o(1) \quad \text { as } \alpha \rightarrow \infty \text { if } \hat{y} \in \partial \Omega .
$$

Recalling that $u$ is a subsolution with respect to $B+\varepsilon$, etc., and the definition of subsolutions and supersolutions, we conclude

$$
\begin{cases}F\left(\hat{x}, u(\hat{x}), D_{x} \psi(\hat{x}, \hat{y}), X\right) \leq 0 & \text { for }\left(D_{x} \psi(\hat{x}, \hat{y}), X\right) \in \overline{\bar{J}}_{\bar{\Omega}^{2+}} u(\hat{x}), \\ F\left(\hat{y}, v(\hat{y}),-D_{y} \psi(\hat{x}, \hat{y}), Y\right) \geq 0 & \text { for }\left(-D_{y} \psi(\hat{x}, \hat{y}), Y\right) \in \overline{\bar{J}}_{\bar{\Omega}}^{2,-} v(\hat{y}),\end{cases}
$$

provided $\alpha$ is large. 
The remaining arguments are very close to those given in the proof of Theorem 3.3. We apply Theorem 3.2 with $k=2, \mathscr{O}_{1}=\mathscr{O}_{2}=\bar{\Omega}, u_{1}=u, u_{2}=-v$, and $\varepsilon=1 / \alpha$ to find $X, Y \in \mathscr{S}(N)$ such that

$$
\left(D_{x} \psi(\hat{x}, \hat{y}), X\right) \in \bar{J}_{\bar{\Omega}}^{2,+}, \quad\left(-D_{y} \psi(\hat{x}, \hat{y}), Y\right) \in \bar{J}_{\bar{\Omega}}^{2,-} v(\hat{y})
$$

and

$$
-(\alpha+\|A\|)\left(\begin{array}{cc}
I & 0 \\
0 & I
\end{array}\right) \leq\left(\begin{array}{cc}
X & 0 \\
0 & -Y
\end{array}\right) \leq A+\frac{1}{\alpha} A^{2},
$$

where $A=D^{2} \psi(\hat{x}, \hat{y})$. Observing that

$$
A=\alpha\left(\begin{array}{cc}
I & -I \\
-I & I
\end{array}\right)+O\left(|\hat{x}-z|^{2}\right), \quad A^{2}=2 \alpha^{2}\left(\begin{array}{cc}
I & -I \\
-I & I
\end{array}\right)+O\left(\alpha|\hat{x}-z|^{2}+|\hat{x}-z|^{4}\right),
$$

we see from (7.23) that

$$
-3 \alpha\left(\begin{array}{cc}
I & 0 \\
0 & I
\end{array}\right)+o(1) \leq\left(\begin{array}{cc}
X & 0 \\
0 & -Y
\end{array}\right) \leq 3 \alpha\left(\begin{array}{cc}
I & -I \\
-I & I
\end{array}\right)+o(1) \quad \text { as } \alpha \rightarrow \infty .
$$

We want to use this information in conjunction with (7.22); to this end, we formulate the next lemma (which will be used later as well).

Lemma 7.7. Let (7.15) and (7.16) hold. Let $\delta>0, x, y \in V, r \in \mathbb{R}, p \in \mathbb{R}^{N}$, $X, Y \in \mathscr{S}(N)$, and

$$
-(3 \alpha+\delta)\left(\begin{array}{cc}
I & 0 \\
0 & I
\end{array}\right) \leq\left(\begin{array}{cc}
X & 0 \\
0 & -Y
\end{array}\right) \leq 3 \alpha\left(\begin{array}{cc}
I & -I \\
-I & I
\end{array}\right)+\delta\left(\begin{array}{cc}
I & 0 \\
0 & I
\end{array}\right)
$$

hold. Then

$F(y, r, p, Y)-F(x, r, p, X) \leq \omega\left(\left(\alpha+\frac{2}{3} \delta\right)|x-y|^{2}+|x-y|(|p|+1)\right)+2 \omega(\delta)$.

Proof. By assumption,

$$
-(3 \alpha+2 \delta)\left(\begin{array}{ll}
I & 0 \\
0 & I
\end{array}\right) \leq\left(\begin{array}{cc}
X-\delta I & 0 \\
0 & -Y-\delta I
\end{array}\right) \leq 3 \alpha\left(\begin{array}{cc}
I & -I \\
-I & I
\end{array}\right) .
$$

Using (7.16) we therefore have

$F(y, r, p, Y+\delta I)-F(x, r, p, X-\delta I) \leq \omega\left(\left(\alpha+\frac{2}{3} \delta\right)|x-y|^{2}+|x-y|(|p|+1)\right)$.

Next, (7.15) implies

$F(y, r, p, Y+\delta I)-F(x, r, p, X-\delta I) \geq F(y, r, p, Y)-F(x, r, p, X)-2 \omega(\delta)$

and the result follows.

We complete the proof of comparison. From the lemma and the developments above,

$$
\begin{aligned}
0 & \leq F\left(\hat{y}, v(\hat{y}),-D_{y} \psi(\hat{x}, \hat{y}), Y\right)-F\left(\hat{x}, u(\hat{x}), D_{x} \psi(\hat{x}, \hat{y}), X\right) \\
& \leq F\left(\hat{y}, u(\hat{x}),-D_{y} \psi(\hat{x}, \hat{y}), Y\right)-F\left(\hat{x}, u(\hat{x}),-D_{y} \psi(\hat{x}, \hat{y}), X\right) \\
& -\gamma(u(\hat{x})-v(\hat{y}))+\omega\left(4|\hat{x}-z|^{3}\right) \\
& \leq \omega\left(\alpha|\hat{x}-\hat{y}|^{2}+|\hat{x}-\hat{y}|\left(\left|D_{y} \psi(\hat{x}, \hat{y})\right|+1\right)\right)-\gamma(u(\hat{x})-v(\hat{y}))+o(1)
\end{aligned}
$$

as $\alpha \rightarrow \infty$. From this we obtain a contradiction as in the proof of Theorem 3.3. 
Proof of existence. In order to establish existence, we may use Perron's Method from Theorem 4.1 as supplemented by Remark 4.5 to deduce that it suffices to produce a subsolution and a supersolution. Using Proposition 7.2, we see it suffices to produce a classical subsolution and supersolution. Let $\varphi \in C^{2}(\bar{\Omega})$ be as in Lemma 7.6. Put

$$
\underline{u}(x)=-A \varphi(x)-B, \quad \bar{u}(x)=A \varphi(x)+B
$$

where $A, B$ are constants to be determined. Observing that

$$
B(x, \bar{u}(x), D \bar{u}(x)) \geq A\langle n(x), D \varphi(x)\rangle+f(x, 0) \geq A+f(x, 0)
$$

for $x \in \partial \Omega$ and

$$
F\left(x, \bar{u}(x), D \bar{u}(x), D^{2} \bar{u}(x)\right) \geq F\left(x, 0, A D \varphi(x), A D^{2} \varphi(x)\right)+\gamma B
$$

for $x \in \bar{\Omega}$ we see that if we first choose $A$ and then $B$ by

$$
A=\sup _{\partial \Omega}|f(x, 0)|, \quad B=\frac{1}{\gamma} \sup _{\bar{\Omega},|p|+\|X\| \leq A M} \mid F(x, 0, p, X \mid,
$$

where $M=\sup _{\bar{\Omega}}\left(\mid D \varphi(x)+\left\|D^{2} \varphi(x)\right\|\right)$, then $\bar{u}$ is a supersolution. Likewise, $\underline{u}$ will be a subsolution. It remains to prove Lemma 7.6.

Proof of Lemma 7.6. In view of (7.12), there is a $C^{1}$ function $\psi$ on $\mathbb{R}^{N}$ such that $\psi \geq 0$ on $\mathbb{R}^{N} \backslash \Omega, \psi<0$ on $\Omega$, and $|D \psi|>0$ on $\partial \Omega$. Multiplying by a constant if necessary, we may also assume that $|D \psi(x)|>\langle\nu(x), n(x)\rangle^{-1}$ for $x \in \partial \Omega$. By standard approximation theorems, we see that there is a sequence of $C^{2}$ functions $\psi_{k}$ such that $\psi_{k} \rightarrow \psi$ in $C^{1}(\bar{\Omega})$ as $k \rightarrow \infty$. In particular, we have

$$
\left\langle\nu(x), D \psi_{k}(x)\right\rangle \rightarrow\langle\nu(x), D \psi(x)\rangle \quad \text { uniformly on } \partial \Omega \text { as } k \rightarrow \infty \text {. }
$$

Since $n(x)=D \psi(x) /|D \psi(x)|$ and $|D \psi(x)|\langle\nu(x), n(x)\rangle>1$ for $x \in \partial \Omega$, if $k$ and then $C$ are sufficiently large, $\varphi=\psi_{k}+C$ has the desired properties.

7.C. The generalized Dirichlet problem. We turn to the Dirichlet problem

$$
\begin{cases}F\left(x, u, D u, D^{2} u\right)=0 & \text { in } \Omega \\ u-f(x)=0 & \text { on } \partial \Omega\end{cases}
$$

where $f \in C(\partial \Omega)$.

The simplest example of this problem with a unique solution that does not satisfy boundary conditions in the strong sense is the following: $F\left(x, u, D u, D^{2} u\right)$ $=u-h(x)$ and $f(x) \equiv 0$. If $h$ is continuous on $\bar{\Omega}$, then $u \equiv h$ is evidently the only solution of this problem and it does not satisfy $u=0$ on $\partial \Omega$ in the strong sense unless $h=0$ on $\partial \Omega$.

We will provide a comparison theorem, Theorem 7.9 below, with this example as a special case. This comparison theorem, however will be different in character from those we have obtained so far in that it will assert comparison for a continuous subsolution and supersolution. As we know, the ability to compare semicontinuous subsolutions and supersolutions allows us to prove existence. Thus the following counterexample to existence indicates that the restriction to continuous functions (or some other assumption) is necessary in Theorem 7.9. 
Example 7.8. Let $N=2$ and $\Omega=\{(x, y):-1<x<1,0<y<1\}$. Let $f \in C(\partial \Omega)$ satisfy $0 \leq f \leq 1, f(x, 0)=0$, and $f(x, 1)=1$ for $|x|<1$. We claim that the problem

$$
\begin{cases}u+x u_{y}=0 & \text { in } \Omega, \\ u=f & \text { on } \partial \Omega\end{cases}
$$

does not have a solution. To see this, suppose the contrary and let $u(x, y)$ be a solution. We claim that then for fixed $|x|<1, v(y)=u(x, y)$ is a solution of

$$
v+x v^{\prime}=0 \text { in }(0,1), \quad v(0)=0, \quad v(1)=1 .
$$

Assume for the moment that this is the case. Then we remark that Theorem 7.9 below shows that $v$ is uniquely determined by (7.26), $v \equiv 0$ is a solution of (7.26) if $x>0$ and $v(y)=e^{(1-y) / x}$ is a solution of (7.26) if $x<0$ (these last assertions the reader can check by computations). Thus we see that $u(x, y)=$ 0 if $x>0$ and $u(x, y)=e^{(1-y) / x}$ if $x<0$; however, $u$ cannot then be continuous at $(0,1)$ and we conclude that $(7.25)$ does not have a solution.

It remains to verify that if $u$ is a solution of (7.25), then $v$ is a solution of (7.26). One way to check this is as follows: Fix $\bar{x} \in(-1,1)$ and set $v(y)=$ $u(\bar{x}, y)$. Let $\varphi \in C^{2}([0,1])$ and assume that $v-\varphi$ has a unique maximum at some point $\bar{y} \in[0,1]$. For $\alpha>0$ let $(\hat{x}, \hat{y})$ be a maximum point of the function $u(x, y)-\varphi(y)-\alpha|x-\bar{x}|^{2}$. It follows from Proposition 3.7 that then $\hat{x} \rightarrow \bar{x}, \hat{y} \rightarrow \bar{y}$ as $\alpha \rightarrow \infty$; moreover, we have $u(\hat{x}, \hat{y})+\hat{x} \varphi^{\prime}(\hat{y}) \leq 0$. From this information we deduce that $v(\bar{y})+\bar{x} \varphi^{\prime}(\bar{y}) \leq 0$ and conclude that $v$ is a subsolution of (7.26) with $x=\bar{x}$. In a similar way, one shows that $v$ is a supersolution. Finally we remark that $\bar{u} \equiv 1, \underline{u} \equiv 0$ are, respectively, a supersolution and a subsolution of (7.25). Thus comparison of semicontinuous semisolutions of (7.25) must fail. (If the reader is concerned about regularity questions and the "corners," note that the corners can be smoothed up without changing the essential points of the discussion.)

We turn to the comparison result for continuous solutions.

Theorem 7.9. Let (7.12), (7.14), (7.15), and (7.16) hold. If $u, v \in C(\bar{\Omega}), u$ is a subsolution of (7.24) and $v$ is a supersolution of (7.24), then $u \leq v$.

Proof. As before, we argue by contradiction and so suppose that $\max _{\bar{\Omega}}(u-v)>$ 0 . We may assume that $\max _{\bar{\Omega}}(u-v)=u(z)-v(z)>0$ for some $z \in \partial \Omega$. We divide our considerations into two cases.

First, we consider the case when $v(z)<f(z)$. For $\alpha>1$ and $0<\varepsilon<1$, we define the function

$$
\Phi(x, y)=u(x)-v(y)-|\alpha(x-y)+\varepsilon n(z)|^{2}-\varepsilon|y-z|^{2} \quad \text { on } \bar{\Omega} \times \bar{\Omega} .
$$

Let $(\hat{x}, \hat{y})$ be a maximum point of $\Phi$. We assume $\alpha$ is so large that $z-$ $(\varepsilon / \alpha) n(z) \in \Omega$. The inequality $\Phi(\hat{x}, \hat{y}) \geq \Phi(z-(\varepsilon / \alpha) n(z), z)$ reads

$$
|\alpha(\hat{x}-\hat{y})+\varepsilon n(z)|^{2}+\varepsilon|\hat{y}-z|^{2} \leq u(\hat{x})-v(\hat{y})-u\left(z-\frac{\varepsilon}{\alpha} n(z)\right)+v(z)
$$

from which it follows (using the continuity of $u$ !) that if $\varepsilon$ is fixed, $\hat{x}, \hat{y} \rightarrow z$ and $\alpha(\hat{x}-\hat{y})+\varepsilon n(z) \rightarrow 0$ as $\alpha \rightarrow \infty$. Indeed, it is clear that $\alpha(\hat{x}-\hat{y})$ remains 
bounded as $\alpha \rightarrow \infty$, so assuming that $\alpha(\hat{x}-\hat{y}) \rightarrow w, \hat{x}, \hat{y} \rightarrow \tilde{z}$ (along a subsequence) as $\alpha \rightarrow \infty$, one finds

$$
|w+\varepsilon n(z)|^{2}+\varepsilon|\tilde{z}-z|^{2} \leq u(\tilde{z})-v(\tilde{z})-u(z)+v(z) \leq 0,
$$

where the last inequality follows from the definition of $z$, whence the claim is immediate. From this we see that $\hat{x}=\hat{y}-(\varepsilon n(z)+o(1)) / \alpha$ as $\alpha \rightarrow \infty$ and hence that $\hat{x} \in \Omega$ if $\alpha$ is large enough. Since $v(z)<f(z)$, we have (using the continuity of $v$ ) $v(\hat{y})<f(\hat{y})$ if $\alpha$ is large enough. Thus, if $\alpha$ is large enough, we have

$$
F(\hat{x}, u(\hat{x}), p, X) \leq 0 \text { for }(p, X) \in \bar{J}_{\bar{\Omega}}^{2,+} u(\hat{x})
$$

and

$$
F(\hat{y}, v(\hat{y}), q, Y) \geq 0 \text { for }(q, Y) \in \bar{J}_{\bar{\Omega}}^{2,-} v(\hat{y}) \text {. }
$$

Observe that if we set $\varphi(x, y)=|\alpha(x-y)+\varepsilon n(z)|^{2}+\varepsilon|y-z|^{2}$, then

$$
\begin{gathered}
D_{x} \varphi(x, y)=2 \alpha(\alpha(x-y)+\varepsilon n(z)), \\
-D_{y} \varphi(x, y)=2 \alpha(\alpha(x-y)+\varepsilon n(z))-2 \varepsilon(y-z),
\end{gathered}
$$

and

$$
D^{2} \varphi(x, y)=2 \alpha^{2}\left(\begin{array}{cc}
I & -I \\
-I & I
\end{array}\right)+2 \varepsilon\left(\begin{array}{ll}
0 & 0 \\
0 & I
\end{array}\right) .
$$

Now, using Theorem 3.2 together with Lemma 7.7, calculating as usual, sending $\alpha \rightarrow \infty$ and then $\varepsilon \downarrow 0$, we obtain a contradiction.

It remains to treat the case when $v(z) \geq f(z)$. Since $u(z)>v(z)$ this entails $u(z)>f(z)$. Replacing the above $\Phi$ by

$$
\Phi(x, y)=u(x)-v(y)-|\alpha(x-y)-\varepsilon n(z)|^{2}-\varepsilon|x-z|^{2},
$$

we argue as above and obtain a contradiction.

7.C'. The state constraints problem. The problem

$$
\begin{cases}F\left(x, u, D u, D^{2} u\right) \leq 0 & \text { in } \Omega, \\ F\left(x, u, D u, D^{2} u\right) \geq 0 & \text { in } \bar{\Omega}\end{cases}
$$

corresponds to an important problem in optimal control, the so-called state constraints problem provided $F$ has the form of the left member of (1.10). This is, indeed, the extreme form of (7.24) where $f(x) \equiv-\infty$. To make this clear, we give the definitions precisely. A function $u \in \operatorname{USC}(\bar{\Omega})$ (respectively, $v \in \operatorname{LSC}(\bar{\Omega})$ ) is called a subsolution (supersolution) of (7.27) if

$$
F(x, u(x), p, X) \leq 0 \text { for } x \in \bar{\Omega} \text { and }(p, X) \in \bar{J}^{2,+} u(x)
$$

(respectively,

$$
\left.F(x, v(x), p, X) \geq 0 \text { for } x \in \Omega \text { and }(p, X) \in \bar{J}_{\bar{\Omega}}^{2,-} v(x)\right) .
$$

An application of Theorem 7.9 immediately yields

Theorem 7.10. Let (7.12), (7.14), (7.15), and (7.16) hold. If $u, v \in C(\overline{\mathbf{\Omega}}), u$ is a subsolution of (7.27) and $v$ is a supersolution of (7.27), then $u \leq v$ on $\bar{\Omega}$. 
Indeed, $v$ is a supersolution and $u$ is a subsolution of (7.24) with $f(x) \geq$ $u(x)$ on $\partial \Omega$.

In fact, a subsolution $v$ of (7.27) is a supersolution of (7.24) for any $f \in$ $C(\partial \Omega)$ and hence a "universal bound" on all solutions. This implies that solutions of (7.27) do not exist in general. For example, if $F$ is a linear uniformly elliptic operator, (7.24) is uniquely solvable for arbitrary $f \in C(\partial \Omega)$ and then there can be no universal bound that is continuous in $\bar{\Omega}$. On the other hand, if $F$ is suitably degenerate in the normal direction at $\partial \Omega$, one can sometimes find such a supersolution.

7.D. A remark (BC) in the classical sense. The reader will have noticed by now that we gave some examples of solutions of $(B C)$ that were smooth but did not satisfy the boundary condition in the classical sense. In these examples, the equation $(E)$ is rather degenerate. In fact, "degeneracy" of a sort is necessary for this to happen, as we now establish.

Proposition 7.11. Let $B \in C\left(\partial \Omega \times \mathbb{R} \times \mathbb{R}^{N}\right), F$ be proper, and $u \in C^{2}(\bar{\Omega})$ be a subsolution (respectively, supersolution) of (BVP). If

$$
\left\{\begin{array}{l}
\lim \sup _{\mu \rightarrow \infty} F(x, r, p, X-\mu n \otimes n)>0 \\
\text { for }(x, r, p, X) \in \partial \Omega \times \mathbb{R} \times \mathbb{R}^{N} \times \mathscr{S}(N), n \in \mathbb{R}^{N} \backslash\{0\}
\end{array}\right.
$$

(respectively,

$$
\left\{\begin{array}{l}
\lim \inf _{\mu \rightarrow \infty} F(x, r, p, X+\mu n \otimes n)<0 \\
\text { for } \left.(x, r, p, X) \in \partial \Omega \times \mathbb{R} \times \mathbb{R}^{N} \times \mathscr{S}(N), n \in \mathbb{R}^{N} \backslash\{0\}\right),
\end{array}\right.
$$

then $u$ is a classical subsolution (respectively, supersolution) of (BC).

Proof. It will suffice to treat the subsolution case. We rely again on Remarks 2.7(iii). Let $y \in \mathbb{R}^{N} \backslash \bar{\Omega}$ and $z \in \partial \Omega$ be a nearest point to $y$ in $\bar{\Omega}$. It is clear that the set of such nearest points (as $y$ varies) is dense in $\partial \Omega$. Then $\bar{\Omega} \subset\left\{x \in \mathbb{R}^{N}:|x-y| \geq|y-z|\right\}=\mathscr{O}$ where the last equality is the definition of $\mathscr{O}$; now this inclusion evidently implies $J_{\mathscr{O}}^{2,+}$ Zero $\subset \bar{J}_{\bar{\Omega}}^{2,+} \operatorname{Zero}(z)$. On the other hand, we computed $J_{\mathscr{O}}^{2,+} \operatorname{Zero}(z)$ in Remark 2.7-with the notations $n=(y-z) /|y-z|$ and $r=|y-z|$, this computation shows that

$$
\left(-\lambda n, \frac{\lambda}{r} I+\mu n \otimes n\right) \in J_{\mathscr{O}}^{2,+} \operatorname{Zero}(z)
$$

for $\lambda>0$ and $\mu \in \mathbb{R}$. Thus the assumption that $u$ is a supersolution implies that

$B(z, u(z), D u(z)-\lambda n) \wedge F\left(z, u(z), D u(z)-\lambda n, D^{2} u(z)+\frac{\lambda}{r} I+\mu n \otimes n\right) \leq 0$

for $\lambda>0$ and $\mu \in \mathbb{R}$. Taking the limit superior as $\mu \rightarrow-\infty$ and using the fact that assumption (7.28) implies $B(z, u(z), D u(z)-\lambda n) \leq 0$ for $\lambda>0$ and then letting $\lambda \downarrow 0$ we find $B(z, u(z), D u(z)) \leq 0$. Since $z$ was an arbitrary "nearest point" and these are dense in $\partial \Omega$, the proof is complete. The reader will notice that it suffices to choose $n$ in (7.28) and (7.29) from $N_{\bar{\Omega}}(x)$.

7.E. Fully nonlinear boundary conditions. We conclude this section by describing an extremely general existence and uniqueness result for a fully nonlinear first-order boundary operator of the form $B=B(x, p), B \in C\left(\partial \Omega \times \mathbb{R}^{N}\right)$. 
We will require that $B$ satisfies

$B$ is uniformly continuous in $p$ uniformly in $x \in \partial \Omega$

and

$$
|B(x, p)-B(y, p)| \leq \omega(|x-y|(1+|p|))
$$

for some $\omega:[0, \infty) \rightarrow[0, \infty)$ satisfying $\omega(0+)=0$ and for some $\nu>0$

$$
B(x, p+\lambda n(x)) \geq B(x, p)+\nu \lambda \text { for } x \in \partial \Omega, \lambda \geq 0, p \in \mathbb{R}^{N} .
$$

We will also need to strengthen a bit the regularity of $\Omega$ by assuming that

$$
\partial \Omega \text { is of class } C^{1,1} \text {. }
$$

Observe that (7.33) is stronger than (7.12) and (7.13) since $\Omega$ is bounded. The loss of generality, however, is not really more restrictive for applications, since $\Omega$ typically is either smooth or has "corners," and corners are not allowed by (7.12) in any case. (Corners typically require some ad hoc analysis.)

We have

Theorem 7.12. Let (7.30)-(7.33) and (7.14)-(7.16) hold. If $u$ is a subsolution of (BVP) and $v$ is a supersolution of (BVP), then $u \leq v$ in $\bar{\Omega}$. Moreover, (BVP) has a unique solution.

Example 7.13. The Neumann boundary conditions of (7.11) are special cases of those considered above; note that (7.31) holds since $n$ is Lipschitz continuous on $\partial \Omega$ because of (7.33). Similarly, this setting includes the more general linear oblique derivative boundary condition corresponding to

$$
B(x, p)=\langle\gamma(x), p\rangle+f(x)
$$

where $\gamma$ is Lipschitz and satisfies

$$
\langle\gamma(x), n(x)\rangle \geq \nu>0 \text { for } x \in \partial \Omega ;
$$

note that $f$ is merely required to be continuous on $\partial \Omega$.

Nonlinear examples arising in optimal control and differential games are typically written in the form

$$
B(x, p)=\sup _{\alpha}\left(\left\langle\gamma_{\alpha}(x), p\right\rangle+f_{\alpha}(x)\right)
$$

and

$$
B(x, p)=\inf _{\beta} \sup _{\alpha}\left(\left\langle\gamma_{\alpha, \beta}(x), p\right\rangle+f_{\alpha, \beta}(x)\right)
$$

where $\gamma_{\alpha}, f_{\alpha}, \gamma_{\alpha, \beta}, f_{\alpha, \beta}$ are functions that satisfy the conditions (including continuity) laid on $\gamma, f$ above uniformly in the parameters $\alpha, \beta$.

As a last example, we offer the famous capillarity boundary condition that corresponds to

$$
B(x, p)=\langle n(x), p\rangle-\kappa(x)\left(1+|p|^{2}\right)^{1 / 2}
$$

where $\kappa \in C(\partial \Omega)$ and $\sup _{\partial \Omega}|\kappa|<1$.

We will not prove Theorem 7.12 since its proof, while based on the strategies presented above, requires some rather complex adaptations of them. We remark that the form of the boundary operator $B(x, p)$ could be generalized to $B(x, r, p)$ above, provided that $B$ is nondecreasing with respect to $r \in \mathbb{R}$ and (7.30)-(7.32) hold uniformly in bounded $r$. 
Notes on $\S 7$. Boundary conditions in the viscosity sense first appeared in P. L. Lions [121] and B. Perthame and R. Sanders [142] for Neumann or oblique derivative problems for first order equations, making this problem well posed! A general formulation was given later by $\mathbf{H}$. Ishii [90]. Existence and uniqueness was shown in P. L. Lions [121] (for the first-order case), H. Ishii and P. L. Lions [96] (second-order case), and P. Dupuis and H. Ishii [60, 61] (second-order and nonsmooth domains). However, it is worth emphasizing that the presentation we give using Theorem 3.2 simplifies the arguments substantially (even in this special case).

Concerning the Dirichlet problem, most of the works required (and often still require) continuous solutions up to the boundary and prescribed data on the entire boundary. It was first noted in P. L. Lions [116], G. Barles [14, 15] and $H$. Ishii [86] that this can be achieved for special classes of equations by imposing compatibility conditions on the boundary data or, in general, by assuming the existence of appropriate super and subsolutions. It was also noted in M. G. Crandall and R. Newcomb [58] and P. E. Souganidis [156] that some part of the boundary may be irrelevant (in the case of a first-order equation).

Progress on the understanding of Dirichlet boundary conditions was stimulated by the "state constraints" problems studied first by M. Soner [149, 150] and later by I. Capuzzo-Dolcetta and P. L. Lions [44]. This led to the "true" viscosity formulation of Dirichlet conditions as considered in $\mathbf{H}$. Ishii [90] and G. Barles and B. Perthame [25, 26]. This formulation, in some sense, automatically selects the relevant part of the boundary for degenerate problems and yields uniqueness for continuous solutions. However, the existence of a continuous solution fails in general (Example 7.8), and the situation is not entirely clear except for first-order optimal control problems [25, 26, 90].

We also mention at this stage that some uniqueness results for semicontinuous solutions have begun to emerge (G. Barles and B. Perthame $[25,26]$ and E. N. Barron and R. Jensen [33, 34]) for optimal control problems and the study of some second-order nondegenerate state constraints problems (J. M. Lasry and P. L. Lions [1 111$])$.

Finally, the possibility of solving general equations with general fully nonlinear oblique derivative type boundary conditions-a rather startling fact-was illustrated in G. Barles and P. L. Lions [24] for first-order equations. The full second-order result of Theorem 7.12 is a generalization by G. Barles [21] of a similar result of $\mathrm{H}$. Ishii [91].

\section{PARABOlic PROBLemS}

In this section we indicate how to extend the results of the preceeding sections to problems involving the parabolic equation

$$
u_{t}+F\left(t, x, u, D u, D^{2} u\right)=0
$$

where now $u$ is to be a function of $(t, x)$ and $D u, D^{2} u$ mean $D_{x} u(t, x)$ and $D_{x}^{2} u(t, x)$. We do this by discussing comparison for the Cauchy-Dirichlet problem on a bounded domain; it will then be clear how to modify other proofs as well. Let $\mathscr{O}$ be a locally compact subset of $\mathbb{R}^{N}, T>0$, and $\mathscr{O}_{T}=(0, T) \times \mathscr{O}$. We denote by $\mathscr{P}_{\mathscr{O}}^{2,+}, \mathscr{P}_{\mathscr{O}}^{2,-}$ the "parabolic" variants of the semijets $J_{\mathscr{O}}^{2,+}, J_{\mathscr{O}}^{2,-}$; for example, if $u: \mathscr{O}_{T} \rightarrow \mathbb{R}$ then $\mathscr{P}_{\mathscr{O}}^{2,+} u$ is defined 
by $(a, p, X) \in \mathbb{R} \times \mathbb{R}^{N} \times \mathscr{S}(N)$ lies in $\mathscr{P}_{\mathscr{O}}^{2,+} u(s, z)$ if $(s, z) \in \mathscr{O}_{T}$ and

$$
\begin{gathered}
u(t, x) \leq u(s, z)+a(t-s)+\langle p, x-z\rangle+\frac{1}{2}\langle X(x-z), x-z\rangle \\
+o\left(|t-s|+|x-z|^{2}\right) \quad \text { as } \mathscr{O}_{T} \ni(t, x) \rightarrow(s, z) ;
\end{gathered}
$$

similarly, $\mathscr{P}_{\mathscr{\theta}}^{2,-} u=-\mathscr{P}_{\mathscr{\theta}}^{2,+}(-u)$. The corresponding definitions of $\overline{\mathscr{P}}_{\mathscr{O}}^{2,+}$, $\overline{\mathscr{P}}_{\mathscr{O}}^{2,-}$ are then clear.

A subsolution of $(\mathrm{PE})$ on $\mathscr{O}_{T}$ is a function $u \in \mathrm{USC}\left(\mathscr{O}_{T}\right)$ such that

$a+F(t, x, u(t, x), p, X) \leq 0$ for $(t, x) \in \mathscr{O}_{T}$ and $(a, p, X) \in \mathscr{P}_{\mathscr{O}}^{2,+} u(t, x)$;

likewise, a supersolution is a function $v \in \operatorname{LSC}\left(\mathscr{O}_{T}\right)$ such that

$a+F(t, x, v(t, x), p, X) \geq 0$ for $(t, x) \in \mathscr{O}_{T}$ and $(a, p, X) \in \mathscr{P}_{\mathscr{O}}^{2,-} v(t, x)$;

and a solution is a function that is simultaneously a subsolution and a supersolution.

Remark 8.1. Suppose we set $x_{0}=t$ and $\tilde{x}=\left(x_{0}, x\right)$. Then (PE) is an equation of the form $\hat{F}\left(\tilde{x}, u, D_{\tilde{x}} u, D_{\tilde{x}}^{2} u\right)=u_{x_{0}}+\cdots$ that is proper if and only if $F\left(t, x, u, D u, D^{2} u\right)$ is proper when $t$ is held fixed. The definition of subsolutions, etc., of (PE), which takes into account that only the first derivative with respect to $t$ appears, does not coincide with the definition of subsolutions, etc., of $\hat{F}=0$ under this correspondence. However, it is not hard to see that the two notions are equivalent.

We will illustrate the additional considerations that arise in dealing with the Cauchy-Dirichlet problem for (PE) as opposed to the pure Dirichlet problem in the elliptic case. The problem of interest has the form

$$
\left\{\begin{array}{l}
(\mathrm{E}) \quad u_{t}+F\left(t, x, u, D u, D^{2} u\right)=0 \text { in }(0, T) \times \Omega, \\
(\mathrm{BC}) \quad u(t, x)=0 \text { for } 0 \leq t<T \text { and } x \in \partial \Omega, \\
(\mathrm{IC}) \quad u(0, x)=\psi(x) \text { for } x \in \bar{\Omega},
\end{array}\right.
$$

where $\Omega \subset \mathbb{R}^{N}$ is open and $T>0$ and $\psi \in C(\bar{\Omega})$ are given. By a subsolution of $(8.4)$ on $[0, T) \times \bar{\Omega}$ we mean a function $u \in \operatorname{USC}([0, T) \times \bar{\Omega})$ such that $u$ is a subsolution of $(\mathrm{E}), u(t, x) \leq 0$ for $0 \leq t<T$ and $x \in \partial \Omega$ and $u(0, x) \leq \psi(x)$ for $x \in \bar{\Omega}$-the appropriate notions of supersolutions and solutions are then obvious.

Theorem 8.2. Let $\Omega \subset \mathbb{R}^{N}$ be open and bounded. Let $F \in C([0, T] \times \bar{\Omega} \times \mathbb{R} \times$ $\mathbb{R}^{N} \times \mathscr{S}(N)$ ) be continuous, proper, and satisfy (3.14) for each fixed $t \in[0, T)$, with the same function $\omega$. If $u$ is a subsolution of (8.4) and $v$ is a supersolution of (8.4), then $u \leq v$ on $[0, T) \times \Omega$.

To continue, we require the parabolic analogue of Theorem 3.2. It takes the following form

Theorem 8.3. Let $u_{i} \in \operatorname{USC}\left((0, T) \times \mathscr{O}_{i}\right)$ for $i=1, \ldots, k$ where $\mathscr{O}_{i}$ is a locally compact subset of $\mathbb{R}^{N_{i}}$. Let $\varphi$ be defined on an open neighborhood of $(0, T) \times \mathscr{O}_{1} \times \cdots \times \mathscr{O}_{k}$ and such that $\left(t, x_{1}, \cdots, x_{k}\right) \rightarrow \varphi\left(t, x_{1}, \cdots, x_{k}\right)$ is once continuously differentiable in $t$ and twice continuously differentiable in 
$\left(x_{1}, \cdots, x_{k}\right) \in \mathscr{O}_{1} \times \cdots \times \mathscr{O}_{k}$. Suppose that $\hat{t} \in(0, T), \hat{x}_{i} \in \mathscr{O}_{i}$ for $i=1, \ldots, k$ and

$$
\begin{aligned}
w\left(t, x_{1}, \ldots, x_{k}\right) & \equiv u_{1}\left(t, x_{1}\right)+\cdots+u_{k}\left(t, x_{k}\right)-\varphi\left(t, x_{1}, \ldots, x_{k}\right) \\
& \leq w\left(\hat{t}, \hat{x}_{1}, \ldots, \hat{x}_{k}\right)
\end{aligned}
$$

for $0<t<T$ and $x_{i} \in \mathscr{O}$. Assume, moreover, that there is an $r>0$ such that for every $M>0$ there is a $C$ such that for $i=1, \ldots, k$

$$
\begin{aligned}
& b_{i} \leq C \text { whenever }\left(b_{i}, q_{i}, X_{i}\right) \in \mathscr{P}_{\mathscr{\theta}}^{2,+} u_{i}\left(t, x_{i}\right), \\
& \left|x_{i}-\hat{x}_{i}\right|+|t-\hat{t}| \leq r \text { and }\left|u_{i}\left(t, x_{i}\right)\right|+\left|q_{i}\right|+\left\|X_{i}\right\| \leq M .
\end{aligned}
$$

Then for each $\varepsilon>0$ there are $X_{i} \in \mathscr{S}\left(N_{i}\right)$ such that

$$
\left\{\begin{array}{c}
\text { (i) } \quad\left(b_{i}, D_{x_{i}} \varphi\left(\hat{t}, \hat{x}_{1}, \ldots, \hat{x}_{k}\right), X_{i}\right) \in \overline{\mathscr{P}}_{\hat{O}_{i}}^{2,+} u_{i}\left(\hat{t}, \hat{x}_{i}\right) \text { for } i=1, \ldots, k, \\
\text { (ii) }-\left(\frac{1}{\varepsilon}+\|A\|\right) I \leq\left(\begin{array}{ccc}
X_{1} & \ldots & 0 \\
\vdots & \ddots & \vdots \\
0 & \ldots & X_{k}
\end{array}\right) \leq A+\varepsilon A^{2}, \\
\text { (iii) } b_{1}+\cdots+b_{k}=\varphi_{t}\left(\hat{t}, \hat{x}_{1}, \ldots, \hat{x}_{k}\right),
\end{array}\right.
$$

where $A=\left(D_{x}^{2} \varphi\right)\left(\hat{t}, \hat{x}_{1}, \ldots, \hat{x}_{k}\right)$.

Observe that the condition (8.5) is guaranteed by having each $u_{i}$ be a subsolution of a parabolic equation.

Proof of Theorem 8.2. We first observe that for $\varepsilon>0, \tilde{u}=u-\varepsilon /(T-t)$ is also a subsolution of (8.4) and satisfies (PE) with a strict inequality; in fact,

$$
\tilde{u}_{t}+F\left(t, x, \tilde{u}, D \tilde{u}, D^{2} \tilde{u}\right) \leq-\frac{\varepsilon}{(T-t)^{2}} .
$$

Since $u \leq v$ follows from $\tilde{u} \leq v$ in the limit $\varepsilon \downarrow 0$, it will simply suffice to prove the comparison under the additional assumptions

$$
\left\{\begin{array}{l}
\text { (i) } u_{t}+F\left(t, x, u, D u, D^{2} u\right) \leq-\varepsilon / T^{2}<0 \text { and } \\
\text { (ii) } \lim _{t \uparrow T} u(t, x)=-\infty \text { uniformly on } \bar{\Omega} \text {. }
\end{array}\right.
$$

We will assume

$$
(s, z) \in(0, T) \times \Omega \text { and } u(s, z)-v(s, z)=\delta>0
$$

and then contradict this assumption. We may assume that $u,-v$ are bounded above. Let $(\hat{t}, \hat{x}, \hat{y})$ be a maximum point of $u(t, x)-v(t, y)-(\alpha / 2)|x-y|^{2}$ over $[0, T) \times \bar{\Omega} \times \bar{\Omega}$ where $\alpha>0$; such a maximum exists in view of the assumed bound above on $u,-v$, the compactness of $\bar{\Omega}$, and (8.7)(ii). The purpose of the term $(\alpha / 2)|x-y|^{2}$ is as in the elliptic case. Set

$$
M_{\alpha}=u(\hat{t}, \hat{x})-v(\hat{t}, \hat{y})-\frac{\alpha}{2}|\hat{x}-\hat{y}|^{2} .
$$

By (8.8), $M_{\alpha} \geq \delta$. If $\hat{t}=0$, we have

$$
0<\delta \leq M_{\alpha} \leq \sup _{\bar{\Omega} \times \bar{\Omega}}\left(\psi(x)-\psi(y)-\frac{\alpha}{2}|x-y|^{2}\right) ;
$$


however, the right-hand side above tends to zero as $\alpha \rightarrow \infty$ by Lemma 3.1, so $\hat{t}>0$ if $\alpha$ is large. Likewise, $\hat{x}, \hat{y} \in \Omega$ if $\alpha$ is large by $u \leq v$ on $[0, T) \times \partial \Omega$. Thus we may apply Theorem 8.3 at $(\hat{t}, \hat{x}, \hat{y})$ to learn that there are numbers $a, b$ and $X, Y \in \mathscr{S}(N)$ such that

$$
(a, \alpha(\hat{x}-\hat{y}), X) \in \overline{\mathscr{P}}_{\mathscr{O}}^{2,+} u(\hat{t}, \hat{x}), \quad(b, \alpha(\hat{x}-\hat{y}), Y) \in \overline{\mathscr{P}}_{\mathscr{O}}^{2,-} v(\hat{t}, \hat{y})
$$

such that

$$
a-b=0 \text { and }-3 \alpha\left(\begin{array}{ll}
I & 0 \\
0 & I
\end{array}\right) \leq\left(\begin{array}{cc}
X & 0 \\
0 & -Y
\end{array}\right) \leq 3 \alpha\left(\begin{array}{cc}
I & -I \\
-I & I
\end{array}\right) .
$$

The relations

$$
\begin{gathered}
a+F(\hat{t}, \hat{x}, u(\hat{t}, \hat{x}), \alpha(\hat{x}-\hat{y}), X) \leq-c, \\
b+F(\hat{t}, \hat{y}, v(\hat{t}, \hat{y}), \alpha(\hat{x}-\hat{y}), Y) \geq 0,
\end{gathered}
$$

and (8.10) imply

$$
\begin{aligned}
c & \leq F(\hat{t}, \hat{y}, v(\hat{t}, \hat{y}), \alpha(\hat{x}-\hat{y}), Y)-F(\hat{t}, \hat{x}, u(\hat{t}, \hat{x}), \alpha(\hat{x}-\hat{y}), X) \\
& \leq \omega(\alpha|\hat{x}-\hat{y}|+|\hat{x}-\hat{y}|)
\end{aligned}
$$

which leads to a contradiction as in the proof of Theorem 3.3.

Let us mention a couple of other adaptations of results above to parabolic problems. Section 5.B may be regarded as establishing continuity of solutions with respect to boundary data and the equation itself. In the parabolic case, there is the initial data, the boundary data, and the equation to consider. In the above context, we may consider a solution of $u_{t}+F\left(t, x, D u, D^{2} u\right) \leq 0$ in $(0, T) \times \Omega$ and a solution of $v_{t}+G\left(t, x, D v, D^{2} v\right) \geq 0$ in $(0, T) \times \Omega$ with the continuity and boundedness properties assumed in Theorem 8.2. Suppose $g(t) \geq(G(t, x, p, X)-F(t, x, p, X))^{+}$where $g$ is continuous and $(u(t, x)-$ $v(t, x))^{+} \leq K_{1}$ for $(t, x) \in(0, T) \times \partial \Omega,(u(0, x)-v(0, x))^{+} \leq K_{2}$ for $x \in \bar{\Omega}$. Then the function $w(x, t)=v(x, t)+\max \left(K_{1}, K_{2}\right)+\int_{0}^{t} g(s) d s$ is a solution of $w_{t}+F\left(t, x, D w, D^{2} w\right) \geq 0$. In this way, if we have comparison, we conclude that

$$
u(t, x) \leq v(t, x)+\max \left(\sup _{(0, T) \times \partial \Omega}(u-v)^{+}, \sup _{\{0\} \times \bar{\Omega}}(u-v)^{+}\right)+\int_{0}^{t} g(s) d s .
$$

In many cases we may put

$$
g(t)=\sup _{\mathbb{R}^{N} \times \mathbb{R}^{N} \times \mathscr{S}(N)}(G(t, \cdot)-F(t, \cdot))^{+},
$$

and a simple example is $G(t, x, p, X)=F(t, x, p, X)+f(t, x)$.

Notes on $\S 8$. The presentation follows M. G. Crandall and H. Ishii [48]. Let us also mention that it is well recognized that most results concerning stationary equations have straightforward parabolic analogues; indeed, the parabolic situation is often better, since

$$
u_{t}+F\left(x, u, D u, D^{2} u\right)=0
$$

more or less corresponds to $\lambda u+F\left(x, u, D u, D^{2} u\right)=0$ with large $\lambda>0$. Moreover, the special linear dependence on $u_{t}$ in the parabolic case allows one to let $F$ depend on $t$ in a merely measurable manner; see, e.g., H. Ishii [84], B. Perthame and P. L. Lions [129], N. Barron and R. Jensen [32], and 
D. Nunziante $[137,138]$. Another special feature of the parabolic case is that, owing to the special structure, one can often allow rather singular initial data. For example, infinite values may be allowed and semicontinuity may suffice, depending on the situation (see, e.g., M. G. Crandall, P. L. Lions, and P. E. Souganidis [57] and E. N. Barron and R. Jensen [33, 34]).

\section{SiNGULAR EQUATIONS: AN EXAMPLE FROM GEOMETRY}

Let $p \otimes q=\left\{p_{i} q_{j}\right\}$, the matrix with entries $p_{i} q_{j}$. It can be shown that if $\psi$ is a smooth function and $D \psi$ does not vanish on the level set $\Gamma=\{\psi=c\}$ and $u$ is a classical solution of the Cauchy problem

$$
u_{t}-\operatorname{trace}\left(\left(I-\frac{D u \otimes D u}{|D u|^{2}}\right) D^{2} u\right)=0, \quad u(0, x)=\psi(x)
$$

on some strip $(0, T) \times \mathbb{R}^{N}$, then $\Gamma_{t}=\{u(t, \cdot)=c\}$ represents the result of evolving $\Gamma$ according to its mean curvature to the time $t$, whence there is geometrical interest in (9.1). Indeed, in less regular situations where we have viscosity solutions, $\Gamma_{t}$ has been proposed as a definition of the result of evolving $\Gamma$ in this way. The nonlinearity involved, which we hereafter denote $F(p, X)=-\operatorname{trace}\left(\left(I-(p \otimes p) /|p|^{2}\right) X\right)$, is degenerate elliptic on the set $p \neq 0$ and undefined at $p=0$. Thus the results of the preceeding sections do not apply immediately. However, this is easy to remedy using the special form of the equation. The extensions of $F$ to $(0, X)$ given by

$$
\underline{F}(p, X)=\left\{\begin{array}{ll}
F(p, X) & \text { if } p \neq 0, \\
-2\|X\| & \text { if } p=0,
\end{array} \quad \bar{F}(p, X)= \begin{cases}F(p, X) & \text { if } p \neq 0 \\
2\|X\| & \text { if } p=0\end{cases}\right.
$$

are lower semicontinuous and upper semicontinuous respectively. We define $u$ to be a subsolution (respectively, supersolution) of $u_{t}+F\left(D u, D^{2} u\right)=0$ if it is a subsolution of $u_{t}+\underline{F}\left(D u, D^{2} u\right)=0$ (respectively, of $u_{t}+\bar{F}\left(D u, D^{2} u\right)=0$ ) and a solution if it is both a subsolution and a supersolution. The reason that this will succeed is roughly that, in the analysis, we will need only to insert $X=0$ when we have to deal with $p=0$, so what one does with $p=0$ is not important so long as it is consistent. To illustrate matters in a slightly simpler setting, let us consider instead the stationary problem

$$
u+F\left(D u, D^{2} u\right)-f(x)=0 \text { in } \mathbb{R}^{N},
$$

where we use the corresponding definitions of subsolutions, etc. Theorem 5.1 remains valid for the current $F$.

Theorem 9.1. Let $f \in \mathrm{UC}\left(\mathbb{R}^{N}\right)$. Then ((9.3) has a unique solution $u \in \mathrm{UC}\left(\mathbb{R}^{N}\right)$.

We sketch the proof, which proceeds according to the outline given in the proof of Theorem 5.1 with slight twists.

Proof of comparison. The comparison proof is a slight modification of that of $\S 5 . \mathrm{D}$. We begin assuming that $u, v$ are a subsolution and a supersolution of (9.3) and $u(x)-v(y) \leq L(1+|x|+|y|)$ (which is (5.11), and then proceed as before, ending up with

$$
\begin{array}{r}
u(\hat{x})-v(\hat{y}) \leq f(\hat{x})-f(\hat{y})+\bar{F}\left(\hat{p}-D \beta_{R}(\hat{y}),-\hat{Z}-D^{2} \beta_{R}(\hat{y})\right) \\
-\underline{F}\left(\hat{p}+D \beta_{R}(\hat{x}), \hat{Z}+D^{2} \beta_{R}(\hat{x})\right)
\end{array}
$$


in place of (5.14). This still implies a bound on $u(x)-v(y)-2 K|x-y|$ as before. Then proceeding still further with the proof, we adapt slightly and consider a maximum point $(\hat{x}, \hat{y})$ of

$$
\Phi(x, y)=u(x)-v(y)-\left(\alpha|x-y|^{4}+\varepsilon\left(|x|^{2}+|y|^{2}\right)\right),
$$

which will exist by virtue of the bound already obtained. We assume, without loss of generality for what follows, that $\Phi(\hat{x}, \hat{y}) \geq 0$ so that for some $C$

$$
\alpha|\hat{x}-\hat{y}|^{4}+\varepsilon\left(|\hat{x}|^{2}+|\hat{y}|^{2}\right) \leq u(\hat{x})-v(\hat{y}) \leq 2 K|\hat{x}-\hat{y}|+C .
$$

Using Remark 3.8, we then have the existence of $X, Y \in \mathscr{S}(N)$ such that

$$
(\hat{p}+2 \varepsilon \hat{x}, X+2 \varepsilon I) \in \bar{J}^{2,+} u(\hat{x}), \quad(\hat{p}-2 \varepsilon \hat{y}, Y-2 \varepsilon I) \in \bar{J}^{2,-} v(\hat{y})
$$

where

$$
\begin{aligned}
\hat{p} & =4 \alpha|\hat{x}-\hat{y}|^{2}(\hat{x}-\hat{y}), \\
\|X\|,\|Y\| & \leq C_{1} \alpha|\hat{x}-\hat{y}|^{2} \quad \text { and } \quad X \leq Y .
\end{aligned}
$$

Now (9.5) implies that $\varepsilon\left(|\hat{x}|^{2}+|\hat{y}|^{2}\right)$ and $\alpha|\hat{x}-\hat{y}|^{3}$, and hence $\hat{p}$, are bounded independently of $\varepsilon \leq 1$ for fixed $\alpha \geq 1$ while $\alpha|\hat{x}-\hat{y}|^{4}$ is also bounded independently of $\alpha$. Hence, $\varepsilon \hat{x}, \varepsilon \hat{y} \rightarrow 0$ as $\varepsilon \downarrow 0$. We have the following analogue of (5.18)

$$
u(\hat{x})-v(\hat{y}) \leq(\bar{F}(\hat{p}-2 \varepsilon \hat{y}, Y-2 \varepsilon I)-\underline{F}(\hat{p}+2 \varepsilon \hat{x}, X+2 \varepsilon I))+f(\hat{y})-f(\hat{x})
$$

that leads, by use of the estimates above, to

$$
\limsup _{\varepsilon \downarrow 0}(u(\hat{x})-v(\hat{y})) \leq \bar{F}\left(p, Y_{0}\right)-\underline{F}\left(p, X_{0}\right)+\kappa\left(C /(\alpha)^{1 / 4}\right)
$$

where $C$ is some constant and $\left(p, X_{0}, Y_{0}\right)$ is a limit point of $(\hat{p}, X, Y)$ as $\varepsilon \downarrow 0$. If $p \neq 0$, we are done since

$$
\bar{F}\left(p, Y_{0}\right)-\underline{F}\left(p, X_{0}\right)=F\left(p, Y_{0}\right)-F\left(p, X_{0}\right) \leq 0
$$

because $X_{0} \leq Y_{0}$. If $p=0$, we use the information $4 \alpha|\hat{x}-\hat{y}|^{2}(\hat{x}-\hat{y}) \rightarrow$ $p=0$ and (9.7) (recall $\alpha$ is fixed) to conclude that $X_{0}=Y_{0}=0$, and then $\bar{F}\left(p, Y_{0}\right)=\underline{F}\left(p, X_{0}\right)=0$, and we are still done.

Proof that solutions lie in $\mathrm{UC}\left(\mathbb{R}^{N}\right)$. This is exactly as in $\S 5 . \mathrm{D}$.

Proof of existence. A supersolution and subsolution are produced exactly as in $\S 5 . D$. Perron's method still applies here, since $\underline{F}$ is lower semicontinuous and, we may use Lemma 4.4 as is with $F$ replaced by $\underline{F}$ and the variant of Lemma 4.4 given in Remark 4.5 with $G_{-}=\underline{F}$ and $G^{+}=\bar{F}$.

Notes on $\S 9$. As shown in $\S 1$, a number of equations arising from geometrical considerations present singularities at $p=0$. The fact that this can easily be circumvented was shown independently by L. C. Evans and J. Spruck [72] and Y. Chen, Y. Giga, and S. Goto [45]. We also mention the work of H. M. Soner [152] on the equation (9.1) and the papers by G. Barles [16] and S. Osher and J. Sethian [140], which showed how various geometrical questions about "moving fronts" could be reduced to equations that can be handled by viscosity theory. A general class of singular equations is treated in Y. Giga, S. Goto, H. Ishii, and M. H. Sato [80] and M. H. Sato [146]; these works establish existence and uniqueness as well as convexity properties of solutions. 


\section{APPLICATIONS AND PERSPECTIVES}

In this section we list some applications of the theory of viscosity solutions and indicate some of the promising directions for development of the theory in the next few years. We give some important references but they are not exhaustive.

To begin this rather long list of applications, we recall that perhaps the main motivation for developing the theory was its relevance for the theories of Optimal Control and Differential Games. Indeed, as is well known, in the theory of optimal control of ordinary differential equations or stochastic differential equations (with complete observations) or in the theory of zero sum, two player deterministic or stochastic differential games, the Dynamic Programming Principle (DPP for short) states that the associated value functions should be characterized as the solutions of associated partial differential equations. These equations are called Bellman or or Hamilton-Jacobi-Bellman (HJB for short) equations in control theory or Isaacs equations in differential games. The DPP was, however, heuristic and proofs of it required more regularity of the value functions than they usually enjoy. The flexibility of the theory of viscosity solutions has completely filled this regularity gap: roughly speaking, value functions are viscosity solutions and are uniquely determined by this fact (via the uniqueness of viscosity solutions). See, e.g., [70, 116, 118, 119, 153]. This basic theoretical fact allows a spectacular simplification of the theory of deterministic differential games $[70,153]$ and also provided the possibility of creating sound mathematical foundations for stochastic differential games [77]. See also [88, $136]$ in addition to $[29,30]$.

The generalization of the definition of viscosity solutions to systems in diagonal form is rather straightforward and has applications to optimal control and differential games. For these topics see, e.g., [43, 63, 92, 95, 114, 147]. In the case of systems, a combination of viscosity solutions and weak solutions based on distribution theory may define a natural notion of weak solutions [80].

More generally, as usual, a better understanding of existence-uniqueness issues for classes of equations leads to a better understanding of more specific issues. Typical examples here are perturbation questions, asymptotic problems, and a more detailed solution of some specific applications to Engineering or Finance problems $[35,161,162]$. Also, the part of the theory concerned with boundary conditions has led to a rather complete theory for problems with state-constraints (at least for deterministic problems) that are enforced by cost or boundary mechanisms $[149,150,44,90,25,26,33,34]$. Let us also mention that an interesting link between viscosity solutions and the other main argument of Control Theory, the Pontryagin principle, has been shown [31, 9]. Of course, last but not least, these results have led to numerical approaches to Control or Differential Games problems via the resolution of the HJB (or Isaacs) equation.

Indeed, the viscosity solutions theory is intimately connected with numerical analysis and scientific computing. First of all, it provides efficient tools to perform convergence analyses (e.g., [52, 28, 133, 155]). It also indicates how to build discretization methods or schemes for other general boundary conditions and in particular for classical boundary conditions when working with rather degenerate equations [73, 144].

Another consequence of efficient existence, uniqueness, approximation, and 
convergence results is the possibility of establishing or discovering various qualitative properties of solutions (formulae, representations, singularities, geometrical properties, characterizations and properties of semigroups, ...-[12, 40, $41,54,56,70,88,124,131])$. Of course, one of the most important qualitative properties is the regularity of solutions. Viscosity solutions, because of their flexibility and their pointwise definition, have led to regularity results that are spectacular either in their generality (regularizing effects, Lipschitz regularity, or semicontinuity- $[122,116,57,20,18,17])$ or by their originality $\left(C^{1, \alpha}, C^{2, \alpha}\right.$ or pointwise $L^{p}$ estimates- $\left.[39,96,158,159,160,103]\right)$.

The uniqueness and convergence parts of the theory have made possible partial differential equations approaches to various asymptotic problems like large deviations $[68,76,11,141]$, geometrical optics $[71,23,22,13,69]$, or homogenization problems $[66,123,128]$ by arguments that are both powerful and simple.

More specific applications concern the interplay between the behavior of solutions at infinity and structure conditions $[83,54,56,134]$ or the treatment of integrodifferential operators $[112,148]$. Other applications concern some particular classes of equations arising in Engineering like some models of the propagation of fronts in Combustion Theory [16, 140], or the so-called shapes from shading models in Vision Theory [144].

Finally, a large part of the theory (but not yet all of it) has been "raised" to infinite-dimensional equations both for first-order and second-order equations [55, 125-127]. In addition to "standard" extensions to infinite-dimensional spaces, specific applications like the optimal control of partial differential equations or even stochastic partial differential equations-this last topic being motivated in particular by the optimal control of the so-called Zakai's operation, a well-known formulation of optimal control problems of finite-dimensional diffusion processing with partial observations-require some new developments of the viscosity solutions theory in order to accommodate unbounded terms in the equations [55, Parts IV and V; 126, 8, 7, 151, 42, 157].

The above rather vague and general comments on applications already contain many hints concerning promising directions the theory of viscosity solutions may take in the near future. In particular, the infinite-dimensional part of the theory will most probably explode in view of the unbounded possible avenues of investigation. Much more progress is also to be expected for degenerate second-order equations and boundary conditions. In particular, progress is to be made on existence questions for Dirichlet (and state constraints) boundary conditions - this might have a considerable impact on various applications like models in Finance. Similarly, we expect progress on uniqueness and regularity questions for uniformly elliptic second-order equations.

It is also reasonable to hope that the insight gained by viscosity solutions will help to devise efficient high-order schemes for numerical approximations and prove their convergence.

More specific developments should (and will) concern questions of behavior at infinity, geometrical optics problems, the use and the theory of discontinuous solutions, and the investigation of "second-order" integrodifferential operators

Of course, the reader should not restrict his imagination to the borders we drew above. 
APPENDIX. The PROOF OF THEOREM 3.2

In this section we sketch the proof of Theorem 3.2, which we reproduce here for convenience.

Theorem 3.2. Let $\mathscr{O}_{i}$ be a locally compact subset of $R^{N_{i}}$ for $i=1, \ldots, k$,

$$
\mathscr{O}=\mathscr{O}_{1} \times \cdots \times \mathscr{O}_{k},
$$

$u_{i} \in \operatorname{USC}\left(\mathscr{O}_{i}\right)$, and $\varphi$ be twice continuously differentiable in a neighborhood of O. Set

$$
w(x)=u_{1}\left(x_{1}\right)+\cdots+u_{k}\left(x_{k}\right) \text { for } x=\left(x_{1}, \cdots, x_{k}\right) \in \mathscr{O},
$$

and suppose $\hat{x}=\left(\hat{x}_{1}, \ldots, \hat{x}_{k}\right) \in \mathscr{O}$ is a local maximum of $w-\varphi$ relative to $\mathscr{O}$. Then for each $\varepsilon>0$ there exists $X_{i} \in \mathscr{S}\left(N_{i}\right)$ such that

$$
\left(D_{x_{i}} \varphi(\hat{x}), X_{i}\right) \in \bar{J}_{\mathscr{O}}^{2,+} u_{i}\left(\hat{x}_{i}\right) \text { for } i=1, \ldots, k
$$

and the block diagonal matrix with entries $X_{i}$ satisfies

$$
-\left(\frac{1}{\varepsilon}+\|A\|\right) I \leq\left(\begin{array}{ccc}
X_{1} & \ldots & 0 \\
\vdots & \ddots & \vdots \\
0 & \ldots & X_{k}
\end{array}\right) \leq A+\varepsilon A^{2}
$$

where $A=D^{2} \varphi(\hat{x}) \in \mathscr{S}(N), N=N_{1}+\cdots+N_{k}$.

The sketch we give below will be somewhat abbreviated, but the main points are all displayed and the reader should be able to provide any omitted details from the indications given.

As defined and used in the main text, $\operatorname{USC}(\mathscr{O})$ consisted of the upper semicontinuous functions mapping $\mathscr{O}$ into $\mathbb{R}$; however, at this point it is convenient to allow the value " $-\infty$," so we hereafter set

$$
\operatorname{USC}(\mathscr{O})=\{\text { upper semicontinuous functions } u: \mathscr{O} \rightarrow \mathbb{R} \cup\{-\infty\}\} .
$$

The convenience is well illustrated by the following reduction. Below, conditions written for the index $i$ are understood to hold for $i=1, \ldots, k$.

First Reduction. We may as well assume that $\mathscr{O}_{i}=\mathbb{R}^{N_{i}}$. Indeed, if not, we first restrict $u_{i}$ to a compact neighborhood $K_{i}$ of $\hat{x}_{i}$ in $\mathscr{O}_{i}$ and then extend the restriction to $\mathbb{R}^{N_{i}}$ by $u_{i}\left(x_{i}\right)=-\infty$ if $x_{i} \notin K_{i}$ (abusing notation and still writing $\left.u_{i}\right)$. The compactness of $K_{i}$ guarantees that $u_{i} \in \operatorname{USC}\left(\mathbb{R}^{N_{i}}\right)$; one then checks that $\bar{J}_{\mathscr{\theta}}^{2,+} u_{i}\left(\hat{x}_{i}\right)=\bar{J}^{2,+} u_{i}\left(\hat{x}_{i}\right)$ (provided $-\infty<u_{i}\left(\hat{x}_{i}\right)$ ). It is clear that $\hat{x}$ is still a local maximum of $w-\varphi$ relative to $\mathbb{R}^{N}=\mathbb{R}^{N_{1}+\cdots+N_{k}}$. This device of putting functions equal to $-\infty$ on unimportant sets will be used several times below to localize considerations to any compact neighborhood of a point under consideration.

Second Reduction. We may as well assume that $\hat{x}=0, u_{i}(0)=0, \varphi(x)=$ $\frac{1}{2}\langle A x, x\rangle$ for some $A \in \mathscr{S}(N)$ is a pure quadratic, and 0 is a global maximum of $w-\varphi$. Indeed, a translation puts $\hat{x}$ at the origin and then by replacing $\varphi(x), u_{i}\left(x_{i}\right)$ by $\varphi(x)-(\varphi(0)+\langle D \varphi(0), x\rangle)$ and $u_{i}\left(x_{i}\right)-\left(u_{i}(0)+\left\langle D_{x_{i}} \varphi(0), x_{i}\right\rangle\right)$, we reduce to the situation $\hat{x}=D \varphi(0)=0$, and $\varphi(0)=u_{i}(0)=0$. Then, since 
$\varphi(x)=\frac{1}{2}\langle A x, x\rangle+o\left(|x|^{2}\right)$ where $A=D^{2} \varphi(0)$ and $w(x)-\varphi(x) \leq w(0)-\varphi(0)=$ 0 for small $x$, if $\eta>0$ we will have $w(x)-\frac{1}{2}\langle(A+\eta I) x, x\rangle<0$ for small $x \neq 0$. Globality of the (strict) maximum at 0 may be achieved by localizing via the first reduction. If the result holds in this case, we may use the bounds asserted in Theorem 3.2 to pass to the limit as $\eta \downarrow 0$ to obtain the full result.

Thus we have to prove the following

Theorem 3.2'. Let $u_{i} \in \operatorname{USC}\left(\mathbb{R}^{N_{i}}\right), u_{i}(0)=0$, for $i=1,2, \ldots, k, N=$ $N_{1}+\cdots+N_{k}, A \in \mathscr{S}(N)$, and

(A.2) $w(x)=u_{1}\left(x_{1}\right)+\cdots+u_{k}\left(x_{k}\right) \leq \frac{1}{2}\langle A x, x\rangle$ for $x=\left(x_{1}, \cdots, x_{k}\right) \in \mathbb{R}^{N}$.

Then for each $\varepsilon>0$, there exists $X_{i} \in \mathscr{S}\left(N_{i}\right)$ such that

$$
\left(0, X_{i}\right) \in \bar{J}_{\mathscr{\theta}}^{2,+} u_{i}\left(\hat{x}_{i}\right) \text { for } i=1, \ldots, k,
$$

and the block diagonal matrix with entries $X_{i}$ satisfies

$$
-\left(\frac{1}{\varepsilon}+\|A\|\right) I \leq\left(\begin{array}{ccc}
X_{1} & \ldots & 0 \\
\vdots & \ddots & \vdots \\
0 & \ldots & X_{k}
\end{array}\right) \leq A+\varepsilon A^{2} .
$$

Here are the main steps in the proof:

Step 1. Introduction of the sup convolutions. If $\varepsilon>0$, the Cauchy-Schwarz inequality yields

$$
\langle A x, x\rangle \leq\left\langle\left(A+\varepsilon A^{2}\right) \xi, \xi\right\rangle+\left(\frac{1}{\varepsilon}+\|A\|\right)|x-\xi|^{2} \text { for }, x, \xi \in \mathbb{R}^{N} .
$$

Putting

$$
\lambda=\frac{1}{\varepsilon}+\|A\|
$$

and using (A.2) and (A.5) we find

(A.6)

$$
\left(u_{1}\left(x_{1}\right)-\frac{\lambda}{2}\left|x_{1}-\xi_{1}\right|^{2}\right)+\cdots+\left(u_{k}\left(x_{k}\right)-\frac{\lambda}{2}\left|x_{k}-\xi_{k}\right|^{2}\right) \leq \frac{1}{2}\left\langle\left(A+\varepsilon A^{2}\right) \xi, \xi\right\rangle
$$

or $w(x)-(\lambda / 2)|x-\xi|^{2} \leq(1 / 2)\left\langle\left(A+\varepsilon A^{2}\right) \xi, \xi\right\rangle$. If necessary, we modify the definition of the $u_{i}$ off a neighborhood of 0 to assure that they are bounded above. Put

$$
\text { (A.7) } \hat{w}(\xi)=\sup _{x \in \mathbb{R}^{N}}\left(w(x)-\frac{\lambda}{2}|x-\xi|^{2}\right), \hat{u}_{i}\left(\xi_{i}\right)=\sup _{x_{i} \in \mathbb{R}^{N_{i}}}\left(u_{i}\left(x_{i}\right)-\frac{\lambda}{2}\left|x_{i}-\xi_{i}\right|^{2}\right)
$$

so that $\hat{w}(\xi)=\hat{u}_{1}\left(\xi_{1}\right)+\cdots+\hat{u}_{k}\left(\xi_{k}\right)$. Observe that, choosing $x_{i}=0, \hat{u}_{i}(0) \geq$ $u_{i}(0)=0$ while $\hat{u}_{1}(0)+\cdots+\hat{u}_{k}(0) \leq 0$, so $\hat{u}_{i}(0)=0$.

Step 2. Two results on semiconvex functions: Aleksandrov's Theorem and Jensen's Lemma. The functions $\hat{w}$ and $\hat{u}_{i}$ are semiconvex; more precisely, since the supremum of convex functions is convex, $\hat{w}(\xi)+(\lambda / 2)|\xi|^{2}$ and $\hat{u}_{i}(\xi)+$ $(\lambda / 2)\left|\xi_{i}\right|^{2}$ are convex. We then call $\lambda$ a semiconvexity constant for $\hat{w}$ and $\hat{u}_{i}$. This is the reason we will employ two nontrivial facts about semiconvex functions. The first assertion is a classical result of Aleksandrov:

Theorem A.2. Let $\varphi: \mathbb{R}^{N} \rightarrow \mathbb{R}$ be semiconvex. Then $\varphi$ is twice differentiable almost everywhere on $\mathbb{R}^{N}$. 
Proof. Since we assume that the members of our audience who are not functional analytically oriented have already seen a proof of this result, we will give one for those who are so oriented. In the course of proof we assume certain facts about convex and Lipschitz functions that are known to most readers. References will be given in the notes.

We may as well assume that $\varphi$ is convex; a convex function on $\mathbb{R}^{N}$ is locally Lipschitz and hence once differentiable almost everywhere. We let

$$
F_{1}=\left\{x \in \mathbb{R}^{N}: D \varphi(x) \text { exists }\right\} ;
$$

the letter $F$ being used to indicate that $F_{1}$ has full measure (i.e., its complement is a null set). The subdifferential $\partial \varphi$ of $\varphi$ is the set-valued function given by

$$
\hat{y} \in \partial \varphi(\hat{x}) \quad \text { if } \varphi(x) \geq \varphi(\hat{x})+\langle\hat{y}, x-\hat{x}\rangle \quad \text { for } x \in \mathbb{R}^{N} .
$$

It is clear that $\partial \varphi(x)=\{D \varphi(x)\}$ whenever $\varphi$ is differentiable at $x$. In particular, $\partial \varphi$ is single valued almost everywhere. By convex analysis, $J=(I+\partial \varphi)^{-1}$ is a single-valued mapping of $\mathbb{R}^{N}$ into itself and is nonexpansive (has Lipschitz constant 1). (Given $x \in \mathbb{R}^{N}$, the solution $z$ of $z+\partial \varphi(z) \ni x$ is the value of $J$ at $x ; z$ is the minimum of $q \rightarrow \varphi(q)+\frac{1}{2}|x-q|^{2}$.) Since $\varphi$ is defined on all of $\mathbb{R}^{N}, \partial \varphi(z)$ is nonempty for every $z$. We claim that for almost all $x \in \mathbb{R}^{N}$ $D \varphi$ is differentiable at $x$, i.e., there exists $A \in \mathscr{S}(N)$ such that

$$
D \varphi(y)=D \varphi(x)+A(y-x)+o(|y-x|) \text { for } y \in F_{1}
$$

for almost all $x \in \mathbb{R}^{N}$.

To establish this, let

$$
F_{2}=\{J(x): J \text { is differentiable at } x \text { and } D J(x) \text { is nonsingular }\} .
$$

Since $J$ is Lipschitz continuous and onto $\mathbb{R}^{N}$ (because $\partial \varphi(z)$ is nonempty for every $z), F_{2}$ has full measure. We use here the facts that $\{x: D J(x)$ exists $\}$ is a set of full measure, $J$ maps null sets to null sets and $J(\{x: D J(x)$ exists and is singular $\}$ ) is null. It is to be shown that $D \varphi$ is differentiable on $F_{3}=F_{1} \cap F_{2}$, which is a set of full measure. By the definition of $J$,

$$
D \varphi(J(x))=x-J(x) \text { for } J(x) \in F_{3} \subset F_{1} .
$$

Thus assume $J(x)+\delta y \in F_{1}$ where $\delta y$ is small. By assumption, $D \varphi(J(x)+\delta y)$ exists. Since $J$ is Lipschitz and $D J(x)$ is nonsingular, if $\delta y$ is sufficiently small, there is a $\delta x$ solving $J(x+\delta x)=J(x)+\delta y$, and we may choose $\delta x$ to satisfy $|\delta x| \leq K|\delta y|$ for some constant $K$. Since $J$ is a contraction, we also have $|\delta y| \leq|\delta x|$ and thus $|\delta x|$ and $|\delta y|$ are comparable. Then

$$
\begin{aligned}
D \varphi(J(x)+\delta y) & =D \varphi(J(x+\delta x))=x+\delta x-J(x+\delta x) \\
& =D \varphi(J x)+(\delta x-D J(x) \delta x)+o(\delta x) \\
& =D \varphi(J x)+(I-D J(x)) \delta x+o(\delta y) .
\end{aligned}
$$

It remains to see that $\delta x=(D J(x))^{-1} \delta y+o(\delta y)$; however, this follows from the fact that $|\delta x|$ and $|\delta y|$ are comparable and the relation $J(x)+D J(x) \delta x+$ $o(\delta x)=J(x)+\delta y$. We conclude that $D(D \varphi)(J(x))$ exists and is $D J(x)^{-1}-I$.

It remains to establish that

$$
\varphi(J(x)+\delta y)=\varphi(J(x))+D \varphi(J(x)) \delta y+\frac{1}{2}\left\langle\left(D J(x)^{-1}-I\right) \delta y, \delta y\right\rangle+o\left(|\delta y|^{2}\right)
$$


for $J(x) \in F_{3}$. However, if we let $\psi(\delta y)=\varphi(J(x)+\delta y)$ and $\tilde{\psi}(\delta y)=$ $\varphi(J(x))+D \varphi(J x) \delta y+\frac{1}{2}\left\langle\left(D J(x)^{-1}-I\right) \delta y, \delta y\right\rangle$, we have $\psi(0)=\tilde{\psi}(0)$ and for almost all small $\delta y$

$$
\begin{aligned}
D \psi(\delta y) & =D \varphi(J(x)+\delta y)=D \varphi(J(x))+(D J(x))^{-1} \delta y-\delta y+o(\delta y) \\
& =D \tilde{\psi}(\delta y)+o(\delta y)
\end{aligned}
$$

thus $\Psi(\delta y)=\psi(\delta y)-\tilde{\psi}(\delta y)$ is locally Lipschitz continuous and satisfies $\Psi(0)=$ 0 and $D \Psi(\delta y)=o(\delta y)$ for almost all small $\delta y$. It is clear that then $\Psi(\delta y)=$ $o\left(|\delta y|^{2}\right)$, whence the result.

The next result we will need concerning semiconvex functions, which we call Jensen's lemma, follows. In the statement, $B(x, r)$ is the closed ball of radius $r$ centered at $x$ and $B_{r}$ is the ball centered at the origin.

Lemma A.3. Let $\varphi: \mathbb{R}^{N} \rightarrow \mathbb{R}$ be semiconvex and $\hat{x}$ be a strict local maximum point of $\varphi$. For $p \in \mathbb{R}^{N}$, set $\varphi_{p}(x)=\varphi(x)+\langle p, x\rangle$. Then for $r, \delta>0$, $K=\left\{x \in B(\hat{x}, r)\right.$ : there exists $p \in B_{\delta}$ for which $\varphi_{p}$ has a local maximum at $\left.x\right\}$ has positive measure.

Proof. We assume that $r$ is so small that $\varphi$ has $\hat{x}$ as a unique maximum point in $B(\hat{x}, r)$ and assume for the moment that $\varphi$ is $C^{2}$. It follows from this that if $\delta$ is sufficiently small and $p \in B_{\delta}$, then every maximum of $\varphi_{p}$ with respect to $B(\hat{x}, r)$ lies in the interior of $B(\hat{x}, r)$. Since $D \varphi+p=0$ holds at maximum points of $\varphi_{p}, D \varphi(K) \supset B_{\delta}$. Let $\lambda \geq 0$ and $\varphi(x)+(\lambda / 2)|x|^{2}$ be convex; we then have $-\lambda I \leq D^{2} \varphi$; moreover, on $K, D^{2} \varphi \leq 0$ and then

$$
-\lambda I \leq D^{2} \varphi(x) \leq 0 \text { for } x \in K .
$$

In particular, $\left|\operatorname{det} D^{2} \varphi(x)\right| \leq \lambda^{N}$ for $x \in K$. Thus

$$
\operatorname{meas}\left(B_{\delta}\right) \leq \operatorname{meas}(D \varphi(K)) \leq \int_{K}\left|\operatorname{det} D^{2} \varphi(x)\right| d x \leq \operatorname{meas}(K)|\lambda|^{N}
$$

(see the notes) and we have a lower bound on the measure of $K$ depending only on $\lambda$.

In the general case, in which $\varphi$ need not be smooth, we approximate it via mollification with smooth functions $\varphi_{\varepsilon}$ that have the same semiconvexity constant $\lambda$ and that converge uniformly to $\varphi$ on $B(\hat{x}, r)$. The corresponding sets $K_{\varepsilon}$ obey the above estimates for small $\varepsilon$ and

$$
K \supset \bigcap_{n=1}^{\infty} \bigcup_{m=n}^{\infty} K_{1 / m}
$$

is evident. The result now follows.

Step 3. A consequence of Step 2 and magic properties of sup convolution. Lemma A. 4 below applies to $\hat{w}$ of Step 1 and we shall see it provides us with matrices $X_{i} \in \mathscr{S}\left(N_{i}\right)$ such that $\left(0, X_{i}\right) \in \bar{J}^{2,+} \hat{u}_{i}(0)$ and (A.3) holds.

We use the notation

$$
J^{2} f(z)=J^{2,+} f(z) \cap J^{2,-} f(z)
$$

from which one defines $\bar{J}^{2}$ analogously to $\bar{J}^{2,+}, \bar{J}^{2,-}$. Note that $(p, X) \in$ $J^{2} f(x)$ amounts to

$$
f(y)=f(x)+\langle p, y-x\rangle+\frac{1}{2}\langle X(y-x), y-x\rangle+o\left(|x-y|^{2}\right) \text { as } y \rightarrow x
$$

i.e., $f$ is twice differentiable at $x$ and $p=D f(x), X=D^{2} f(x)$. 
Lemma A.4. If $f \in C\left(\mathbb{R}^{N}\right), B \in \mathscr{S}(N), f(\xi)+(\lambda / 2)|\xi|^{2}$ is convex and $\max _{\mathbb{R}^{N}}\left(f(\xi)-\frac{1}{2}\langle B \xi, \xi\rangle\right)=f(0)$, then there is an $X \in \mathscr{S}(N)$ such that $(0, X) \in$ $\bar{J}^{2} f(0)$ and $-\lambda I \leq X \leq B$.

Proof. Clearly $f(\xi)-\frac{1}{2}\langle B \xi, \xi\rangle-|\xi|^{4}$ has a strict maximum at $\xi=0$. By Aleksandrov's Theorem and the convexity assumption, $f$ is twice differentiable a.e. and then by Jensen's Lemma, for every $\delta>0$ there exists $q_{\delta} \in \mathbb{R}^{N}$ with $\left|q_{\delta}\right| \leq \delta$ such that $f(\xi)+\left\langle q_{\delta}, \xi\right\rangle-\frac{1}{2}\langle B \xi, \xi\rangle-|\xi|^{4}$ has a maximum at a point $\xi_{\delta}$ with $\left|\xi_{\delta}\right| \leq \delta$ and $f(\xi)$ is twice differentiable at $\xi_{\delta}$. From calculus, $\left|q_{\delta}\right|,\left|\xi_{\delta}\right| \leq \delta$, and the convexity of $f(\xi)+(\lambda / 2)|\xi|^{2}$,

$$
D f\left(\xi_{\delta}\right)=O(\delta), \quad-\lambda I \leq D^{2} f\left(\xi_{\delta}\right) \leq B+O\left(\delta^{2}\right) .
$$

Observing that $\left(D f\left(\xi_{\delta}\right), D^{2} f\left(\xi_{\delta}\right)\right) \in J^{2} f\left(x_{\delta}\right)$, we conclude the proof upon passing to a subsequential limit (selected so that $D^{2} f\left(\xi_{\delta}\right)$ is convergent) as $\delta \downarrow 0$.

Applying Lemma A.4 to $\hat{w}$ from (A.7) with $B=A+\varepsilon A^{2}$ and noting that the values of $J^{2} \hat{w}$ are formed as one expects given the representation $\hat{w}=$ $\hat{u}_{1}+\cdots+\hat{u}_{k}$, we obtain $\left(0, X_{i}\right) \in \bar{J}^{2} \hat{u}_{i}(0), i=1, \ldots, k$ such that (A.3) holds. The final step in the proof of Theorem $3.2^{\prime}$ is provided by applying the next lemma to each $u_{i}$ to conclude that $\left(0, X_{i}\right) \in \bar{J}^{2,+} u_{i}(0)$.

Here are magical properties of the sup convolution.

Lemma A.5. Let $\lambda>0, v \in \operatorname{USC}\left(\mathbb{R}^{M}\right)$ bounded above, and

$$
\hat{v}(\xi)=\sup _{x \in \mathbb{R}^{M}}\left(v(x)-(\lambda / 2)|x-\xi|^{2}\right) .
$$

If $\eta, q \in \mathbb{R}^{M}, Y \in \mathscr{S}(M)$, and $(q, Y) \in J^{2,+} \hat{v}(\eta)$, then

$$
(q, Y) \in J^{2,+} v(\eta+q / \lambda) \text { and } \hat{v}(\eta)+(1 / 2 \lambda)|q|^{2}=v(\eta+q / \lambda) \text {. }
$$

In consequence, if $(0, Y) \in \bar{J}^{2,+} \hat{v}(0)$, then $(0, Y) \in \bar{J}^{2,+} v(0)$.

Proof. We assume $(q, Y) \in J^{2,+} \hat{v}(\eta)$, and let $y \in \mathbb{R}^{N}$ be a point such that $\hat{v}(\eta)=v(y)-(\lambda / 2)|y-\eta|^{2}$. Then for any $x, \xi \in \mathbb{R}^{N}$

$$
\begin{aligned}
v(x)-\frac{\lambda}{2}|\xi-x|^{2} \leq & \hat{v}(\xi) \\
\leq & \hat{v}(\eta)+\langle q, \xi-\eta\rangle \\
& +\frac{1}{2}\langle Y(\xi-\eta), \xi-\eta\rangle+o\left(|\xi-\eta|^{2}\right) \\
= & v(y)-\frac{\lambda}{2}|y-\eta|^{2}+\langle q, \xi-\eta\rangle \\
& +\frac{1}{2}\langle Y(\xi-\eta) \xi-\eta\rangle+o\left(|\xi-\eta|^{2}\right) \\
= & v(y)-\frac{\lambda}{2}|y-\eta|^{2}+\langle q, \xi-\eta\rangle+O\left(|\xi-\eta|^{2}\right) .
\end{aligned}
$$

Putting $\xi=x-y+\eta$ in the appropriate one of these relations yields

$$
v(x) \leq v(y)+\langle q, x-y\rangle+\frac{1}{2}\langle Y(x-y), x-y\rangle+o\left(|x-y|^{2}\right),
$$


while substituting $x=y$ and $\xi=\eta+\alpha(\lambda(\eta-y)+q)$ yields

$$
0 \leq \alpha|\lambda(\eta-y)+q|^{2}+O\left(\alpha^{2}\right) .
$$

The former simply says that $(q, Y) \in J^{2,+} v(y)$ while the latter with small $\alpha<0$ implies that $\lambda(\eta-y)+q=0$ or $y=\eta+q / \lambda$ as claimed. The relation $\hat{v}(\eta)+(1 / 2 \lambda)|q|^{2}=v(\eta+q / \lambda)$ follows at once. Assume now that $\left(q_{n}, Y_{n}\right) \in$ $J^{2,+} \hat{v}\left(\xi_{n}\right)$ and $\left(\xi_{n}, \hat{v}(\xi), q_{n}, Y_{n}\right) \rightarrow(0,0,0, Y)$; by the foregoing, $\left(q_{n}, Y_{n}\right) \in$ $J^{2,+} v\left(\xi_{n}+q_{n} / \lambda\right)$ and $v\left(\xi_{n}+q_{n} / \lambda\right)=\hat{v}\left(\xi_{n}\right)+(1 / 2 \lambda)\left|q_{n}\right|^{2}$. By the definitions, $v(0) \leq \hat{v}(0)$ and from this, the upper semicontinuity and the foregoing, we have

$$
v(0) \geq \limsup _{n \rightarrow \infty} v\left(\xi_{n}+\frac{q_{n}}{\lambda}\right)=\limsup _{n \rightarrow \infty}\left(\hat{v}\left(\xi_{n}\right)+\frac{1}{2 \lambda}\left|q_{n}\right|^{2}\right)=\hat{v}(0) \geq v(0),
$$

which provides the final piece of information we needed to conclude that $(0, Y)$ $\in \bar{J}^{2,+} v(0)$.

Remark A.6. We close with a final and more elegant reformulation of what was proved above: if the $u_{i}$ satisfy the conditions of Theorem 3.2, $w(x)=$ $u_{1}\left(x_{1}\right)+\cdots+u_{k}\left(x_{k}\right)$ and $\left(\left(p_{1}, \ldots, p_{k}\right), A\right) \in \bar{J}_{\Omega}^{2,+} w(\hat{x})$, then for each $\varepsilon>0$, there exists $X_{i} \in \mathscr{S}\left(N_{i}\right)$ such that $\left(p_{i}, X_{i}\right) \in \bar{J}_{\mathscr{O}}^{2,+} u_{i}\left(\hat{x}_{i}\right)$ and (A.1) holds.

Notes on the appendix. Above, we provided (for the first time) a self-contained proof of Theorem 3.2. Except for the two auxiliary results on semiconvex functions, the main tool is the so-called sup convolution. This approximation procedure (more often in the guise of inf convolution) is well known in functional analysis and, in particular, in convex analysis and the theory of maximal monotone operators (see, for example, the text [38] of H. Brezis). It was noticed in [110] that it may provide an efficient regularization procedure for (even degenerate) elliptic equations; some of its properties are given there. See also [104]. Its "magical properties" can be seen as related to the Lax formula for the solution of

$$
\frac{\partial w}{\partial t}-\frac{1}{2}|\nabla w|^{2}=0 \text { for } x \in \mathbb{R}^{N}, t \geq 0,\left.w\right|_{t=0}=v \text { on } \mathbb{R}^{N},
$$

which is

$$
w(x, t)=\sup _{y}\left\{v(y)-\frac{1}{2 t}|x-y|^{2}\right\} .
$$

Indeed, the coincidence of this solution formula and solutions produced by the method of charteristics leads to the properties used. Of course, this is a heuristic connection, since characteristic methods require too much regularity to be rigorous here.

The inf convolution can also be seen as a nonlinear analogue of the standard mollification when replacing the "linear structure of $L^{2}$ and its duality" by the "nonlinear structure of $L^{\infty}$ or $C$." One can also interpret this analogy in terms of the so-called exotic algebra $(\mathbb{R}, \max ,+)$.

Theorem A.2 is a classical result of A. D. Aleksandrov [1]. The proof given here is in fact a slightly stronger form of this result ( $D \varphi$ is differentiable a.e.) and our proof follows F. Mignot [135] (although we have made his proof more complicated for pedagogical reasons). In the proof we used the fact that Lipschitz functions are differentiable a.e., which is called Rademacher's Theorem. A 
proof may be found in L. C. Evans and R. Gariepy [67] and F. Mignot [135]. We also used that Lipschitz functions map null sets to null sets and the solvability of $J(x+\delta y)=J(x)+\delta x$ when $D J(x)$ exists and is nonsingular. These are proved in, respectively, Lemma 7.25 and the proof of Theorem 7.24 in W. Rudin [145]; see also [135]. We also used the fact that $J(\{x: D J(x)$ exists and is singular $\})$ is null. This follows from the general formula

$$
\int_{\mathbb{R}^{N}} \#\left(A \cap J^{-1}(y)\right) d y=\int_{A}|\operatorname{det} D J(x)| d x
$$

where \# is counting measure. This formula holds for Lipschitz continuous functions and measurable sets $A \subset \mathbb{R}^{N}$ and is a special case of the Area Formula for Lipschitzian maps between Euclidean spaces. The Area Formula may be found in L. C. Evans and R. Gariepy [67] and H. Federer [74].

Jensen's Lemma (Lemma A.3) is a variation on an aspect of the theme known as "Aleksandrov's maximum principle" (see $[2,3,6,37,120,143])$.

\section{REFERENCES}

1. A. D. Aleksandrov, Almost everywhere existence of the second differential of a convex function and some properties of convex functions, Leningrad. Univ. Ann. (Math. Ser.) 37 (1939), 335. (Russian)

2. __ Uniqueness conditions and estimates for the solution of the Dirichlet problem, Amer. Math. Soc. Transl., vol. 68, Amer. Math. Soc., Providence, RI, 1968, pp. 89-119.

3. __ Majorization of solutions of second-order linear equations, Amer. Math. Soc. Transl., vol. 68, Amer. Math. Soc., Providence, RI, 1968, pp. 120-143.

4. R. Alziary de Roquefort, Jeux de poursuite et approximation des fonctions valeur, Thèse, Universitè de Paris-Dauphine, 1990; Modél. Math. Anal. Num. 25 (1991), 535-560.

5. S. Aizawa and Y. Tomita, On unbounded viscosity solutions of a semilinear second order elliptic equation, Funkcial. Ekvac. 31 (1988), 141-160.

6. I. Ja. Bakelman, On the theory of quasilinear elliptic equations, Sibirsk. Mat. Z. 2 (1961), 179-186.

7. V. Barbu, Hamilton-Jacobi equations and nonlinear control problems, preprint.

8. The dynamic programming equation for the time optimal control problem in infinite dimensions, SIAM J. Control Optim. 29 (1991), 445-456.

9. V. Barbu, E. N. Barron, and R. Jensen, Necessary conditions for optimal control in Hilbert spaces, J. Math. Anal. Appl. 133 (1988), 151-162.

10. V. Barbu and G. Da Prato, Hamilton-Jacobi equations in Hilbert spaces, Research Notes in Math., vol. 86, Pitman, Boston, MA, 1983.

11. M. Bardi, An asymptotic formula for the Green's function of an elliptic operator, Ann. Scuola Norm. Sup. Pisa Cl. Sci. (4) 4 (1987), 569-612.

12. M. Bardi and L. C. Evans, On Hopf's formulas for solutions of Hamilton-Jacobi equations, Nonlinear Anal. Theory Methods Appl. 8 (1984), 1373-1381.

13. M. Bardi and B. Perthame Exponential decay to stable states in phase transitions via a double log-transformation, Comm. Partial Differential Equations 15 (1990), 1649-1669.

14. G. Barles, Existence results for first-order Hamilton-Jacobi equations, Ann. Inst. H. Poincaré Anal. Non Linéaire 1 (1984), 325-340.

15. __ Remarques sur des résultats d'existence pour les équations de Hamilton-Jacobi du premier ordre, Ann. I. H. Poincaré Anal. Non Linéaire 2 (1985), 21-33.

16. __ Remark on a flame propogation model, Rapport INRIA, no. 464, 1985.

17. Interior gradient bounds for the mean curvature equation by viscosity solution methods, Differential Integral Equations 4 (1991), 263-275. 
18. __ A weak Bernstein method for fully nonlinear elliptic equations, Differential Integral Equations 4 (1991), 241-262.

19. __ Uniqueness and regularity results for first-order Hamilton-Jacobi equations, Indiana Univ. Math. J. 39 (1990), 443-466.

20. __, Regularity results for first-order Hamilton-Jacobi equations, Differential Integral Equations 3 (1990), 103-125.

21. _ Fully nonlinear Neumann type boundary conditions for second-order elliptic and parabolic equations, J. Differential Equations (to appear).

22. G. Barles, L. Bronsard, and P. E. Souganidis, Front propagation for reaction-diffusion equations of bistable type, Analyse Non Linéaire (to appear).

23. G. Barles, L. C. Evans, and P. E. Souganidis, Wave front propagation for reaction-diffusion systems of PDE's, Duke Univ. Math. J. 61 (1990), 835-858.

24. G. Barles and P. L. Lions, Fully nonlinear Neumann type boundary conditions for first-order Hamilton-Jacobi equations, Nonlinear Anal. Theory Methods Appl. 16 (1991), 143-153.

25. G. Barles and B. Perthame, Discontinuous solutions of deterministic optimal stopping-time problems, Modèl. Math. Anal. Num. 21 (1987), 557-579.

26. __ Exist time problems in optimal control and the vanishing viscosity method, SIAM J. Control Optim. 26 (1988), 1133-1148.

27. _ Comparison principle for Dirichlet-type H. J. equations and singular perturbations of degenerate elliptic equations, Appl. Math. Optim. 21 (1990), 21-44.

28. G. Barles and P. E. Souganidis, Convergence of approximation schemes for fully nonlinear second order equations, Asymp. Anal. 4 (1991), 271-283.

29. E. N. Barron, Differential games with maximum cost, Nonlinear Anal. Theory Methods Appl. 14 (1990), 971-989.

30. E. N. Barron and H. Ishii, The Bellman equation for minimizing the maximum cost, Nonlinear Anal. Theory Methods Appl. 13 (1989), 1067-1090.

31. E. N. Barron and R. Jensen, The Pontryagin maximum principle from dynamic programming and viscosity solutions to first-order partial differential equations, Trans. Amer. Math. Soc. 298 (1986), 635-641.

32. __ Generalized viscosity solutions for Hamilton-Jacobi equations with time-measurable Hamiltonians, J. Differential Equations 68 (1987), 10-21.

33. __ Semicontinuous viscosity solutions for Hamilton-Jacobi equations with convex Hamiltonians, Comm. Partial Differential Equations 15 (1990), 1713-1742.

34. __ Optimal control and semicontinuous viscosity solutions, Proc. Amer. Math. Soc. 113 (1991), 397-402.

35. _ A stochastic control approach to the pricing of options, Math. Operations Research 15 (1990), 49-79.

36. S. Benton, The Hamilton-Jacobi equation: A global approach, Academic Press, New York, 1977.

37. A. D. Bony, Principe du maximum dans les espaces de Sobolev, C. R. Acad. Sci. Paris Ser. A 265 (1967), 333-336.

38. H. Brezis, Operateurs Maximaux Monotones et semigroupes de contractions dans les espaces de Hilbert, North Holland, Amsterdam, 1973.

39. L. Caffarelli, Interior a priori estimates for solutions of fully non-linear equations, Ann. of Math. (2) 130 (1989), 180-213.

40. P. Cannarsa and M. Soner, On the singularities of the viscosity solutions to Hamilton-JacobiBellman equations, Indiana Univ. Math. J. 46 (1987), 501-524.

41. __, Generalized one-sided estimates for solutions of Hamilton-Jacobi equations and applications, Nonlinear Anal. Theory Methods Appl. 13 (1989), 305-323.

42. P. Cannarsa, F. Gozzi, and M. Soner, A boundary value problem for Hamilton-Jacobi equations in Hilbert spaces, Appl. Math. Opt. 24 (1991), 197-220.

43. I. Cappuzzo-Dolcetta and L. C. Evans, Optimal switching for ordinary differential equations, SIAM J. Optim. Control 22 (1988), 1133-1148. 
44. I. Cappuzzo-Dolcetta and P. L. Lions, Viscosity solutions of Hamilton-Jacobi-equations and state constraints, Trans. Amer. Math. Soc. 318 (1990), 643-683.

45. Y. G. Chen, Y. Giga, and S. Goto, Uniqueness and existence of viscosity solutions of generalized mean curvature flow equations, J. Differential Geom. 33 (1991), 749-786.

46. M. G. Crandall, Quadratic forms, semidifferentials and viscosity solutions of fully nonlinear elliptic equations, Ann. I. H. Poincaré Anal. Non Linéaire 6 (1989), 419-435.

47. M. G. Crandall, L. C. Evans, and P. L. Lions, Some properties of viscosity solutions of Hamilton-Jacobi equations, Trans. Amer. Math. Soc. 282 (1984), 487-502.

48. M. G. Crandall and H. Ishii, The maximum principle for semicontinuous functions, Differential Integral Equations 3 (1990), 1001-1014.

49. M. G. Crandall, H. Ishii, and P. L. Lions, Uniqueness of viscosity solutions of HamiltonJacobi equations revisited, J. Math. Soc. Japan 39 (1987), 581-596.

50. M. G. Crandall and P. L. Lions, Condition d'unicité pour les solutions generalisées des équations de Hamilton-Jacobi du premier ordre, C. R. Acad. Sci. Paris Sér. I Math. 292 (1981), 183-186.

51. _ Viscosity solutions of Hamilton-Jacobi equations, Trans. Amer. Math. Soc. 277 (1983), $1-42$.

52. _ Two approximations of solutions of Hamilton-Jacobi equations, Math. Comp. 43 (1984), 1-19.

53. $\ldots$, On existence and uniqueness of solutions of Hamilton-Jacobi equations, Nonlin. Anal. Theory Methods Appl. 10 (1986), 353-370.

54. __ Unbounded viscosity solutions of Hamilton-Jacobi equations, Illinois J. Math. 31 (1987), 665-688.

55. __ Hamilton-Jacobi equations in infinite dimensions, Part I. Uniqueness of viscosity solutions, J. Func. Anal. 62 (1985), 379-396; Part II. Existence of viscosity solutions 65 (1986), 368-405; Part III 68 (1986), 214-247; Part IV. Unbounded linear terms 90 (1990), 237-283; Part V. B-continuous solutions, 97 (1991), 417-465.

56. _ Q Quadratic growth of solutions of fully nonlinear second order equations on $\mathbb{R}^{n}$, Differential Integral Equations 3 (1990), 601-616.

57. M. G. Crandall, P. L. Lions, and P. E. Souganidis, Maximal solutions and universal bounds for some quasilinear evolution equations of parabolic type, Arch. Rat. Mech. Anal. 105 (1989), 163-190.

58. M. G. Crandall and R. Newcomb, Viscosity solutions of Hamilton-Jacobi equations at the boundary, Proc. Amer. Math. Soc. 94 (1985), 283-290.

59. M. G. Crandall, R. Newcomb, and Y. Tomita, Existence and uniqueness of viscosity solutions of degenerate quasilinear elliptic equations in $\mathbb{R}^{n}$, Appl. Anal. 34 (1989), 1-23.

60. P. Dupuis and H. Ishii, On oblique derivative problems for fully nonlinear second order elliptic equations on nonsmooth domains, Nonlinear Anal. Theory Methods Appl. 12 (1991), 1123-1138.

61. On oblique derivative problems for fully nonlinear second order elliptic PDE's on domains with corners, Hokkaido Math. J. 20 (1991), 135-164.

62. P. Dupuis, H. Ishii, and H. M. Soner, $A$ viscosity solution approach to the asymptotic analysis of queuing systems, Ann. Probab. 18 (1990), 226-255.

63. H. Engler and S. Lenhart, Viscosity solutions for weakly coupled systems of Hamilton-Jacobi equations, Proc. London Math. Soc. 63 (1991), 212-240.

64. L. C. Evans, A convergence theorem for solutions of nonlinear second order elliptic equations, Indiana Univ. Math. J. 27 (1978), 875-887.

65. __ On solving certain nonlinear differential equations by accretive operator methods, Israel J. Math. 36 (1980), 225-247.

66. __ The perturbed test function technique for viscosity solutions of partial differential equations, Proc. Roy. Soc. Edinburgh Sect. A 111 (1989), 359-375.

67. L. C. Evans and R. Gariepy, Measure theory and fine properties of functions, Studies in Advanced Math., CRC Press, Ann Arbor, 1992. 
68. L. C. Evans and H. Ishii, A pde approach to some asymptotic problems concerning random differential equations with small noise intensities, Ann. I. H. Poincaré Anal. Non Linéaire 2 (1985), 1-20.

69. L. C. Evans, M. Soner, and P. E. Souganidis, The Allen-Cahn equation and generalized motion by mean curvature, preprint.

70. L. C. Evans and P. E. Souganidis, Differential games and representation formulas for solutions of Hamilton-Jacobi-Isaacs equations, Indiana Univ. Math. J. 33 (1984), 773-797.

71. _ A PDE approach to geometric optics for semilinear parabolic equations, Indiana Univ. Math. J. 38 (1989), 141-172.

72. L. C. Evans and J. Spruck, Motion of level sets by mean curvature. I, J. Differential Geom. 33 (1991), 635-681.

73. M. Falcone, A numerical approach to the infinite horizon problem of deterministic control theory, Appl. Math. Optim. 15 (1987), 1-13.

74. H. Federer, Geometric measure theory, Springer, New York, 1969.

75. W. H. Fleming and R. Rishel, Determinisitic and stochastic optimal control, Springer-Verlag, New York, 1975.

76. W. H. Fleming and P. E. Souganidis, PDE-viscosity solution approach to some problems of large deviations, Ann. Scuola Norm. Sup. Pisa Cl. Sci. (4) 13 (1986), 171-192.

77. __, On the existence of value functions of two player, zero-sum stochastic differential games, Indiana Univ. Math. J. 38 (1989), 293-314.

78. H. Frankowska, On the single-valuedness of Hamilton-Jacobi operators, Nonlin. Anal. Theory Methods Appl. 10 (1986), 1477-1483.

79. Y. Giga, S. Goto, H. Ishii, and M.-H. Sato, Comparison principle and convexity preserving properties for singular degenerate parabolic equations on unbounded domains, Indiana $\mathrm{U}$. Math. J. 40 (1991), 443-470.

80. Y. Giga, S. Goto, and H. Ishii, Global existence of weak solutions for interface equations coupled with diffusion equations, SIAM J. Math. Anal. (to appear).

81. D. Gilbarg and N. S. Trudinger, Elliptic partial differential equations of second order, 2nd Ed., Springer-Verlag, New York, 1983.

82. H. Ishii, Remarks on existence and uniqueness of viscosity solutions of Hamilton-Jacobi equations, Bull. Fac. Sci. Eng. Chuo Univ. 26 (1983), 5-24.

83. __ Uniqueness of unbounded viscosity solutions of Hamilton-Jacobi equations, Indiana Univ. Math. J. 33 (1984), 721-748.

84. _ Hamilton-Jacobi equations with discontinuous Hamiltonians on arbitrary open sets, Bull. Fac. Sci. Eng. Chuo Univ. 28 (1985), 33-77.

85. __ Existence and uniqueness of solutions of Hamilton-Jacobi equations, Funkcial. Ekvac. 29 (1986), 167-188.

86. __ Perron's method for Hamilton-Jacobi equations, Duke Math. J. 55 (1987), 369-384.

87. __ A simple direct proof for uniqueness for solutions of the Hamilton-Jacobi equations of eikonal type, Proc. Amer. Math. Soc 100 (1987), 247-251.

88. _ Representation of solutions of Hamilton-Jacobi equations, Nonlin. Anal. Theory Methods Appl. 12 (1988), 121-146.

89. __, On uniqueness and existence of viscosity solutions of fully nonlinear second-order elliptic PDE's, Comm. Pure Appl. Math. 42 (1989), 14-45.

90. __ A boundary value problem of the Dirichlet type for Hamilton-Jacobi equations, Ann. Scuola Norm. Sup. Pisa Cl. Sci. (4) 16 (1989), 105-135.

91. __ Fully nonlinear oblique derivative problems for nonlinear second-order elliptic PDE's, Duke Math. J. 62 (1991), 663-661.

92. __ Perron's method for monotone systems of second-order elliptic PDEs, Diff. Integral Equations 5 (1992), 1-24.

93. H. Ishii and S. Koike, Remarks on elliptic singular perturbation problems, Appl. Math. Optim. 23 (1991), 1-15. 
94. _ _ Viscosity solutions of a system of nonlinear second-order elliptic PDEs arising in switching games, Funkcial. Ekvac. 34 (1991), 143-155.

95. __ Viscosity solutions for monotone systems of second-order elliptic PDE's, Comm. Partial Differential Equations 16 (1991), 1095-1128.

96. H. Ishii and P. L. Lions, Viscosity solutions of fully nonlinear second-order elliptic partial differential equations, J. Differential Equations 83 (1990), 26-78.

97. H. Ishii and N. Yamada, A remark on a system of inequalities with bilateral obstacles, Nonlinear Anal. Theory Methods Appl. 13 (1989), 1295-1301.

98. A. V. Ivanov, Quasilinear degenerate and nonuniformly elliptic equations of second order, Proc. Steklov Inst. Math. 1 (1984), 1-287.

99. S. Koike, On the rate of convergence of solutions in singular perturbation problems, J. Math. Anal. Appl. 157 (1991), 277-292.

100. __ An asymptotic formula for solutions of Hamilton-Jacobi-Bellman equations, Nonlinear Anal. Theory Methods Appl. 11 (1987), 429-436.

101. R. Jensen, The maximum principle for viscosity solutions of fully nonlinear second order partial differential equations, Arch. Rat. Mech. Anal. 101 (1988), 1-27.

102. __ Uniqueness criteria for viscosity solutions of fully nonlinear elliptic partial differential equations, Indiana Univ. Math. J. 38 (1989), 629-667.

103. __ Local quadratic growth estimates for elliptic obstacle problems, preprint.

104. R. Jensen, P. L. Lions, and P. E. Souganidis, A uniqueness result for viscosity solutions of second order fully nonlinear partial differential equations, Proc. Amer. Math. Soc. 102 (1988), 975-978.

105. R. Jensen and P. E. Souganidis, regularity result for viscosity solutions of Hamilton-Jacobi equations in one space dimension, Trans. Amer. Math. Soc. 301 (1987), 137-147.

106. J. J. Kohn and L. Nirenberg, Degenerate elliptic-parabolic equations of second order, Comm. Pure Appl. Math. 20 (1967), 797-872.

107. S. N. Kruzkov, First order quasilinear equations in several independent variables, Math. USSR-Sb. 10 (1970), 217-243.

108. N. V. Krylov, Controlled diffusion processes, Springer-Verlag, Berlin, 1980.

109. __ Nonlinear elliptic and parabolic equations of second order, D. Reidel Publishing Co., Boston, MA, 1987.

110. J. M. Lasry and P. L. Lions, A remark on regularization in Hilbert spaces, Israel J. Math 55 (1986), 257-266.

111. __ Nonlinear elliptic equations with singular boundary conditions and stochastic control with state constraints, I, The model problem, Math. Ann. 283 (1989), 583-630.

112. S. Lenhart, Integro-differential operators associated with diffusion processes with jumps, Appl. Math. Optim. 9 (1982), 177-191.

113. __ Viscosity solutions for weakly coupled systems of first order PDEs, J. Math. Anal. Appl. 131 (1988), 180-193.

114. S. Lenhart and N. Yamada, Perron's method for viscosity solutions associated with piecewisedeterministic processes, Funkcial. Ekvac. (to appear).

115. __ Viscosity solutions associated with switching game for piecewise-deterministic processes, preprint.

116. P. L. Lions, Generalized solutions of Hamilton-Jacobi equations, Research Notes in Mathematics 69, Pitman, Boston, MA, 1982.

117. __ On the Hamilton-Jacobi-Bellman equations, Acta Appl. 1 (1983), 17-41.

118. _ Some recent results in the optimal control of diffusion processes: Stochastic analysis, Proceedings of the Taniguchi International Symposium on Stochastic Analysis (Katata and Kyoto 1982) Kinokuniya, Tokyo, 1984.

119. __ Optimal control of diffusion processes and Hamilton-Jacobi-Bellman equations. Part 1 : The dynamic programming principle and applications and Part 2: Viscosity solutions and uniqueness, Comm. Partial Differential Equations 8 (1983), 1101-1174 and 1229-1276. 
120. $\_$A remark on the Bony maximum principle, Proc. Amer. Math. Soc. 88 (1983), 503508.

121. Neumann type boundary conditions for Hamilton-Jacobi equations, Duke J. Math. 52 (1985), 793-820.

122. $\ldots$, Regularizing effects for first-order Hamilton-Jacobi equations, Applic. Anal. 20 (1985), 283-308.

123. __ Equations de Hamilton-Jacobi et solutions de viscositè, Colloque De Giorgi, Paris, 1983; Pitman, London, 1985.

124. __ Some properties of the viscosity semigroups of Hamilton-Jacobi equations, Nonlinear Differential Equations and Applications, Pitman, London, 1988.

125. _ _ Viscosity solutions of fully nonlinear second-order equations and optimal stochastic control in infinite dimensions. Part I : The case of bounded stochastic evolutions, Acta Math. 161 (1988), 243-278.

126. _ _ Viscosity solutions of fully nonlinear second-order equations and optimal stochastic control in infinite dimensions. Part II: Optimal Control of Zakai's Equation, Stochastic Partial Differential Equations and Applications II, Lecture Notes in Math., vol. 1390, Springer, Berlin, 1989. (G. DuPrato and L. Tubaro, eds.) Proceedings of the International Conference on Infinite Dimensional Stochastic Differential Equations, Trento, Springer, Berlin, 1988.

127. _ _ Viscosity solutions of fully nonlinear second-order equations and optimal stochastic control in infinite dimensions. Part III : Uniqueness of viscosity solutions of general second order equations, J. Funct. Anal. 86 (1989), 1-18.

128. P. L. Lions, G. Papanicolau, and S. R. S. Varadhan, Homogenization of Hamilton-Jacobi equations, preprint.

129. P. L. Lions and B. Perthame, Remarks on Hamilton-Jacobi-Equations with measurable timedependent Hamiltonians, Nonlinear Anal. Theory Methods Appl. 11 (1987), 613-622.

130. P. L. Lions and M. Nisio, $A$ uniqueness result for the semigroup associated with the HamiltonJacobi-Bellman operator, Proc. Japan Acad. 58 (1982), 273-276.

131. P. L. Lions and Rochet, Hopf formula and multitime Hamilton-Jacobi equations, Proc. Amer. Math. Soc. 90 (1980), 79-84.

132. P. L. Lions and P. E. Souganidis, Viscosity solutions of second-order equations, stochastic control and stochastic differential games, Stochastic Differential Systems, Stochastic Control Theory and Applications (W. H. Fleming and P. L. Lions, eds.), IMA Vol. Math. Appl., vol. 10, Springer, Berlin, 1988.

133. _ Convergence of MUSCL type methods for scalar conservation laws, C. R. Acad. Sci. Paris Sér. A 311 (1990), 259-264.

134. P. L. Lions, P. E. Souganidis, and J. Vazquez, The relation between the porous medium equation and the eikonal equation in several space dimensions, Rev. Math. Ibero. 3 (1987), 275-310.

135. F. Mignot, Contrôle optimal dans les inéquations variationelles elliptiques, J. Funct. Anal. 22 (1976), 130-185.

136. R. Newcomb, Existence and correspondence of value functions and viscosity solutions of Hamilton-Jacobi equations Univ. of Wisconsin-Madison, 1988.

137. D. Nunziante, Uniqueness of viscosity solutions of fully nonlinear second-order fully nonlinear parabolic equations with discontinuous time-dependence, Differential Integral Equations 3 (1990), 77-91.

138. $\ldots$ Existence and uniqueness of unbounded viscosity solutions of parabolic equations with discontinuous time-dependence, preprint.

139. O. A. Oleinnik and E. V. Radekvic, Second order equations with nonnegative characteristic form, American Mathematical Society, Providence, RI, 1973.

140. S. Osher and J. Sethian, Fronts propagating with curvature dependent speed: algorithms based on Hamilton-Jacobi formulations, J. Comp. Physics 79 (1988), 12-49.

141. B. Perthame, Perturbed dynamical systems with an attracting singularity and weak viscosity limits in H. J. equations, Trans. Amer. Math. Soc. 317 (1990), 723-747. 
142. B. Perthame and R. Sanders, The Neumann problem for fully nonlinear second order singular perturbation problems, SIAM J. Math. Anal. 19 (1988), 295-311.

143. C. Pucci, Su una limitazone per soluzioni di equazioni ellittche, Boll. Un. Mat. Ital. (6) 21 (1966), 228-233.

144. E. Rouy and A. Tourin, $A$ viscosity solution approach to shape-from shading, preprint.

145. W. Rudin, Real and complex analysis, 3rd ed., Mc Graw-Hill, New York, 1987.

146. M. Sato, Comparison principle for singular degenerate elliptic equations on unbounded domains, Proc. Japan Acad. Ser. A 66 (1990), 252-256.

147. N. Yamada, Viscosity solutions for a system of inequalities with biblateral obstacles, Funkcial. Ekvac. 30 (1987), 417-425.

148. A. Sayah, Equations d'Hamilton-Jacobi du premier ordre avec termes intégro differentiels. Partres I and II, Comm. Partial Differential Equations 16 (1991), 1057-1221.

149. M. Soner, Optimal control with state-space constraint I, SIAM J. Control Optim. 24 (1986), 552-562.

150. __ Optimal control with state-space constraint II, SIAM J. Control Optim. 24 (1986), 1110-1122.

151. __, On the Hamilton-Jacobi-Bellman equations in Banach spaces, J. Optim. Theory Appl. 57 (1988), 121-141.

152. __ Motion of a set by the curvature of its mean boundary, preprint.

153. P. E. Souganidis, Max-Min representations and product formulas for the viscosity solutions of Hamilton-Jacobi equations with applications to differential games, Nonlin. Anal. Theory Methods Appl. 9 (1985), 217-257.

154. __ Existence of viscosity solutions of Hamilton-Jacobi equations, J. Differential Equations 56 (1985), 345-390.

155. __ Approximation schemes for viscosity solutions of Hamilton-Jacobi equations, J. Differential Equations 59 (1985), 1-43.

156. __ A remark about viscosity solutions at the boundary, Proc. Amer. Math. Soc. 96 (1986), 323-330.

157. D. Tataru, Viscosity solutions of Hamilton-Jacobi equations with unbounded nonlinear terms, J. Math. Anal. Appl. 163 (1992), 345-392.

158. N. S. Trudinger, Comparison principles and pointwise estimates for viscosity solutions of second order elliptic equations, Rev. Mat. Ibero. 4 (1988), 453-468.

159. __ Hölder gradient estimates for fully nonlinear elliptic equations, Proc. Roy. Soc. Edinburgh Sect. A 108 (1988), 57-65.

160. _ On the twice differentiability of viscosity solutions of nonlinear elliptic equations, Bull. Austral. Math. Soc. 39 (1989), 443-447.

161. J. L. Vila and T. Zariphopolou, Optimal consumption and portfolio choice with borrowing constraints, preprint.

162. T. Zariphopolou, Investment-consumption model with transaction costs and Markov-Chain parameters, SIAM J. Control Optim. 30 (to appear, 1992).

Department of Mathematics, University of California, Santa Barbara, California 93106

Department of Mathematics, Chuo University, Bunkyo-Ku, Tokyo 112, Japan

Ceremade, Université Paris-Dauphine, Place de lattre de Tassigny, 75775 Paris Cedex 16, France 